\title{
Studies on safety issues in anticoagulant management
}

Citation for published version (APA):

Eijgenraam, P. (2015). Studies on safety issues in anticoagulant management. [Doctoral Thesis, Maastricht University]. Uitgeverij BOXPress. https://doi.org/10.26481/dis.20151217pe

Document status and date:

Published: 01/01/2015

DOI:

10.26481/dis.20151217pe

Document Version:

Publisher's PDF, also known as Version of record

\section{Please check the document version of this publication:}

- A submitted manuscript is the version of the article upon submission and before peer-review. There can be important differences between the submitted version and the official published version of record.

People interested in the research are advised to contact the author for the final version of the publication, or visit the DOI to the publisher's website.

- The final author version and the galley proof are versions of the publication after peer review.

- The final published version features the final layout of the paper including the volume, issue and page numbers.

Link to publication

\footnotetext{
General rights rights.

- You may freely distribute the URL identifying the publication in the public portal. please follow below link for the End User Agreement:

www.umlib.nl/taverne-license

Take down policy

If you believe that this document breaches copyright please contact us at:

repository@maastrichtuniversity.nl

providing details and we will investigate your claim.
}

Copyright and moral rights for the publications made accessible in the public portal are retained by the authors and/or other copyright owners and it is a condition of accessing publications that users recognise and abide by the legal requirements associated with these

- Users may download and print one copy of any publication from the public portal for the purpose of private study or research.

- You may not further distribute the material or use it for any profit-making activity or commercial gain

If the publication is distributed under the terms of Article $25 \mathrm{fa}$ of the Dutch Copyright Act, indicated by the "Taverne" license above, 
Studies on safety issues in

\section{anticoagulant management}


(C) Pieter Eijgenraam, Maastricht 2015

Cover: Boris Eijgenraam

Layout: Tiny Wouters

Printed by: Proefschriftmaken.nl || Uitgeverij BOXPress

ISBN: 978-90-9029390-5 


\title{
Studies on safety issues in
}

\section{anticoagulant management}

\author{
PROEFSCHRIFT \\ Ter verkrijging van de graad van doctor aan de Universiteit Maastricht, \\ op gezag van de Rector Magnificus, Prof. Dr. L.L.G. Soete, \\ volgens het besluit van het College van Decanen, \\ in het openbaar te verdedigen op \\ donderdag 17 december 2015 om 10.45 uur \\ door
}

\section{Pieter Eijgenraam}

Geboren op 27 oktober 1963 te Leiden 


\section{Promotor}

Prof. dr. H. ten Cate

\section{Copromotores}

Dr. A.J. ten Cate-Hoek

Dr. R. van den Ham (Philips Research)

\section{Beoordelingscommissie :}

Prof. dr. J.G. Maessen (voorzitter)

Dr. J.F.B.M. Fiolet

Prof. dr. P.W. Kamphuisen (UMC Groningen)

Prof. dr. R.P. Koopmans

Dr. M.J.H.A. Kruip (Erasmus Medisch Centrum Rotterdam) 


\section{Contents}

$\begin{array}{lll}\text { Chapter } 1 \quad \text { General Introduction } & 7\end{array}$

Chapter 2 The effect of clinical decision support on adherence to 19 thrombosis prophylaxis guidelines in medical patients; a single center experience

Chapter 3 Safety and efficacy of bridging with low molecular weight 35 heparins: a systematic review and partial meta-analysis

Chapter 4 Practice of bridging anticoagulation; guideline adherence and 57 risk factors for bleeding

Chapter 5 Effects of peri-operative bridging with low molecular weight heparins on coagulation during interruption of vitamin $\mathrm{K}$ antagonists: a mechanistic study

Chapter 6 Venous stenting after deep venous thrombosis and antithrombotic therapy: a systematic review

Chapter 7 Quality of anticoagulant therapy and in-stent thrombosis in patients with venous stents

Chapter 8 General discussion

Samenvatting

Valorisatie

List of publications

Dankwoord 



\section{Chapter 1}

General introduction 
Chapter 1 


\section{Hemostasis}

Blood coagulation is responsible for the prevention of fatal blood loss but also for the occurrence of venous and arterial thrombi in the vascular system, leading to a partial or total interruption of the blood stream. Primary or secondary prevention of venous or arterial thromboembolism (TE) often encompasses the administration of oral and/or parenteral anticoagulant agents. All anticoagulant use increases the risk of bleeding. Bleedings attributable to anticoagulant use can sometimes be fatal. The determination of the ideal type, dosing and timing of anticoagulant therapy is an ongoing challenge for patient care; a delicate balance has to be found between the risk of thromboembolism and bleeding for all individual patients.

The coagulation system is based on well balanced steps regulated by the coagulation proteases, the vessel wall and platelets. The liver is responsible for the production of most of the coagulation proteases like factors V, VII, IX, X, XI, XII, prothrombin and fibrinogen. The coagulation system can be activated in two ways, via the intrinsic and the extrinsic pathway. Thrombin is the central enzyme in blood coagulation, the product of a series of protease directed protein cleavages, starting with the exposure of tissue factor (TF) on the sub-endothelium or at microparticles in the circulation. The tenase and prothrombinase complex jointly further catalyze the transformation of prothrombin into thrombin and result in a burst of thrombin. ${ }^{1}$

Different mechanisms lead to the inhibition of coagulation. In one of these mechanisms thrombin in complex with thrombomodulin enables the activation of protein $C$ into activated protein $C$ (APC). APC associated with protein $S$ in turn inactivates activated FV (FVa) and FVIIla, leading to inhibition of thrombin production via a negative feedback loop. ${ }^{1}$

\section{Thrombosis and bleeding}

Abnormalities in coagulation can lead to the formation of a thrombus. Arterial TE, a major health problem especially in the elderly population, is associated with conditions such as atrial fibrillation, arteriosclerosis, or the introduction of mechanical aortic/mitral valves prosthesis. Deep venous thrombosis (DVT) and subsequently pulmonary embolism (PE), collectively referred to as venous thromboembolism (VTE) is caused by diverse risk conditions including malignant neoplasms, hospital or nursing home confinement, trauma/surgery and neurological disease with extremity paresis. The primary and secondary prevention of potentially life threatening and invalidating TE requires adequate antithrombotic treatment, which consists mainly of 
anticoagulant and/or antiplatelet therapy. ${ }^{2}$ The most important adverse effect of anticoagulant treatment is the risk of bleeding. Ideally, when antithrombotic therapy is applied a balance is found between the risk of TE and bleeding.

\section{Anticoagulant agents}

In day to day practice a wide range of anticoagulants is available for the prevention of $\mathrm{TE}^{3}$ In this thesis we discuss safety and efficacy aspects of vitamin K antagonists (VKA) and low molecular weight heparins (LMWH) in different settings. VKA inhibits the activity of the vitamin $\mathrm{K}$ dependent procoagulant proteins prothrombin, FVII, FIX and FX, and the anticoagulant proteins $C$ and S. VKA inhibits the process of recycling vitamin $\mathrm{K}$ by blocking VKORC1, resulting in a relative vitamin $\mathrm{K}$ deficiency in the liver cell. This results in the production of impaired, non $Y$ carboxylated coagulation factors. ${ }^{4,5}$ The anticoagulant effect of LMWH is mainly derived from the anti FXa effect induced by a conformational change of antithrombin (AT). In the presence of LMWH the anticoagulant effect of AT, particularly against FXa, is accelerated. ${ }^{5}$

\section{Anticoagulation assays}

Several blood tests are developed to monitor the anticoagulation intensity in patients. The most commonly used test to monitor VKA therapy is the prothrombin time (PT). ${ }^{4}$ This test responds to changes in concentration of $\mathrm{FII}, \mathrm{VII}$ and $\mathrm{X}$, which are reduced by acenocoumarol and fenprocoumon, VKAs commonly used in the Netherlands. This reduction is proportional to the half-life of the clotting factor; the first days after the (re) initiation of VKA therapy PT mainly reflects the changes in F VII, which has a relatively short half-life of 6 hours. The PT assay is performed by adding calcium and a thromboplastin to citrated plasma and is expressed in seconds. Due to for instance different sensitivity of thromboplastins used the PT initially lacked standardization. In 1982 a calibration model was adapted to standardize reporting by correcting for different thromboplastins used in different laboratories; International Normalized Ratio (INR) = (patient PT/mean normal PT) ${ }^{\text {ISI }}$ where International Sensitivity Index (ISI) denotes the thromboplastin used in the local laboratory. ${ }^{6}$ Currently, the intensity of VKA is measured by PT and expressed as INR. Frequent measurement of an individual's INR value is required in order to manage anticoagulation within a therapeutic range (internationally, an INR range of 2.0-3.0 is most common; in this country 2.5-3.5 is the most relevant range). 
The anti-Xa assay is designed to monitor the effect of LMWH and unfractionated heparin (UFH). There is no recommendation in guidelines for repeated use of an antiXa assay for dose adjustment in patients using $\mathrm{LMWH}$ for prophylaxis or treatment options. According to experts in the field, the anticoagulant effect of LMWH only needs to be monitored in obese patients, patients with reduced renal clearance ${ }^{7,8}$ and during pregnancy. ${ }^{9}$ Although there is little firm evidence for appropriate therapeutic anti-Xa ranges (also considering the fact that there is little evidence supporting a strong correlation between anti-Xa activity and efficacy) the therapeutic peak (2-4 hours after subcutaneous injection) window is estimated at 1-2 units/ml for odd LMWH and 0.5-1.0 units/ml for bid dosed LMWH. Renal impairment and multiple therapeutic doses of LMWH can result in bioaccumulation and therefore increased risk of bleeding. Testing anti-Xa level in such patients would seem appropriate.

The thrombin generation (TG) assay offers a global view on hemostasis. This assay can be performed in both platelet poor and platelet rich plasma. TG measures the concentration of thrombin over time formed after triggering coagulation with one of the recommended stimuli: $1 \mathrm{pM}$ TF, $1 \mathrm{pM}$ TF + thrombomodulin, and $5 \mathrm{pM}$ TF. TG results in a curve which describes the variation of the amount of thrombin during the activation of coagulation cascade in time. ${ }^{10}$ The 2 most important parameters obtained from this curve are peak height, the maximum concentration of thrombin at a certain moment in time and the endogenous thrombin potential which represents the total amount of thrombin produced over time. ${ }^{10}$ The position of TG measurement in practice has not been established yet. Eventually, it is likely that simplified versions of TG, for instance based on the whole blood point of care prototype assay ${ }^{11}$, will allow broad applications, including for predicting TE and bleeding, or to monitor anticoagulant therapy with VKA, LMWH, UFH and NOAC. ${ }^{10}$

\section{Thrombosis prophylaxis and CDS}

Thrombosis prophylaxis is a major topic, since many hospitalized patients are at increased risk for VTE, due to for instance increased age, immobility, cancer or surgery. The yearly incidence of DVT in the Netherlands is 0.6-1.2 cases per 1000 inhabitants, ${ }^{12}$ the reported VTE incidence in hospitalized patients is 100 times greater. ${ }^{13}$ In case of low VTE risk only early ambulation is advised; for patients at increased risk, apart from early ambulation, daily doses of LMWH is the preferred treatment option. Solid evidence of the efficacy of VTE prevention is presented in numerous studies. ${ }^{14-16}$ However, large studies show that antithrombotic prophylaxis is underused in the hospital setting, leading to avoidable cases of VTE. In different 
studies only as little as $30-50 \%$ of the patients received appropriate prophylactic therapy. ${ }^{17-19}$ In medical patients the rates of patients receiving appropriate prophylaxis are even lower than in surgical patients. ${ }^{20}$ Reasons for underutilization include unfamiliarity or disagreement with current guidelines, underestimation of VTE risk or fear for bleeding complications.

Over the last years the use of clinical decision support (CDS) has gained more attention. Prospectively validated risk assessment models (RAM) for VTE risk factors are available for integration in CDS; risk factors for VTE and bleeding are awarded with a score; simply adding the numbers after establishing a cut-off point, results in an advice to whether or not apply prophylactic interventions and in which dose. These prophylactics include pharmacological interventions (LMWH, fondaparinux or UFH) or mechanical interventions (graduated compression stockings, intermittent pneumatic compression). Available RAMs for bleeding risk estimation are not prospectively validated yet. ${ }^{15}$ High scores for VTE risk factors and low bleeding scores result in the advice to apply pharmacological interventions, a high VTE risk score combined with a high bleeding score results in the advice to apply mechanical measures and in case of a low VTE and bleeding score only the advice of early ambulation is given. Several studies in different settings have been performed to evaluate the effectiveness of different CDS systems. In most studies a positive effect of CDS was established, translating in increased guideline adherence or even a reduction of the incidence of VTE. $^{21,22}$ In some studies the effectiveness of CDS was temporary. Studies evaluating CDS systems in which a direct link to the ordering system was provided and/or CDS use was mandatory showed the best results. ${ }^{21,23,24}$

In chapter $\mathbf{2}$ of this thesis we present results of a study evaluating a pilot CDS system (from September $1^{\text {st }}$ to December $1^{\text {st }}$ 2013) in the Maastricht University Medical Center+ (MUMC+). CDS was introduced in cooperation with Philips Group Innovation Research, Eindhoven, the Netherlands, on two different wards comprising mainly of medical patients. The ACCP guidelines 2012, the prevention of VTE in nonsurgical patients, ${ }^{15}$ were used to build the CDS. The application was installed on 4 stand-alone personal computers in two different wards. The hospital patient data system (SAP, Germany) menu was extended with a dedicated CDS button on the opening page of the patient's record. A direct link to the ordering system of pharmacological or mechanical prophylaxis was not provided. All attending physicians of the wards were trained in the use of the CDS system and motivated to use CDS daily for all admitted patients. 


\section{Bridging therapy}

Anticoagulation in patients receiving long-term VKA undergoing surgery is challenging in terms of maintaining the delicate balance between bleeding and TE; the patient using anticoagulants is vulnerable for bleeding in the perioperative period. During a preferably short periprocedural period the patient should not be anticoagulated, to avoid bleeding during and after the intervention. The anticoagulation-free interval is minimized by "bridging" with LMWH or UFH which replace the longer acting VKA. The efficacy and safety of bridging with LMWH has however not been unequivocally established. ${ }^{3}$ In chapter $\mathbf{3}$ of this thesis results are presented of a systematic review analyzing the safety and efficacy aspects of bridging therapy. Several studies show that the risk of bleeding during bridging therapy is increased, while TE risk is unknown. ${ }^{25-32}$ Furthermore, guidelines for optimal use of bridging anticoagulation seem poorly adhered to. ${ }^{33}$ In chapter 4 results of a study analyzing guideline adherence in a Dutch University hospital are presented.

In 2012 the American College of Chest Physicians (ACCP) presented their latest guidelines for perioperative management of antithrombotic therapy. ${ }^{34}$ In the management of perioperative anticoagulation in patients on VKA, 3 options can be considered. First, in procedures with a low bleeding risk and the possibility of local hemostatic measures, such as dental extractions, cataract operations and small dermatologic procedures, it is considered a safe choice to continue VKA use. Second, in patients at low risk of TE, ACCP guidelines recommend stopping warfarin administration 5 days before the intervention and restarting warfarin 12-24 hours after the procedure when adequate hemostasis is secured. Finally, in patients at moderate or high TE risk current ACCP guidelines recommend bridging therapy consisting of parenteral administration of LMWH or UFH in the periprocedural period, combined with interruption of VKA use. The decision to apply bridging anticoagulation should always be based on an assessment of individual patient- and surgery-related factors. ${ }^{34}$ The suggested TE risk stratification by the ACCP is based mainly on indirect evidence from studies outside of the perioperative setting involving patients with a mechanical heart valve, chronic atrial fibrillation or VTE who either were not receiving anticoagulation or were receiving less-effective treatment. ${ }^{34-37}$ It is conceivable that due to the lack of insight in hypo or hypercoagulability during bridging therapy in individual patients the concept of bridging anticoagulation might be qualified as a 'black box'. In chapter $\mathbf{5}$ we present the results of a study in which the (interactive) effects of the co-administration of VKA and LMWH and surgery on different coagulation assays, including thrombin generation assay, INR, anti Xa assays and the 
measurement of concentrations of vitamin $\mathrm{K}$ dependent coagulation factors, were assessed.

\section{Venous stenting}

Post thrombotic syndrome (PTS) develops in more than $50 \%$ of patients with iliofemoral DVT (IFDVT). ${ }^{38}$ Conventional treatment regimes, comprising of a combination of compression therapy, mobilization and oral anticoagulants, up till now mainly VKA, do not always lead to rapid resolution of symptoms or recanalization of venous occlusions, but are associated with long-term disability. Since several years the use of percutaneous transluminal angioplasty (PTA) and stenting in the venous system in patients with outflow obstructions of the iliofemoral veins has gained more attention. PTA and stenting appears to be effective in terms of improvement of PTS symptoms and has shown to be characterized by good mid- to long-term patency rates in mainly observational studies. ${ }^{39-45}$ In acute IFDVT patients in whom catheter directed thrombolysis (CDT) is applied, additional venous stenting immediately following thrombolysis is often deemed indicated in case of underlying venous pathology, such as iliac vein compression syndromes, which may be the cause of the thrombosis in these patients. ${ }^{46}$ Both CDT and stenting procedures are usually followed by anticoagulant therapy with VKA for at least 3 months; patients with PTS usually already use VKA prior to the intervention.

Arterial stenting in combination with antiplatelet therapy has been applied in a larger number of patients for a longer period of time; and as a consequence the body of evidence concerning antithrombotic therapy has grown over time. ${ }^{47}$ However, so far no data evaluating aspects of safety and efficacy for any antithrombotic therapy after venous stenting have been published, resulting in the application of a wide range of anticoagulant (VKA and new oral anticoagulants (NOAC)) and antiplatelet therapies) for different periods of time in day-to-day practice. In chapter 6 of this thesis we present the results of a systematic literature search addressing the issue of antithrombotic therapy after venous stenting. In chapter $\mathbf{7}$ the influence of the quality of anticoagulant treatment with VKA after stent placement was evaluated in terms of stent re- occlusion. Time within therapeutic range (TTR) and the proportion of INR values $<2.0$ were assessed as the main determinants of efficacy in this study. Chapter 8 provides a summary and general discussion of the contents of this thesis. 


\section{References}

1. Versteeg $\mathrm{HH}$, Heemskerk JW, Levi M, Reitsma PH. New fundamentals in hemostasis. Physiological reviews. 2013;93:327-58.

2. Heit JA, Silverstein MD, Mohr DN, Petterson TM, O'Fallon WM, Melton U, 3rd. Risk factors for deep vein thrombosis and pulmonary embolism: a population-based case-control study. Arch Intern Med. 2000;160:809-15.

3. Douketis JD, Berger PB, Dunn AS, Jaffer AK, Spyropoulos AC, Becker RC, et al. The perioperative management of antithrombotic therapy: American College of Chest Physicians Evidence-Based Clinical Practice Guidelines (8th Edition). Chest. 2008;133(6 Suppl):299S-339S.

4. Ansell J, Hirsh J, Hylek E, Jacobson A, Crowther M, Palareti G, et al. Pharmacology and management of the vitamin K antagonists: American College of Chest Physicians Evidence-Based Clinical Practice Guidelines (8th Edition). Chest. 2008;133(6 Suppl):160S-98S.

5. Harder S, Klinkhardt U, Alvarez JM. Avoidance of bleeding during surgery in patients receiving anticoagulant and/or antiplatelet therapy: pharmacokinetic and pharmacodynamic considerations. Clin Pharmacokinet. 2004;43:963-81.

6. Kirkwood TB. Calibration of reference thromboplastins and standardisation of the prothrombin time ratio. Thromb Haemost. 1983;49:238-44.

7. Samama MM, Poller L. Contemporary laboratory monitoring of low molecular weight heparins. Clin Lab Med. 1995;15:119-23.

8. Francis CW, Pellegrini VD, Jr., Totterman S, Boyd AD, Jr., Marder VJ, Liebert KM, et al. Prevention of deep-vein thrombosis after total hip arthroplasty. Comparison of warfarin and dalteparin. The Journal of bone and joint surgery American volume. 1997;79:1365-72.

9. Nieuwenhuis HK, Albada J, Banga JD, Sixma JJ. Identification of risk factors for bleeding during treatment of acute venous thromboembolism with heparin or low molecular weight heparin. Blood. 1991;78:2337-43.

10. Campo G, Pavasini R, Pollina A, Fileti L, Marchesini J, Tebaldi M, et al. Thrombin generation assay: a new tool to predict and optimize clinical outcome in cardiovascular patients? Blood Coagul Fibrinolysis. 2012;23:680-7.

11. Ninivaggi M, Apitz-Castro R, Dargaud Y, de Laat B, Hemker HC, Lindhout T. Whole-blood thrombin generation monitored with a calibrated automated thrombogram-based assay. Clin Chem. 2012;58:1252-9.

12. Linden MWvd, Westert, G.P., Bakker, D. de, Schellevis, F. Tweede Nationale Studie naar ziekten en verrichtingen in de huisartspraktijk: klachten en aandoeningen in de bevolking en in de huisartspraktijk. Utrecht: NIVEL, 2004.

13. Duff J, Walker K, Omari A, Stratton C. Prevention of venous thromboembolism in hospitalized patients: analysis of reduced cost and improved clinical outcomes. Journal of vascular nursing : official publication of the Society for Peripheral Vascular Nursing. 2013;31:9-14.

14. Falck-Ytter Y, Francis CW, Johanson NA, Curley C, Dahl OE, Schulman S, et al. Prevention of VTE in orthopedic surgery patients: Antithrombotic Therapy and Prevention of Thrombosis, 9th ed: American College of Chest Physicians Evidence-Based Clinical Practice Guidelines. Chest. 2012;141(2 Suppl):e278S-325S.

15. Kahn SR, Lim W, Dunn AS, Cushman M, Dentali F, Akl EA, et al. Prevention of VTE in nonsurgical patients: Antithrombotic Therapy and Prevention of Thrombosis, 9th ed: American College of Chest Physicians Evidence-Based Clinical Practice Guidelines. Chest. 2012;141(2 Suppl):e195S-226S.

16. Gould MK, Garcia DA, Wren SM, Karanicolas PJ, Arcelus JI, Heit JA, et al. Prevention of VTE in nonorthopedic surgical patients: Antithrombotic Therapy and Prevention of Thrombosis, 9th ed: American College of Chest Physicians Evidence-Based Clinical Practice Guidelines. Chest. 2012;141(2 Suppl):e227S-77S.

17. Monreal M, Kakkar AK, Caprini JA, Barba R, Uresandi F, Valle R, et al. The outcome after treatment of venous thromboembolism is different in surgical and acutely ill medical patients. Findings from the RIETE registry. J Thromb Haemost. 2004;2:1892-8. 
18. Goldhaber SZ, Tapson VF, Committee DFS. A prospective registry of 5,451 patients with ultrasoundconfirmed deep vein thrombosis. Am J Cardiol. 2004;93:259-62.

19. Tapson VF, Decousus H, Pini M, Chong BH, Froehlich JB, Monreal M, et al. Venous thromboembolism prophylaxis in acutely ill hospitalized medical patients: findings from the International Medical Prevention Registry on Venous Thromboembolism. Chest. 2007;132:936-45.

20. Cohen AT, Tapson VF, Bergmann JF, Goldhaber SZ, Kakkar AK, Deslandes B, et al. Venous thromboembolism risk and prophylaxis in the acute hospital care setting (ENDORSE study): a multinational cross-sectional study. Lancet. 2008;371:387-94.

21. Maynard GA, Morris TA, Jenkins IH, Stone S, Lee J, Renvall M, et al. Optimizing prevention of hospitalacquired venous thromboembolism (VTE): prospective validation of a VTE risk assessment model. J Hosp Med. 2010;5:10-8.

22. Umscheid CA, Hanish A, Chittams J, Weiner MG, Hecht TE. Effectiveness of a novel and scalable clinical decision support intervention to improve venous thromboembolism prophylaxis: a quasiexperimental study. BMC medical informatics and decision making. 2012;12:92.

23. Kucher N, Koo S, Quiroz R, Cooper JM, Paterno MD, Soukonnikov B, et al. Electronic alerts to prevent venous thromboembolism among hospitalized patients. N Engl J Med. 2005;352:969-77.

24. Kucher N, Puck M, Blaser J, Bucklar G, Eschmann E, Luscher TF. Physician compliance with advanced electronic alerts for preventing venous thromboembolism among hospitalized medical patients. J Thromb Haemost. 2009; 7:1291-6.

25. Ahmed I, Gertner E, Nelson WB, House CM, Dahiya R, Anderson CP, et al. Continuing warfarin therapy is superior to interrupting warfarin with or without bridging anticoagulation therapy in patients undergoing pacemaker and defibrillator implantation. Heart Rhythm. 2010;7:745-9.

26. Cano O, Osca J, Sancho-Tello MJ, Olague J, Castro JE, Salvador A. Morbidity associated with three different antiplatelet regimens in patients undergoing implantation of cardiac rhythm management devices. Europace. 2011;13:395-401.

27. Chow V, Ranasinghe I, Lau J, Stowe H, Bannon P, Hendel N, et al. Peri-procedural anticoagulation and the incidence of haematoma formation after permanent pacemaker implantation in the elderly. Heart Lung Circ. 2010;19:706-12.

28. Ghanbari H, Feldman D, Schmidt M, Ottino J, Machado C, Akoum N, et al. Cardiac resynchronization therapy device implantation in patients with therapeutic international normalized ratios. Pacing Clin Electrophysiol. 2010;33:400-6.

29. Krane LS, Laungani R, Satyanarayana R, Kaul S, Bhandari M, Peabody JO, et al. Robotic-assisted radical prostatectomy in patients receiving chronic anticoagulation therapy: role of perioperative bridging. Urology. 2008;72:1351-5.

30. Li HK, Chen FC, Rea RF, Asirvatham SJ, Powell BD, Friedman PA, et al. No increased bleeding events with continuation of oral anticoagulation therapy for patients undergoing cardiac device procedure. Pacing Clin Electrophysiol. 2011;34:868-74.

31. Page SP, Siddiqui MS, Finlay M, Hunter RJ, Abrams DJ, Dhinoja M, et al. Catheter ablation for atrial fibrillation on uninterrupted warfarin: can it be done without echo guidance? J Cardiovasc Electrophysiol. 2011;22:265-70.

32. Tompkins C, Cheng A, Dalal D, Brinker JA, Leng CT, Marine JE, et al. Dual antiplatelet therapy and heparin "bridging" significantly increase the risk of bleeding complications after pacemaker or implantable cardioverter-defibrillator device implantation. J Am Coll Cardiol. 2010;55:2376-82.

33. Eijgenraam $\mathrm{P}$, ten Cate $\mathrm{H}$, ten Cate-Hoek AJ. Practice of bridging anticoagulation: guideline adherence and risk factors for bleeding. Neth J Med. 2014;72:157-64.

34. Douketis JD, Spyropoulos AC, Spencer FA, Mayr M, Jaffer AK, Eckman MH, et al. Perioperative Management of Antithrombotic Therapy: Antithrombotic Therapy and Prevention of Thrombosis, 9th ed: American College of Chest Physicians Evidence-Based Clinical Practice Guidelines. Chest. 2012;141(2 Suppl):e326S-50S.

35. Cannegieter SC, Rosendaal FR, Briet E. Thromboembolic and bleeding complications in patients with mechanical heart valve prostheses. Circulation. 1994;89:635-41.

36. Hart RG, Benavente O, McBride R, Pearce LA. Antithrombotic therapy to prevent stroke in patients with atrial fibrillation: a meta-analysis. Ann Intern Med. 1999;131:492-501. 
37. Douketis JD, Foster GA, Crowther MA, Prins MH, Ginsberg JS. Clinical risk factors and timing of recurrent venous thromboembolism during the initial 3 months of anticoagulant therapy. Arch Intern Med. 2000;160:3431-6.

38. Kahn SR, Shrier I, Julian JA, Ducruet T, Arsenault L, Miron MJ, et al. Determinants and time course of the postthrombotic syndrome after acute deep venous thrombosis. Ann Intern Med. 2008;149:698707.

39. AbuRahma AF, Perkins SE, Wulu JT, Ng HK. Iliofemoral deep vein thrombosis: conventional therapy versus lysis and percutaneous transluminal angioplasty and stenting. Ann Surg. 2001;233:752-60.

40. Titus JM, Moise MA, Bena J, Lyden SP, Clair DG. Iliofemoral stenting for venous occlusive disease. J Vasc Surg. 2011;53:706-12.

41. Sillesen $\mathrm{H}$, Just $\mathrm{S}$, Jorgensen $\mathrm{M}$, Baekgaard $\mathrm{N}$. Catheter directed thrombolysis for treatment of iliofemoral deep venous thrombosis is durable, preserves venous valve function and may prevent chronic venous insufficiency. Eur J Vasc Endovasc Surg. 2005;30:556-62.

42. Kolbel T, Lindh M, Holst J, Uher P, Eriksson KF, Sonesson B, et al. Extensive acute deep vein thrombosis of the iliocaval segment: midterm results of thrombolysis and stent placement. J Vasc Interv Radiol. 2007;18:243-50.

43. Baekgaard N, Broholm R, Just S, Jorgensen M, Jensen LP. Long-term results using catheter-directed thrombolysis in 103 lower limbs with acute iliofemoral venous thrombosis. Eur J Vasc Endovasc Surg. 2010;39:112-7.

44. Manninen H, Juutilainen A, Kaukanen E, Lehto S. Catheter-directed thrombolysis of proximal lower extremity deep vein thrombosis: a prospective trial with venographic and clinical follow-up. Eur J Radiol. 2012;81:1197-202.

45. Sharifi M, Mehdipour M, Bay C, Smith G, Sharifi J. Endovenous therapy for deep venous thrombosis: the TORPEDO trial. Catheter Cardiovasc Interv. 2010;76:316-25.

46. Kim JY, Choi D, Guk Ko Y, Park S, Jang Y, Lee do Y. Percutaneous treatment of deep vein thrombosis in May-Thurner syndrome. Cardiovasc Intervent Radiol. 2006;29:571-5.

47. Vik-Mo H, Slette M, Hegbom K. [Antithrombotic therapy after percutanous coronary intervention with stenting]. Tidsskrift for den Norske laegeforening : tidsskrift for praktisk medicin, ny raekke. 2008;128:436-9. 


\section{Chapter 2}

The effect of clinical decision support on adherence to thrombosis prophylaxis guidelines in medical patients; a single center experience

Pieter Eijgenraam, Nathalie Meertens, René van den Ham, Hugo ten Cate, Arina J ten Cate-Hoek Thromb Res. 2015;135: 464-471 


\begin{abstract}
Introduction

Venous thromboembolism (VTE) is an underestimated health problem. The administration of low molecular weight heparins (LMWH) to the appropriate patients dramatically decreases VTE incidence. Clinical decision support (CDS) might contribute to thrombosis prophylaxis guideline adherence.
\end{abstract}

\title{
Methods
}

A computerized integrated risk score program was used to estimate VTE and bleeding risk of nonsurgical patients. A VTE risk score of $\geq 4$ resulted in an advice to administer LMWH. We selected 64 medical patients before the introduction of CDS (TO) and 64 patients after the introduction (T1). We compared guideline compliance between these groups using chi $^{2}$ tests.

\section{Results}

No difference between groups was found; Adherence to the guidelines at T0 was $59.4 \%$, the same percentage of $59.4 \%$ was found at T1. To evaluate the effect of the introduction of CDS in terms of under and overtreatment we compared the prevalence of over and under treatment at $\mathrm{T} 1$ and T0. The OR for receiving under treatment at T1 compared to T0 is $0.48(95 \% \mathrm{Cl}$ : $0.18-1.30), p=0.14$. The OR for overtreatment at T1 compared to TO is 1.66 (95\% Cl: 0.74-3.73), $\mathrm{p}=0.22$

\section{Conclusion}

We found no improvement in guideline adherence towards anti thrombotic prophylaxis in medical patients after the introduction of CDS in this pilot study. There was however a nonsignificant shift towards over treatment. Possible explanations for these results are the increased awareness of the risk for thromboembolism induced by the study, suboptimal use of CDS and deviation from CDS advice caused by patient's preferences. 


\section{Introduction}

Deep venous thrombosis (DVT) and pulmonary embolism (PE) collectively referred to as venous thromboembolism (VTE) represent a major health problem for hospitalized patients. The yearly incidence of DVT in The Netherlands is 0.6-1.2 cases per 1000 inhabitants. ${ }^{1}$ The reported VTE incidence in hospitalized patients is 100 times greater. ${ }^{2}$ Currently, antithrombotic prophylaxis with low molecular weight heparins (LMWH) or in some cases unfractionated heparin is applied to prevent VTE. The positive effect of antithrombotic prophylaxis in general surgical, orthopedic surgical and nonsurgical patients on the incidence of VTE has been firmly established in different studies. ${ }^{3-5}$ However, literature provides evidence supporting the thesis that antithrombotic prophylaxis is underused in the hospital setting, leading to avoidable cases of VTE. Different studies demonstrated that in only $30-50 \%$ of the patients indicated for prophylaxis appropriate measures are indeed taken. ${ }^{6-8}$ The administration of appropriate prophylaxis in medical patients is observed to be even less than in surgical patients. ${ }^{9}$ Initiatives promoting the use of clinical decision support systems (CDS) or simple electronic alerts have gained more and more attention and have proven efficacy in terms of adherence to guidelines and in some cases reduction of VTE incidence. ${ }^{10-14}$ Especially in institutions such as university hospitals, with a high throughput of inexperienced medical personnel in combination with a complex patient load, CDS could function as a guide for the management of antithrombotic prophylaxis.

We assessed whether the introduction of a computer based CDS embedded in the hospital patient data system might lead to improved adherence to guidelines for antithrombotic prophylaxis in medical patients.

\section{Methods}

\section{CDS}

From September $1^{\text {st }}$ to December $1^{\text {st }} 2013$ a pilot study was performed in the Maastricht University Medical Centre (MUMC+) on the introduction, use and evaluation of a computer based CDS. Institutional review board approval was obtained (METC 13-5-034). Before the introduction of CDS the application of thromboprophylaxis was left to the discretion of the physician, who could consult locally available web based protocols (ODIN), based on international guidelines. By using CDS a protocol based VTE prophylaxis advice is generated, avoiding the need of 
consulting the underlying protocol by the prescribing physician. A customized computerized integrated risk score program was used to estimate VTE and bleeding risk of nonsurgical patients, as described below. All physicians involved in the pilot study were collectively informed about the use and function of CDS and were motivated to participate. The first risk assessment for all admitted medical patients was performed within 24 hours after hospitalization and was hereafter repeated daily. The CDS was installed on four stand-alone personal computers on two wards of the Maastricht University Medical Centre (MUMC+). The following inclusion criteria were applied: 1) the patient is non-surgical and admitted to one of the two participating wards and 2) the (expected time) of admission is at least 48 hours. Excluded were 1) patients on therapeutic anticoagulants and 2) patients with active bleeding.

The hospital patient data system (SAP, Germany) menu was extended with a dedicated CDS button on the opening page of the patient's record. The use of this button was not mandatory and if CDS generated a recommendation for antithrombotic prophylaxis, no automatic link to the pharmacotherapeutic ordering system was provided.

The Padua Prediction Score ${ }^{11}$ for VTE risk factors, endorsed by the ACCP guidelines $2012^{4}$, was used to compose the CDS data form. This risk assessment model (RAM), prospectively validated in a study with patients not receiving prophylaxis ${ }^{15}$ awards each risk factor with a maximum of 3 points. Scores of the different risk factors are computed into a total VTE risk score, by simple addition. VTE risk was considered high at a score of 4 points or more. The bleeding risk was assessed using a non-validated RAM. ${ }^{16}$ With a bleeding score of 7 or more points the patient was considered at high risk of bleeding. Both the risk for thrombosis and the risk for bleeding were dichotomized. A VTE risk score of $<4$ resulted in an advice not to administer prophylaxis, a VTE score of $\geq 4$ led to an advice to administer (weight adjusted) LMWH. A bleeding score of $>7$ resulted in a warning in CDS that bleeding risk was high, in case the score $\leq 7$ points the announcement 'low bleeding risk' was depicted within CDS.

\section{Assessment of compliance}

In the period prior to the introduction of the CDS, when only the MUMC+ protocol, mainly based on ACCP 2008 guidelines, was available for physicians as a guide to prescribe the correct antithrombotic prophylaxis, compliance to antithrombotic guidelines was assessed on two randomly selected dates for baseline measurements (T0). The measurements were repeated on two randomly selected dates towards the end of the CDS pilot period (T1). For an overview of the patient flow and measurements see Figure 2.1. All risk factors for VTE and bleeding included in the 
MUMC+ protocol (T0) and the CDS RAMs (T1) were recorded by two independent researchers for all individual patients admitted to one of the participating wards on the day that the sample was taken. The appropriate antithrombotic prophylaxis was established based on the risk stratification methods at hand. In the MUMC+ protocol VTE risk factors are indicated, but no individual weights are awarded and no risk factors for bleeding are stated. In case of perceived increased bleeding risk the advice to administer reduced doses of $\mathrm{LMWH}$, or no $\mathrm{LMWH}$, is given. For the patients assessed at T1 compliance was assessed using the CDS RAMs; for an overview of the risk factors, see Appendix 2.1. For the assessment at TO all medical records were reviewed, for risk assessment at $\mathrm{T} 1$, medical records were reviewed in case the prescribing physician did not use CDS on the sampling day. Pharmacy records were assessed to verify whether the CDS-generated advice on prophylaxis, resulted in the actual administration of the appropriate prophylaxis. Bed rest, generally poorly reported in medical records, was recorded after interviews with nurses of the participating wards on each date of measurement. Guideline adherence was defined as follows: the patient receives the appropriate prophylaxis (pharmacological or no prophylaxis) on the day the sample is taken.

Figure 2.1 Patient flow and measurements

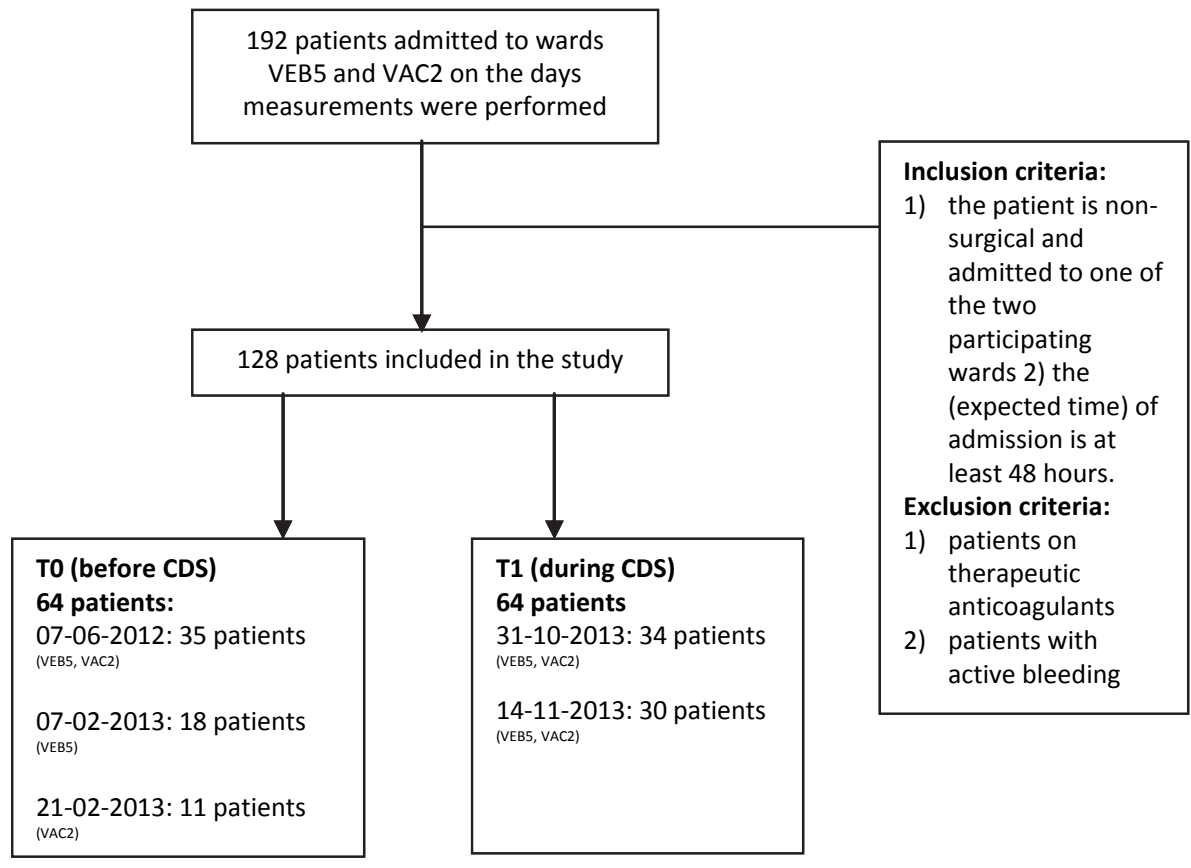


In case guidelines were not followed, the following 2 options were recorded; 1 ) Under treatment defined as not receiving $\mathrm{LMWH}$, while an indication was present; 2) over treatment defined as receiving LMWH without indication.

\section{Evaluation of CDS use}

After termination of the pilot we evaluated the use of CDS; a questionnaire consisting of 4 domains was designed to identify possible barriers for CDS use. The questionnaire is based on perceived barriers impeding guideline adherence among Dutch general practitioners. ${ }^{17,18}$ We identified barriers related to knowledge (e.g. lack of awareness of CDS, lack of familiarity with CDS), barriers related to attitude (e.g. lack of outcome expectancy, lack of motivation), barriers related to behaviour (e.g. patients' preferences not matching recommendations), and environmental factors (e.g. lack of education, lack of time). See Appendix 2.2 for the complete questionnaire. We asked the physicians who worked with CDS to complete the questionnaire anonymously.

\section{Statistical analysis}

To detect an improvement in guideline adherence from $50 \%$ to $75 \%$, with a power of $80 \%, 128$ (T0: 64, T1: 64) participants were needed. Cumulative first use of CDS of the participating wards is reported. Descriptive statistics were used to determine patient characteristics. Continuous variables are reported as means and their standard deviations (SD); categorical data are presented as counts and percentages. Chi ${ }^{2}$ tests are used to compare categorical variables including the compliance to the antithrombotic prophylaxis guidelines before and after the introduction of CDS. To estimate the strength of the association between the introduction of CDS and observed compliance Phi statistics are used. Student's t-tests were used to compare continuous variables. Under and over treatment was separately assessed. The associations are also expressed as odds ratios (OR) with accompanying confidence intervals $(\mathrm{Cl})$. A two sided $\mathrm{p}$-value $<0.05$ is considered statistically significant. Data were analyzed with SPSS version 22.

\section{Results}

The cumulative rate of first use of CDS in days was explored for the full 3 months duration of the pilot. The software that analyzed the cumulative rate of first use was not designed to differentiate between medical and surgical patients; therefore this rate is only an indication for CDS use in medical patients. Results for the 2 different 
wards (VEB5 and VAC2) are depicted in Figure 2.2. Ward VEB5 consists of predominantly medical patients; VAC2 is a ward populated by both medical and surgical patients, only medical patients were assessed.

Figure 2.2 Cumulative rate of first CDS use in time (days); ward VEB5 and VAC2

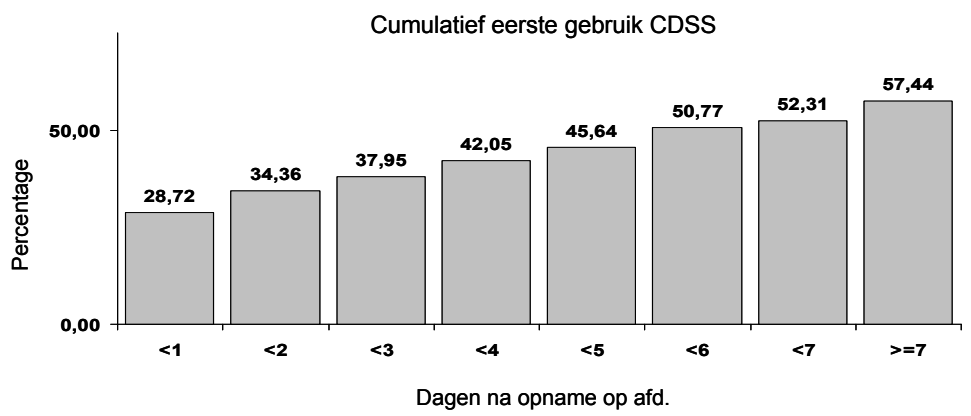

We included 128 patients; 64 patients were included in the baseline measurement (T0), and 64 patients were included in the post CDS introduction measurement (T1). For detailed patient characteristics of included patients at T0 and T1 see Table 2.1. Anticipated bed rest with bathroom privileges for at least 3 days, a major risk factor, was prescribed to $17.2 \%$ at $\mathrm{T} 0$ and $3.1 \%$ at $\mathrm{T} 1$. In our two random samples the medical records of in total 96 patients were reviewed (all 64 patients on T0, and 64 minus 32 CDS assessed patients at T1). The CDS score lists were completed on the day the sample was taken in $50.0 \%$ of the cases; the remaining patients were evaluated without CDS (17.2\%), or on the basis of a CDS advice which was calculated on previous days (32.8\%).

According to the local protocol at T0, $45.3 \%$ of the patients should have received pharmacological prophylaxis, $6.3 \%$ mechanical prophylaxis and $48.4 \%$ of patients were not eligible for any thromboprophylaxis. Pharmacological prophylaxis was administered in $50.0 \%$ of the cases; mechanical prophylaxis was rarely used, in only $1.6 \%$ of the patients.

According to the RAMs for VTE and bleeding risk assessment at T1 $21.9 \%$ of the patients should have received pharmacological prophylaxis, $6.3 \%$ mechanical prophylaxis and $\mathbf{7 1 . 9 \%}$ were not eligible for any antithrombotic prophylaxis. The percentage of patients that received pharmacologic prophylaxis was more than twice as high (45.3\%), and mechanical prophylaxis was not used. For an overview of the prescribed antithrombotic treatment modalities see Table 2.2. Adherence to the 
guidelines at T0 was $59.4 \%$, the same percentage of $59.4 \%$ was found at $\mathrm{T} 1$, resulting in a Pearson's $\chi^{2}$ of $0.00 ; p$-value $=1.00$.

Table 2.1 Patient characteristics

\begin{tabular}{|c|c|c|c|}
\hline & T0: 64 patients & T1: 32 of 64 patients $^{a}$ & p-value \\
\hline Age & 66.3 (SD: 19.3) & 69.2 (SD: 15.1) & 0.45 \\
\hline Sex & $27(45.3 \%)$ male & 19 (59.4 \%) male & 0.11 \\
\hline Weight & 77.3 kg (SD: 17.6) & 67.9 kg (SD: 20.2) & 0.02 \\
\hline $\mathrm{BMI}>30$ & $11(17.2 \%)$ & $5(15.6 \%)$ & 0.85 \\
\hline History VTE & $2(3.1 \%)$ & $5(15.6 \%)$ & 0.03 \\
\hline Known thrombophilia & $0(0 \%)$ & $0(0 \%)$ & 0.73 \\
\hline Congestive heart disease and/or COPD & $27(42.2 \%)$ & $9(28.1 \%)$ & 0.18 \\
\hline Acute infectious and/or rheumatic disorder & $35(54.7 \%)$ & $13(40.6 \%)$ & 0.19 \\
\hline Active gastro duodenal ulcer & $6(9.4 \%)$ & $2(6.3 \%)$ & 0.60 \\
\hline Current cancer & $9(14.1 \%)$ & $6(18.8 \%)$ & 0.55 \\
\hline Hepatic failure and INR >1.5 & $6(9.4 \%)$ & $0(0 \%)$ & 0.15 \\
\hline Platelet count $<50 \times 10^{9}$ cells $/$ I & $2(3.1 \%)$ & $0(0 \%)$ & 0.64 \\
\hline eGFR (creatinine clearance) $<60 \mathrm{ml} / \mathrm{m}(<30 \mathrm{ml} / \mathrm{l})$ & $23(2)(35.9 \%(3.1 \%))$ & $10(3)(31.3 \%(9.4 \%))$ & $0.65(0.19)$ \\
\hline Bed rest $\geq 2$ days & $11(17.2 \%)$ & $1(3.1 \%)$ & 0.05 \\
\hline Bleeding < 3 months before admission & $10(16.7 \%)$ & $10(31.3 \%)$ & 0.08 \\
\hline Trauma/surgery legs, hips, pelvis $<1$ month & $3(4.7 \%)$ & $1(3.1 \%)$ & 0.72 \\
\hline
\end{tabular}

${ }^{a}$ At T1 medical records were only reviewed in case the prescribing physician did not use CDS on the sampling day. Abbreviations: BMI, body mass index; COPD, chronic obstructive pulmonary disease; eGFR, estimated glomerular filtration rate; INR, international normalized ratio; SD, standard deviation, VTE, venous thromboembolism

Table 2.2 Prescribed antithrombotic treatment

\begin{tabular}{lll}
\hline & T0: 64 patients & T1: 64 patients \\
\hline Pharmacological prophylaxis & & \\
Nadroparin 2,850 IU & $21(32.8 \%)$ & $20(31.3 \%)$ \\
Nadroparin 3,800 IU & $1(1.6 \%)$ & $3(4.7 \%)$ \\
Nadroparin 5,700IU & $5(7.8 \%)$ & $3(4.7 \%)$ \\
Nadroparin 7,600IU & $0(0 \%)$ & $1(1.6 \%)$ \\
Tinzaparin 10,000 IU & $2(3.1 \%)$ & $0(0.0 \%)$ \\
Tinzaparin 14,000 IU & $3(4.7 \%)$ & $1(1.6 \%)$ \\
Tinzaparin 18,000 IU & $0(0 \%)$ & $1(1.6 \%)$ \\
Mechanical prophylaxis & & $0(0.0 \%)^{\mathrm{a}}$ \\
graduated compression stockings (GCS) & $1(1.6 \%)($ received also & \\
intermittent pneumatic compression therapy (IPC) & $0(0.0 \%)$ & $0(0.0 \%)^{\mathrm{a}}$ \\
\hline
\end{tabular}

${ }^{a}$ At T1 medical records were only reviewed in case the prescribing physician did not use CDS on the sampling day (32 patients); LMWH use was assessed in all cases. Abbreviations: IE, international units

For the 64 patients at T0 protocol was not followed in 26 cases; 13 patients $(13 / 64=20.3 \%)$ did not receive antithrombotic prophylaxis while they did have an indication for prophylaxis. The other 13 patients $(13 / 64=20.3 \%)$ of this group were treated with antithrombotic prophylaxis without indication. In the 64 patients at T1 
also deviation from protocol was found in 26 patients; in this group 7 patients (7/64=10.9\%) were undertreated and 19 patients (19/64=29.7\%) were over treated; see Table 2.3. To evaluate the effect of the introduction of CDS in terms of under and overtreatment we compared the prevalence of over and under treatment at $\mathrm{T} 1$ and T0. The OR for under treatment at T1 compared to T0 is 0.48 (95\% Cl: $0.18-1.30)$, $\mathrm{p}=0.14$. The OR for receiving overtreatment at T1 compared to T0 is $1.66(95 \% \mathrm{Cl}$ : 0.74-3.73), $p=0.22$. A summary of the comparison between T0 and T1 in terms of adherence can be found in Table 3. When the clinicians made use of CDS assistance in $12.5 \%(4 / 32)$ of the cases patients did not receive the actual LMWH dose prescribed by CDS.

Table 2.3 Comparison of guideline compliance between T0 and T1

\begin{tabular}{|c|c|c|c|c|}
\hline & T0: 64 patients & & T 1: 64 patients & \\
\hline $\begin{array}{l}\% \text { of the group in } \\
\text { need of } \\
\text { antithrombotic } \\
\text { measure according } \\
\text { to MUMC protocol }\end{array}$ & $\begin{array}{l}\text { Pharmacologic: } \\
45.3 \%(29 / 64) \\
\text { No prophylaxis: } \\
54.7 \%(35 / 64)\end{array}$ & $\begin{array}{l}\% \text { of the group in } \\
\text { need of } \\
\text { antithrombotic } \\
\text { measure according } \\
\text { to RAMs }\end{array}$ & $\begin{array}{l}\text { Pharmacologic: } \\
21.9 \%(14 / 64) \\
\text { No prophylaxis: } \\
78.1 \%(50 / 64)\end{array}$ & \\
\hline $\begin{array}{l}\text { \% of the group } \\
\text { actually received } \\
\text { prophylaxis }\end{array}$ & $\begin{array}{l}\text { Pharmacologic: } \\
50 \%(32 / 64) \\
\text { No prophylaxis: } \\
50 \%(32 / 64)\end{array}$ & $\begin{array}{l}\% \text { of the group } \\
\text { actually received } \\
\text { prophylaxis }\end{array}$ & $\begin{array}{l}\text { Pharmacologic: } \\
45.3 \%(29 / 64) \\
\text { No prophylaxis: } \\
54.7 \%(35 / 64)\end{array}$ & \\
\hline $\begin{array}{l}\% \text { of the group } \\
\text { Compliant to } \\
\text { protocol }\end{array}$ & $59.4 \%(38 / 64)$ & $\begin{array}{l}\% \text { of the group } \\
\text { compliant to RAMs }\end{array}$ & $59.4 \%(38 / 64)$ & $P=1.00$ \\
\hline $\begin{array}{l}\% \text { of the group } \\
\text { under treated }\end{array}$ & $20.3 \%(13 / 64)$ & $\begin{array}{l}\% \text { of the group } \\
\text { under treated }\end{array}$ & $10.9 \%(7 / 64)$ & $\begin{array}{l}\text { OR for under } \\
\text { treatment at T 1: } \\
0.48 \text { ( } 95 \% \mathrm{Cl}: 0.18- \\
1.30)\end{array}$ \\
\hline $\begin{array}{l}\% \text { of the group over } \\
\text { treated }\end{array}$ & $20.3 \%(13 / 64)$ & $\begin{array}{l}\% \text { of the group over } \\
\text { treated }\end{array}$ & $29,7 \%(19 / 64)$ & $\begin{array}{l}\text { OR for over } \\
\text { treatment at T 1: } \\
1.66(95 \% \mathrm{Cl}: 0.74- \\
3.73)\end{array}$ \\
\hline
\end{tabular}

Abbreviations: MUMC, Maastricht University Medical Centre+; RAM, Risk Assessment Model

Finally, we analyzed the outcomes of the questionnaire presented to all CDS users. Five physicians completed this online questionnaire. All participating physicians estimated their knowledge of CDS as sufficient; they were all aware of the fact that CDS was introduced, all had enough software knowledge of CDS use, all received proper instructions and 4/5 physicians rated their knowledge of the patient's risk factors for thrombosis and bleeding as generally sufficient. Barriers related to attitude were perceived: 2/5 participants questioned whether CDS generated the correct VTE 
prophylaxis advice for complex patients with several co-morbidities, the average score for experiencing difficulties due to the introduction of CDS (1: 'no problem'-5: 'very difficult') was scored 2.6 (SD: 1.5), 3/5 did not know whether CDS was 'evidence based', 4/5 perceived CDS advices as clear and 4/5 thought that the use of CDS would lead to better patients outcomes. We also inquired after behavioural aspects towards CDS; the question whether patient's preferences influenced the decision to deviate from the CDS advice, was answered with an average 2.4 (SD: 0.5) on a scale from 1 'never' to 5 'very often' and of all participants 2 preferred to use CDS for high-complex patients and 3 preferred to use CDS in both high and low-complex patients. Finally we inquired after environmental factors regarding the CDS use: all participants judged that the use of CDS required a substantial additional time investment and 2/5 had the opinion that a direct link to the ordering system would lead to a decrease in mistakes with regard to $\mathrm{LMWH}$ administration.

\section{Discussion}

We found no improvement in guideline adherence towards anti thrombotic prophylaxis in medical patients after the introduction of CDS in this pilot study. Guidelines were followed in $59.4 \%$ both before and after the introduction of CDS. There was however a non-significant shift towards over treatment, which may be indicative of higher prophylaxis awareness. The finding that CDS did not result in higher guideline adherence is not coherent with results presented in other studies. Several studies demonstrated a positive, often temporary effect on adherence caused merely by the fact that the introduction of CDS was accompanied by increased awareness of the importance of VTE prevention. The introduction of CDS is associated with increased rates of per protocol administration of VTE prophylaxis, increased rates of administration of VTE prophylaxis in general ${ }^{14}$ and even with reduced rates of VTE. ${ }^{13}$ The observed lack of improvement in adherence in this pilot study could, at least partially, be caused by the suboptimal use of CDS. Final use of CDS varied between $23.7 \%$ and $57.4 \%$ for the different wards. A barrier towards implementation of CDS could have been the additional time investment needed as indicated by physicians in the questionnaire; moreover, time consuming separate login procedures were required in order to enter CDS. Doubts whether CDS was based on solid evidence, uncertainty about the correctness of CDS advices for 'complex' patients, experienced difficulties due to the introduction of CDS and deviation from CDS advice caused by patient's preferences as indicated in the questionnaire also might have attributed to the perceived lack of improvement in adherence. 
Our study has several weaknesses. In the first place the sample size was small; only 128 patients participated in this pilot study and no follow-up VTE incidences were assessed. Therefore no association with the incidence of VTE could be made. Secondly, VTE risks for patients at TO were determined using a local protocol that did not include a risk score for bleeding. The RAM used at T1 included a calculated score for bleeding risk. This could have led to a difference in risk perception. The fact that use of CDS required time consuming login procedures and the fact that no link was provided to the ordering system may have been the most important barrier, resulting in the limited impact of CDS in this study.

In contrast to findings in other studies we conclude that introduction of CDS did not have a positive impact on guideline adherence. A non-significant shift towards over treatment was observed following the introduction of CDS. An easily accessible and mandatory CDS linked to the electronic pharmacy system might be needed in order to improve guideline adherence and associated reduction in VTE incidence. 


\section{References}

1. Linden MWvd, Westert GP, Bakker D de, Schellevis F. Tweede Nationale Studie naar ziekten en verrichtingen in de huisartspraktijk: klachten en aandoeningen in de bevolking en in de huisartspraktijk. Utrecht: NIVEL, 2004.

2. Duff J, Walker K, Omari A, Stratton C. Prevention of venous thromboembolism in hospitalized patients: analysis of reduced cost and improved clinical outcomes. J Vasc Nurs. 2013;31:9-14.

3. Falck-Ytter Y, Francis CW, Johanson NA, Curley C, Dahl OE, Schulman S, et al. Prevention of VTE in orthopedic surgery patients: Antithrombotic Therapy and Prevention of Thrombosis, 9th ed: American College of Chest Physicians Evidence-Based Clinical Practice Guidelines. Chest. 2012;141(2 Suppl):278-325.

4. Kahn SR, Lim W, Dunn AS, Cushman M, Dentali F, Akl EA, et al. Prevention of VTE in nonsurgical patients: Antithrombotic Therapy and Prevention of Thrombosis, 9th ed: American College of Chest Physicians Evidence-Based Clinical Practice Guidelines. Chest. 2012;141:195-226.

5. Gould MK, Garcia DA, Wren SM, Karanicolas PJ, Arcelus JI, Heit JA, et al. Prevention of VTE in nonorthopedic surgical patients: Antithrombotic Therapy and Prevention of Thrombosis, 9th ed: American College of Chest Physicians Evidence-Based Clinical Practice Guidelines. Chest. 2012;141:227-77.

6. Monreal M, Kakkar AK, Caprini JA, Barba R, Uresandi F, Valle R, et al. The outcome after treatment of venous thromboembolism is different in surgical and acutely ill medical patients. Findings from the RIETE registry. J Thromb Haemost. 2004;2:1892-8.

7. Goldhaber SZ, Tapson VF, Committee DFS. A prospective registry of 5,451 patients with ultrasoundconfirmed deep vein thrombosis. Am J Cardiol. 2004;93:259-62.

8. Tapson VF, Decousus H, Pini M, Chong BH, Froehlich JB, Monreal M, et al. Venous thromboembolism prophylaxis in acutely ill hospitalized medical patients: findings from the International Medical Prevention Registry on Venous Thromboembolism. Chest. 2007;132:936-45.

9. Lecumberri R, Marques M, Panizo E, Alfonso A, Garcia-Mouriz A, Gil-Bazo I, et al. High incidence of venous thromboembolism despite electronic alerts for thromboprophylaxis in hospitalised cancer patients. Thromb Haemost. 2013;110:184-90.

10. Kucher N, Puck M, Blaser J, Bucklar G, Eschmann E, Luscher TF. Physician compliance with advanced electronic alerts for preventing venous thromboembolism among hospitalized medical patients. J Thromb Haemost. 2009; 7:1291-6.

11. Kucher N, Koo S, Quiroz R, Cooper JM, Paterno MD, Soukonnikov B, et al. Electronic alerts to prevent venous thromboembolism among hospitalized patients. N Engl J Med. 2005;352:969-77.

12. Baroletti S, Munz K, Sonis J, Fanikos J, Fiumara K, Paterno M, et al. Electronic alerts for hospitalized high-VTE risk patients not receiving prophylaxis: a cohort study. J Thromb Thrombolysis. 2008;25: 146-50.

13. Maynard GA, Morris TA, Jenkins IH, Stone S, Lee J, Renvall M, et al. Optimizing prevention of hospitalacquired venous thromboembolism (VTE): prospective validation of a VTE risk assessment model. J Hosp Med. 2010;5:10-8.

14. Umscheid CA, Hanish A, Chittams J, Weiner MG, Hecht TE. Effectiveness of a novel and scalable clinical decision support intervention to improve venous thromboembolism prophylaxis: a quasiexperimental study. BMC Med Inform Decis Mak. 2012;12:92.

15. Barbar S, Noventa F, Rossetto V, Ferrari A, Brandolin B, Perlati M, et al. A risk assessment model for the identification of hospitalized medical patients at risk for venous thromboembolism: the Padua Prediction Score. J Thromb Haemost. 2010;8:2450-7.

16. Decousus H, Tapson VF, Bergmann JF, Chong BH, Froehlich JB, Kakkar AK, et al. Factors at admission associated with bleeding risk in medical patients: findings from the IMPROVE investigators. Chest. 2011;139:69-79.

17. Lugtenberg M, Burgers JS, Besters CF, Han D, Westert GP. Perceived barriers to guideline adherence: a survey among general practitioners. BMC Fam Pract. 2011;12:98. 
The effect of clinical decision support on adherence to thrombosis prophylaxis guidelines

18. Lugtenberg M, Zegers-van Schaick JM, Westert GP, Burgers JS. Why don't physicians adhere to guideline recommendations in practice? An analysis of barriers among Dutch general practitioners. Implement Sci. 2009;4:54. 


\section{Appendix 2.1 Example of a completed CDS form}

\section{Risk factors VTE}

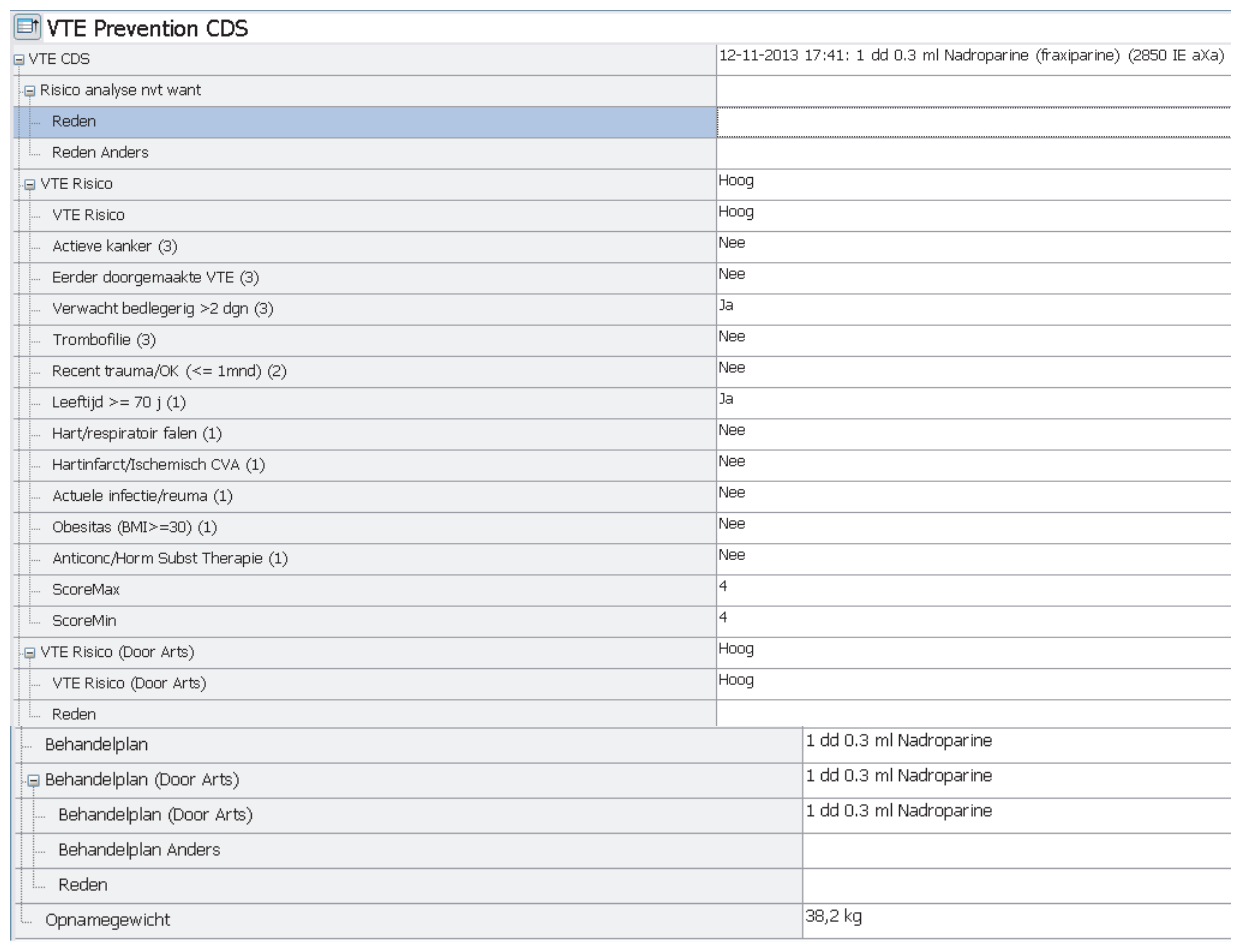

\section{Risk factors bleeding}

\begin{tabular}{|c|c|}
\hline Bloedingsrisico & \\
\hline Bloedingsrisico & Laag \\
\hline Nierfalen $(1-2,5)$ & Nee \\
\hline Leeftijd Categorie $(1,5-3,5)$ & Leeftijd $>=85$ \\
\hline Actieve kanker (2) & Nee \\
\hline Centrale veneuze catheter (2) & Nee \\
\hline Intensive care $(2,5)$ & Nee \\
\hline Leverfalen (INR > 1,5) (1) & Nee \\
\hline Bloedplaatjes < $50 \times 10 \sim 9 /$ (4) & Nee \\
\hline ScoreMin & 3,5 \\
\hline Bloedingsrisico (Door Arts) & Laag \\
\hline Denk aan gebruik plaatjesremmers & \\
\hline Bloedingsrisico (Door Arts) & Laag \\
\hline
\end{tabular}




\section{Appendix 2.2 Questionnaire for evaluation of CDS (in Dutch)}

\section{Kennis}

1) Was u in de periode dat de pilot CDS VTE prevention actief was (1-9-13/1-12-13) op de hoogte van het bestaan van CDS op uw afdeling?
a. Ja

b. Nee

2) Zo ja, had u voldoende kennis van de software om het programma op de juiste wijze te kunnen gebruiken?
a. Ja
b. Nee

3) Heeft u voldoende instructie over het gebruik van CDS ontvangen?

a. Ja

b. Nee

4) Had u bij gebruik van CDS doorgaans voldoende kennis van de patient om alle risicofactoren (trombose/bloeding) op de juiste wijze in het systeem in te voeren?
a. Ja
b. Nee

Attitude

5) Leidt het gebruik van CDS tot een juist behandeladvies voor complexe patienten met veel comorbiditeit, zoals die veelvuldig op uw afdeling voorkomen?
a. Ja

b. Nee

6) Maakte u voor de introductie van de pilot gebruik van het ODIN protocol om op de juiste wijze tromboprofylaxe voor te schrijven?
a. Ja
b. Nee

7) Was het moeilijk over te stappen naar een nieuwe vorm (CDS) van inschatting van het tromboserisico?

Geef een score van 1-5, waarbij 1= geen enkel probleem en 5=zeer lastig.
1

\begin{abstract}
2
\end{abstract}
3
4
5 (een score omcirkelen)

8) Was CDDS in de gehanteerde vorm (pilot) voldoende 'evidence based'?
a. Ja
b. Nee
c. Weet ik niet

9) De door CDS gegenereerde aanbevelingen: (meerdere alternatieven mogen aangekruist)
a. Zijn duidelijk
b. Wegen alle relevante factoren mee in het behandeladvies
c. Zijn 'up to date'
d. Zijn makkelijk toepasbaar

10) Denkt u dat het gebruik van CDS leidt tot betere patientenuitkomsten m.b.t. DVT-preventie vergeleken met de 'oude' situatie?
a. Ja
b. Nee

\section{Gedrag}

11) Speelt de persoonlijke voorkeur van de patient een rol bij het afwijken van volgens protocol voor te schrijven LMWH?

Geef een score van 1-5, waarbij 1= nooit en $5=$ zeer vaak.
1
2
3
4
5 (een score omcirkelen) 
12) Hoe vaak heeft u ten tijde van de pilot gebruik gemaakt van CDS bij het bepalen of een patient tromboprofylaxe nodig heeft of niet?
a. Nooit
b. In ongeveer $0-25 \%$ van de gevallen
c. In ongeveer $25-50 \%$ van de gevallen
d. In ongeveer $50-75 \%$ van de gevallen
e. In ongeveer $75-100 \%$ van de gevallen
f. Altijd

13) Ik heb het meest gebruik gemaakt van CDS voor:
a. Mobiele, laagcomplexe patienten met een op het oog laag DVT risico
b. Meer hoogcomplexe patienten
c. Zowel de hoog- als laagcomplexe patienten

\section{Omgevingsfactoren}

14) Hoe beoordeelt $u$ het gebruiksgemak van CDS:

Geef een score van 1-5, waarbij 1=zeer gebruiksvriendelijk, 5=zeer gebruiksonvriendelijk
1
2
3
4
5 (een score omcirkelen)

15) Open vraag: Op welke wijze zou de gebruiksvriendelijkheid van CDS kunnen worden verbeterd? Antwoord:

16) Denkt u dat een directe koppeling tussen CDS en het medicijnbestelsysteem EVS zou leiden tot minder onjuiste voorschrijvingen/onjuiste doseringen van LMWHs.
a. Ja
b. Nee

17) Vergt het gebruik van CDS een belangrijke extra tijdsinvestering?

a. Ja

b. Nee

18) Heeft CDS in deze vorm een toegevoegde waarde? Geef een score van 1-5, waarbij 1=zeer zeker, 5=zeer zeker niet $\begin{array}{lllll}1 & 2 & 3 & 4 & 5 \text { (een score omcirkelen) }\end{array}$ 


\section{Chapter 3}

Safety and efficacy of bridging with low molecular weight heparins: a systematic review and partial meta-analysis 


\begin{abstract}
Background

Surgical interventions in patients on long term vitamin $\mathrm{K}$ antagonist (VKA) treatment create a dilemma; periprocedural interruption of anticoagulation raises the risk of thrombosis, while continuation raises the risk of bleeding. The anticoagulation-free interval is minimized by "bridging" with parenteral anticoagulants. The efficacy and safety of bridging with low molecular weight heparins (LMWH) has however not been unequivocally established.
\end{abstract}

\title{
Methods
}

We performed an EMBASE and MEDLINE search for studies that compared bridging anticoagulation with continuation or cessation of VKA without bridging; with thromboembolism (TE) and bleeding as outcomes. We identified 878 articles and finally selected 17 . Results of individual studies were pooled.

\section{Results}

None of the included studies reported significant differences in incidence of TEs between the bridging group and the comparator group; 4 out of 13 studies reported zero TEs. Heparin was identified as a risk factor for bleeding in multivariable adjusted analyses in 3 studies on pacemaker/implantable cardioverter defibrillator (PM/ICD) surgery. In 5 studies (different types of surgery) with unadjusted analyses, bridging was compared to warfarin cessation: 3 studies reported null results for bleeding; 2 studies identified bridging as a risk factor. We pooled a subset of 6 studies regarding postoperative bleeding after PM/ICD surgery and found a relative risk (RR) of 3.03 (95\% confidence interval (Cl), 1.86-4.95) for bridging compared to continuation of VKA.

\section{Conclusions}

While the antithrombotic efficacy of bridging with LMWH has not been demonstrated, increased bleeding risk is observed in different types of surgery. PM/ICD surgery can be safely performed on continued VKA. 


\section{Background}

Vitamin $\mathrm{K}$ antagonists (VKA) still are commonly used agents in the prevention of venous or arterial thromboembolism (TE). Annually, approximately $10 \%$ of the patient population on VKA undergoes at least 1 invasive procedure. ${ }^{1}$ The treating physician faces a challenge: a delicate balance must be maintained between the risk of TE and the risk of bleeding in the perioperative anticoagulant management. While arterial TE is associated with a high mortality, or in survivors with major disability, ${ }^{2}$ perioperative bleeding contributes to the need for reoperation, transfusion, prolonged hospitalization, and in some cases death. ${ }^{3}$ In 2012 the American College of Chest Physicians (ACCP) presented the latest guidelines for perioperative management of antithrombotic therapy. ${ }^{1,4}$

In the management of perioperative anticoagulation 3 options can be considered. First, in procedures with a low bleeding risk and the possibility of local hemostatic measures, such as dental extractions, cataract operations and small dermatologic procedures, it is considered a safe choice to continue VKA use. ${ }^{5-8}$ Second, in patients at low risk of TE, ACCP guidelines recommend stopping warfarin administration 5 days before the intervention and restarting warfarin 12-24 hours after the procedure when adequate hemostasis is secured. Finally, in patients at moderate or high TE risk current ACCP guidelines recommend bridging therapy consisting of parenteral administration of LMWH or unfractionated heparin (UFH) in the periprocedural period, combined with interruption of VKA use. A problem that occurs when comparing different studies that assess bridging anticoagulation is the fact that there is no standardized definition of "bridging anticoagulation"; studies assess bridging protocols in which different kinds and doses of $L M W H$, different time points of administration and cessation of VKA and LMWH are advised. ${ }^{2,9-17}$

The evidence evaluating these 3 options is not very strong, ${ }^{4}$ except for VKA continuation in case of procedures with a low bleeding risk. In general, there is paucity in randomized trials assessing different strategies, and in most cases the evidence is based on single armed cohort studies. ${ }^{9,18-21}$ Furthermore, most available studies have small sample sizes and are therefore underpowered to determine whether a certain management strategy is safe (prevention of bleeding) and efficacious (prevention of TE). ${ }^{12,14,16,18,22}$ Recently, more solid evidence is emerging regarding bleeding risk related to different anticoagulant management options in PM/ICD surgery, indicating that the continuation of VKA might provide a safer option than bridging. ${ }^{13,23-26}$

We systematically reviewed studies assessing different peri-interventional management options, compared to bridging therapy. Participants in these studies are all chronic VKA users who undergo surgery. We aimed to assess both the safety and 
efficacy of the different strategies. Furthermore we performed a meta-analysis comparing studies with bridging therapy to warfarin continuation in PM/ICD surgery; pocket hematoma and non-pocket bleeding risk were assessed. For this review we considered 4 treatment comparisons 1) bridging therapy versus periprocedural cessation of VKA 2) bridging therapy versus periprocedural continuation of VKA, 3) low dose LMWH versus high dose LMWH and 4) early, within 24 hours postoperative restart of $\mathrm{LMWH}$ versus late, after 24 hours postoperative restart of LMWH.

\section{Methods}

\section{Study selection}

We included only studies wherein at least 2 periprocedural anticoagulant regimens were assessed, 1 of which could be classified as bridging anticoagulation, while other regimens consist of periprocedural withdrawal of VKA without administration of heparins, periprocedural continuation of VKA without heparin administration or surgery under sub therapeutic INR without heparin administration. We decided to include studies in which data were assessed of patients with different risk categories for a TE (low, moderate, high) and surgical procedures with different bleeding risks (low, high). The primary outcome is objectively confirmed perioperative TE and is defined as any thromboembolism, death caused by TE, composite score of TE. The secondary outcome is perioperative bleeding and is defined as: minor bleeding, major bleeding, and any bleeding. Major bleeding is defined as any bleeding resulting in death, any intracranial bleeding, and any bleeding that leads to transfusion of packed red cells and/or treatment in a hospital, or joint bleeds. All other bleedings, including pocket hematomas in cardiac device surgery are qualified as minor bleedings. Cohort studies, case control studies, cross sectional studies and controlled trials were eligible. Our study protocol is based on the third edition of the Centre for Reviews and Dissemination guidance for undertaking systematic reviews, York, United Kingdom.

\section{Data sources and searches}

We searched MEDLINE (2001-2011, week 24) and EMBASE (2001-2011, week 36) databases. The search strategy can be found in appendix. A further selection was made on title and abstract. The final selection of articles was made after full reading. 


\section{Data extraction and quality assessment}

Data from individual studies were collected on case report forms consisting of 3 sections: study eligibility, checklist of items for data collection, and quality assessment using the Newcastle-Ottawa Scale (NOS) for cohort studies. ${ }^{27}$

\section{Data synthesis and analysis}

We compared baseline characteristics and type of interventional procedure between continued warfarin therapy and bridging groups on the basis of Fisher's exact test and Student's t-test, in order to assess the comparability of these groups with respect to bleeding risk. In our meta-analysis on PM/ICD surgery the random effects model as proposed by DerSimonian and Laird was chosen; individual studies are weighed according to the method proposed by Mantel-Haenszel and expressed as RRs. ${ }^{28}$ RRs and $95 \%$ confidence intervals of each included study and the overall effect were computed. The primary outcome is total bleeding; bridging therapy is compared to warfarin continuation. We tested for heterogeneity with the Breslow-Day test; we used the method proposed by Higgins et al, expressed as $I^{2}$ to measure inconsistency of effects of bridging therapy. ${ }^{29}$ To assess the possibility of bias of the cumulative evidence, publication bias was explored by presenting funnel plots. There were no tests for publication bias carried out, because with the few studies $(n<10)$ included the power of these tests is too low to distinguish chance from real asymmetry. ${ }^{28}$ We conducted sensitivity analyses in a pre-specified way; the intervention effects were examined according to a) the quality assessment of the individual studies (cutoff point 6 stars) and b) we excluded the randomized trial (1) and the non randomized trial (1) from our analyses. For our analyses we used free access Revman 5.1, provided by the Cochrane collaboration.

\section{Results}

Our database search resulted in a total of 878 studies (including duplications), whereof 517 articles were extracted from MEDLINE and 361 from EMBASE. A first selection on title and abstract yielded a total of 35 articles, further restriction after full reading resulted in a set of 17 studies including a subset of 9 regarding PM/ICD surgery. Eight articles were retrieved by hand searching. For a summary of the search see Figure 3.1. All included studies were published in English. For a summary of the included studies see Table 3.1; for a summary of the effect estimates see Table 3.2. 


\section{Chapter 3}

Figure 3.1 Summary of evidence search and selection.

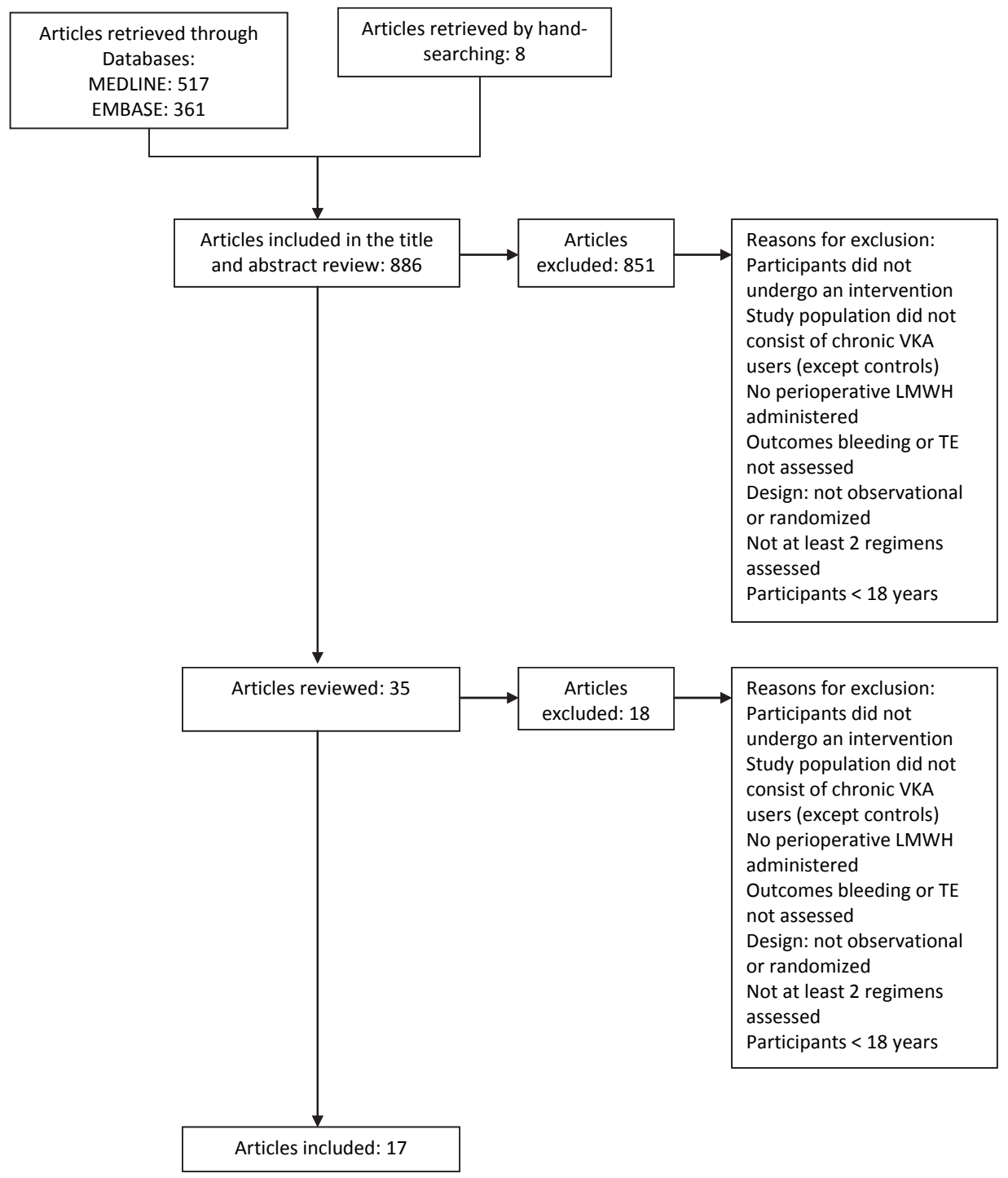

Abbreviations: LMWH, low molecular weight heparins; TE, thromboembolism; VKA, vitamin K antagonists 
Table 3.1 Summary of included studies

\begin{tabular}{|c|c|c|c|c|c|}
\hline Study & Design & Sample size & Determinants & Outcome & Population \\
\hline Ahmed 2010 & $\begin{array}{l}\text { Retrospective } \\
\text { cohort study }\end{array}$ & 459 & $\begin{array}{l}\text { Bridging, warfarin } \\
\text { suspension, } \\
\text { warfarin } \\
\text { continuation }\end{array}$ & $\begin{array}{l}\text { Pocket hematoma, } \\
\text { major bleeding, TE }\end{array}$ & PM/ICD surgery \\
\hline Cano 2010 & $\begin{array}{l}\text { Prospective } \\
\text { cohort study }\end{array}$ & $\begin{array}{l}\text { 849: } 194 \text { on } \\
\text { warfarin }\end{array}$ & $\begin{array}{l}5 \text { groups consisting } \\
\text { of different } \\
\text { antiplatelet and } \\
\text { anticoagulant } \\
\text { regimens and } \\
\text { combinations of } \\
\text { these }\end{array}$ & $\begin{array}{l}\text { Pocket hematoma, } \\
\text { pocket revision, } \\
\text { pericardial effusion, } \\
\text { hemothorax, TE }\end{array}$ & PM/ICD surgery \\
\hline Chow 2010 & $\begin{array}{l}\text { Retrospective } \\
\text { cohort study }\end{array}$ & $\begin{array}{l}\text { 518: } 78 \text { on } \\
\text { warfarin }\end{array}$ & $\begin{array}{l}\text { Bridging, no } \\
\text { bridging }\end{array}$ & $\begin{array}{l}\text { Hematoma, re- } \\
\text { operation/surgical } \\
\text { evacuation }\end{array}$ & $\begin{array}{l}\text { Pacemaker } \\
\text { surgery in elderly }\end{array}$ \\
\hline Daniels 2009 & $\begin{array}{l}\text { Retrospective } \\
\text { cohort study }\end{array}$ & $\begin{array}{l}\text { 556: } 580 \\
\text { procedures }\end{array}$ & $\begin{array}{l}\text { Bridging, warfarin } \\
\text { suspension }\end{array}$ & $\begin{array}{l}\text { Minor bleeding, major } \\
\text { bleeding, TE }\end{array}$ & $\begin{array}{l}\text { Periprocedural } \\
\text { MHV patients }\end{array}$ \\
\hline Garcia 2008 & $\begin{array}{l}\text { Prospective } \\
\text { cohort study }\end{array}$ & $\begin{array}{l}1024 \text { (only } \\
\text { first } \\
\text { procedure) }\end{array}$ & $\begin{array}{l}\text { Bridging, warfarin } \\
\text { suspension }\end{array}$ & $\begin{array}{l}\text { Minor bleeding, major } \\
\text { bleeding, TE }\end{array}$ & $\begin{array}{l}\text { All patients and } \\
\text { AF patients in } \\
\text { minor } \\
\text { procedures }\end{array}$ \\
\hline $\begin{array}{l}\text { Ghanbari } \\
2010\end{array}$ & $\begin{array}{l}\text { Retrospective } \\
\text { cohort study }\end{array}$ & 123 & $\begin{array}{l}\text { Bridging, warfarin } \\
\text { suspension, } \\
\text { warfarin } \\
\text { continuation }\end{array}$ & Pocket hematoma & PM/ICD surgery \\
\hline $\begin{array}{l}\text { Hammerstingl } \\
2009\end{array}$ & $\begin{array}{l}\text { Prospective } \\
\text { cohort study }\end{array}$ & 703 & $\begin{array}{l}\text { Protocol based on } \\
\text { ACCP guidelines } \\
\text { (LMWH versus no } \\
\text { LMWH) }\end{array}$ & $\begin{array}{l}\text { Minor bleeding, major } \\
\text { bleeding overall } \\
\text { bleeding, TE (arterial) }\end{array}$ & $\begin{array}{l}\text { Periprocedural } \\
\text { AF patients }\end{array}$ \\
\hline Jaffer 2010 & $\begin{array}{l}\text { Prospective } \\
\text { cohort study }\end{array}$ & 492 & $\begin{array}{l}\text { No, prophylactic } \\
\text { dose, full dose post } \\
\text { OK LMWH }\end{array}$ & $\begin{array}{l}\text { Minor bleeding, major } \\
\text { bleeding, any } \\
\text { bleeding, TE }\end{array}$ & $\begin{array}{l}\text { Different types } \\
\text { of surgery }\end{array}$ \\
\hline Krane 2008 & $\begin{array}{l}\text { Retrospective } \\
\text { cohort study }\end{array}$ & $\begin{array}{l}238: 57 \text { on } \\
\text { warfarin, } \\
181 \text { controls }\end{array}$ & $\begin{array}{l}\text { Bridging, warfarin } \\
\text { suspension, } \\
\text { controls (no vitamin } \\
\text { K antagonists) }\end{array}$ & $\begin{array}{l}\text { Estimated blood loss, } \\
\text { change of Hgb, } \\
\text { packed cells, catheter } \\
\text { duration, TE }\end{array}$ & $\begin{array}{l}\text { Robotic assisted } \\
\text { radical } \\
\text { prostatectomy }\end{array}$ \\
\hline Li 2011 & $\begin{array}{l}\text { Retrospective } \\
\text { cohort study }\end{array}$ & 766 & $\begin{array}{l}\text { Bridging, warfarin } \\
\text { suspension, } \\
\text { warfarin } \\
\text { continuation }\end{array}$ & $\begin{array}{l}\text { Pocket hematoma, } \\
\text { systemic bleeding, TE }\end{array}$ & PM/ICD surgery \\
\hline Page 2010 & $\begin{array}{l}\text { Non } \\
\text { randomized } \\
\text { controlled trial }\end{array}$ & 198 & $\begin{array}{l}\text { Bridging, warfarin } \\
\text { continuation }\end{array}$ & $\begin{array}{l}\text { Minor bleeding, major } \\
\text { bleeding, overall } \\
\text { bleeding }\end{array}$ & $\begin{array}{l}\text { Catheter } \\
\text { ablation. }\end{array}$ \\
\hline Rhodes 2009 & $\begin{array}{l}\text { Retrospective } \\
\text { cohort study }\end{array}$ & 77 & $\begin{array}{l}\text { Bridging, warfarin } \\
\text { continuation }\end{array}$ & $\begin{array}{l}\text { Complications, packed } \\
\text { cells }\end{array}$ & $\begin{array}{l}\text { Total knee } \\
\text { arthroplasty }\end{array}$ \\
\hline $\begin{array}{l}\text { Robinson } \\
2009\end{array}$ & $\begin{array}{l}\text { Prospective } \\
\text { cohort study }\end{array}$ & 148 & $\begin{array}{l}4 \text { separate bridging } \\
\text { protocols }\end{array}$ & $\begin{array}{l}\text { Hematoma at } \\
\text { pacemaker site, TE }\end{array}$ & PM/ICD surgery \\
\hline
\end{tabular}


Chapter 3

Table 3.1 (continued)

\begin{tabular}{|c|c|c|c|c|c|}
\hline Study & Design & $\begin{array}{l}\text { Sample } \\
\text { size }\end{array}$ & Determinants & Outcome & Population \\
\hline $\begin{array}{l}\text { Tischenko } \\
2009\end{array}$ & $\begin{array}{l}\text { Prospective } \\
\text { cohort study }\end{array}$ & $\begin{array}{l}\text { 272: } 155 \\
\text { on } \\
\text { warfarin }\end{array}$ & $\begin{array}{l}\text { Bridging, warfarin } \\
\text { continuation, controls (no } \\
\text { vitamin K antagonists) }\end{array}$ & $\begin{array}{l}\text { Hematomas, pocket } \\
\text { revision, TE }\end{array}$ & $\begin{array}{l}\text { PM/ICD } \\
\text { surgery }\end{array}$ \\
\hline $\begin{array}{l}\text { Tolosana } \\
2009\end{array}$ & $\begin{array}{l}\text { Randomized } \\
\text { controlled trial }\end{array}$ & 101 & $\begin{array}{l}\text { Bridging, sub therapeutic } \\
\text { INR }\end{array}$ & $\begin{array}{l}\text { Pocket hematoma, } \\
\text { drainage hematoma, } \\
\text { TE }\end{array}$ & $\begin{array}{l}\text { Cardiac } \\
\text { device } \\
\text { surgery }\end{array}$ \\
\hline $\begin{array}{l}\text { Tompkins } \\
2010\end{array}$ & $\begin{array}{l}\text { Retrospective } \\
\text { cohort study }\end{array}$ & $\begin{array}{l}\text { 1388: } 458 \\
\text { on } \\
\text { warfarin }\end{array}$ & $\begin{array}{l}\text { Bridging, INR }>=1.5 \text {, INR }<1.5 \text {, } \\
\text { control group (no vitamin } \mathrm{K} \\
\text { antagonist use) }\end{array}$ & $\begin{array}{l}\text { Pocket exploration, } \\
\text { blood transfusion, } \\
\text { hematoma, TE }\end{array}$ & $\begin{array}{l}\text { PM/ICD } \\
\text { surgery }\end{array}$ \\
\hline $\begin{array}{l}\text { Wazni } \\
2007\end{array}$ & Cohort study & 355 & $\begin{array}{l}\text { Therapeutic dose } \\
\text { enoxaparin, prophylactic. } \\
\text { dose enoxaparin (bridging), } \\
\text { warfarin continuation }\end{array}$ & $\begin{array}{l}\text { Minor bleeding, } \\
\text { major bleeding, } \\
\text { pericardial effusion, } \\
\text { TE }\end{array}$ & AF ablation \\
\hline
\end{tabular}

Abbreviations: ACCP, American Congress of Chest Physicians; AF, atrial fibrillation ; Hgb, Hemoglobin ; INR, International Normalized Ratio; LMWH, Low Molecular Weight Heparin; MHV, mechanical heart valves; $\mathrm{PM} / \mathrm{ICD}$, pacemaker/implantable cardioverter defibrillator; $\mathrm{TE}$, thromboembolism

Table 3.2 Summary of the effect estimates of the included studies

\begin{tabular}{|c|c|c|c|}
\hline Study & Comparison & Outcome & $\begin{array}{l}\text { Effect estimate }(95 \% \mathrm{Cl}) \text { or } \mathrm{p} \text { - } \\
\text { value }\end{array}$ \\
\hline Ahmed 2010 & Bridging-warfarin continuation & Pocket hematoma & $\begin{array}{l}\mathrm{p}=0.004 ; \text { significantly more } \\
\text { pocket hematomas in bridging } \\
\text { group }\end{array}$ \\
\hline Cano 2010 & $\begin{array}{l}5 \text { groups consisting of different } \\
\text { antiplatelet and anticoagulant } \\
\text { regimens and combinations }\end{array}$ & Pocket hematoma & $\begin{array}{l}\text { OR enoxaparin bridging: } 7.10 \\
(95 \% \mathrm{Cl}, 3.34-15.09)\end{array}$ \\
\hline Chow 2010 & Bridging-warfarin continuation & Pocket hematoma & $\begin{array}{l}p<0.001 \text {;significantly more } \\
\text { pocket hematomas in bridging } \\
\text { group }\end{array}$ \\
\hline Daniels 2009 & $\begin{array}{l}\text { Bridging with LMWH, bridging } \\
\text { with UFH, warfarin stop }\end{array}$ & Major bleeding & $\begin{array}{l}p=0.26 \text {; no significant } \\
\text { differences between groups }\end{array}$ \\
\hline Garcia 2008 & Bridging-warfarin cessation & $\begin{array}{l}\text { Clinically significant } \\
\text { bleeding }\end{array}$ & N.R. \\
\hline Ghanbari 2010 & $\begin{array}{l}\text { Bridging, warfarin suspension, } \\
\text { warfarin continuation }\end{array}$ & Pocket hematoma & $\begin{array}{l}p=0.03 \text {; significantly more } \\
\text { pocket hematomas in bridging } \\
\text { group }\end{array}$ \\
\hline $\begin{array}{l}\text { Hammerstingl } \\
2009\end{array}$ & $\begin{array}{l}\text { Protocol based on ACCP } \\
\text { guidelines }\end{array}$ & Bleeding events & $\begin{array}{l}p=0.004 \text {; total enoxaparin dose } \\
\text { significantly increased bleeding } \\
\text { risk }\end{array}$ \\
\hline Jaffer 2010 & $\begin{array}{l}\text { No dose, prophylactic dose, full } \\
\text { dose post OK LMWH }\end{array}$ & Bleeding events & $\begin{array}{l}\text { OR full dose heparin: } 5.1 \text { (95\% } \\
\mathrm{Cl}, 2.3-11.6)\end{array}$ \\
\hline Krane 2008 & Bridging-warfarin cessation & Risk of transfusion & $\begin{array}{l}p=0.04 ; \text { significantly higher risk } \\
\text { of transfusion in bridging group }\end{array}$ \\
\hline Li 2011 & $\begin{array}{l}\text { Bridging, warfarin suspension, } \\
\text { warfarin continuation }\end{array}$ & Bleeding events & $\begin{array}{l}p=0.03 \text {; significantly more } \\
\text { bleedings in bridging group }\end{array}$ \\
\hline
\end{tabular}


Table 3.2 (continued)

\begin{tabular}{|c|c|c|c|}
\hline Study & Comparison & Outcome & $\begin{array}{l}\text { Effect estimate }(95 \% \mathrm{Cl}) \text { or } \mathrm{p} \text { - } \\
\text { value }\end{array}$ \\
\hline Page 2010 & Bridging-warfarin continuation & Bleeding events & $\begin{array}{l}\mathrm{p}<0.001 ; \text { significantly more } \\
\text { bleedings in bridging group }\end{array}$ \\
\hline Rhodes 2009 & Warfarin continuation-bridging & Risk of transfusion & RR: 0.61 (95\% Cl, 0.20-1.86); \\
\hline Robinson 2009 & 4 separate bridging protocols & Pocket hematoma & $\begin{array}{l}\mathrm{p}<0.001 \text {; significantly more } \\
\text { pocket hematomas in } \\
\text { postoperative LMWH group }\end{array}$ \\
\hline Tischenko 2009 & $\begin{array}{l}\text { Bridging-warfarin continuation- } \\
\text { controls }\end{array}$ & Pocket revision & $\begin{array}{l}\mathrm{p}=0.46 \text {; warfarin continuation } \\
\text { compared to bridging }\end{array}$ \\
\hline Tolosana 2009 & Bridging-warfarin continuation & Pocket hematoma & $p=1.00$ \\
\hline Tompkins 2010 & $\begin{array}{l}\text { Bridging-warfarin continuation- } \\
\text { controls }\end{array}$ & Bleeding events & $\begin{array}{l}\text { OR for heparin use: } 9.88 \text { (95\% } \\
\mathrm{Cl}, 3.16-30.91)\end{array}$ \\
\hline Wazni 2007 & $\begin{array}{l}\text { Therapeutic dose enoxaparin, } \\
\text { prophylactic. dose enoxaparin } \\
\text { (bridging), warfarin } \\
\text { continuation }\end{array}$ & Major bleeding & $\begin{array}{l}p<0.001 \text {; significantly more } \\
\text { bleedings in therapeutic dose } \\
\text { enoxaparin group }\end{array}$ \\
\hline
\end{tabular}

Abbreviations: ACCP, American Congress of Chest Physicians; $\mathrm{Cl}$, confidence interval; LMWH, Low Molecular Weight Heparin; OR, odds ratio; RR, relative risk; UFH, unfractionated heparin

The main concern about the internal validity of the included observational studies is the fact that the differences between the comparator groups are not corrected for in the majority of the studies, which probably influences the risk estimates of the individual studies substantially. ${ }^{11,23,26,30,31}$ The most important discrepancy between study groups lies in the fact that the ratio of low, intermediate and high TE risk patients differs significantly in the comparator groups, due to the observational design of the studies; patients at high risk of a TE tend to be bridged differently than moderate or low TE risk patients. In Table 3.3 a summary of potential sources of bias in the individual studies is given. NOS scores are reported in the last column. No study was awarded with the maximum of 9 stars. The considerations mentioned above, justify the decision to confine our meta-analysis to studies containing predominantly high TE risk patients undergoing the same procedure, namely PM/ICD surgery. 
Table 3.3 Summary of potential sources of bias in individual studies

\begin{tabular}{|c|c|c|c|c|c|c|c|}
\hline Study & $\begin{array}{l}\text { Selection of } \\
\text { non- } \\
\text { exposed } \\
\text { cohort }\end{array}$ & $\begin{array}{l}\text { Ascertainment } \\
\text { of exposure }\end{array}$ & $\begin{array}{l}\text { Comparability } \\
\text { cohorts, } \\
\text { adjustments for } \\
\text { differences }\end{array}$ & $\begin{array}{l}\text { Ascertainment } \\
\text { of outcome }\end{array}$ & $\begin{array}{l}\text { Follow } \\
\text { up } \\
\text { period }\end{array}$ & $\begin{array}{l}\text { Lost to } \\
\text { follow up }\end{array}$ & $\begin{array}{l}\text { NOS } \\
\text { score }\end{array}$ \\
\hline $\begin{array}{l}\text { Ahmed } \\
2010\end{array}$ & $\begin{array}{l}\text { Same } \\
\text { community } \\
\text { as exposed } \\
\text { cohort }\end{array}$ & Patient records & $\begin{array}{l}\text { Lower aspirin use } \\
\text { in warfarin } \\
\text { continuation } \\
\text { group, differences } \\
\text { in indications VKA } \\
\text { therapy; Fischer's } \\
\text { exact test }\end{array}$ & Record linkage & 8 weeks & $\begin{array}{l}\text { All subjects } \\
\text { accounted } \\
\text { for }\end{array}$ & 6 \\
\hline Cano 2010 & $\begin{array}{l}\text { Same } \\
\text { community } \\
\text { as exposed } \\
\text { cohort }\end{array}$ & Patient records & $\begin{array}{l}\text { No significant } \\
\text { differences } \\
\text { reported between } \\
\text { study groups; } \\
\text { multivariable } \\
\text { adjusted analyses }\end{array}$ & Record linkage & 7 days & $\begin{array}{l}\text { All subjects } \\
\text { accounted } \\
\text { for }\end{array}$ & 5 \\
\hline Chow 2010 & $\begin{array}{l}\text { Same } \\
\text { community } \\
\text { as exposed } \\
\text { cohort }\end{array}$ & Patient records & $\begin{array}{l}\text { No differences } \\
\text { between study } \\
\text { groups reported; } \\
\text { multivariable } \\
\text { adjusted analyses }\end{array}$ & Record linkage & 6 weeks & $\begin{array}{l}12 / 518 \text { lost } \\
\text { to follow } \\
\text { up }\end{array}$ & 6 \\
\hline $\begin{array}{l}\text { Daniels } \\
2009\end{array}$ & $\begin{array}{l}\text { Same } \\
\text { community } \\
\text { as exposed } \\
\text { cohort }\end{array}$ & $\begin{array}{l}\text { Patient } \\
\text { records, } \\
\text { interviews }\end{array}$ & $\begin{array}{l}\text { Bridging group } \\
\text { moderate/high TE } \\
\text { risk, warfarin } \\
\text { cessation group } \\
\text { low TE risk } \\
\text { participants; } \\
\text { univariable } \\
\text { analyses }\end{array}$ & Record linkage & 90 days & $\begin{array}{l}\text { All subjects } \\
\text { accounted } \\
\text { for }\end{array}$ & 6 \\
\hline $\begin{array}{l}\text { Garcia } \\
2008\end{array}$ & $\begin{array}{l}\text { Same } \\
\text { community } \\
\text { as exposed } \\
\text { cohort }\end{array}$ & Patient records & $\begin{array}{l}\text { No differences } \\
\text { reported between } \\
\text { study groups; } \\
\text { univariable } \\
\text { analyses }\end{array}$ & Record linkage & 30 days & $\begin{array}{l}1 / 1024 \text { lost } \\
\text { to follow } \\
\text { up }\end{array}$ & 6 \\
\hline $\begin{array}{l}\text { Ghanbari } \\
2010\end{array}$ & $\begin{array}{l}\text { Same } \\
\text { community } \\
\text { as exposed } \\
\text { cohort }\end{array}$ & Patient records & $\begin{array}{l}\text { Warfarin } \\
\text { continuation } \\
\text { group and } \\
\text { bridging group: } \\
\text { only high TE risk } \\
\text { participants, } \\
\text { warfarin cessation } \\
\text { group low TE risk; } \\
\text { multivariable } \\
\text { adjusted analyses }\end{array}$ & Record linkage & NR & $\begin{array}{l}\text { All subjects } \\
\text { accounted } \\
\text { for }\end{array}$ & 5 \\
\hline
\end{tabular}


Table 3.3 (continued)

\begin{tabular}{|c|c|c|c|c|c|c|c|}
\hline Study & $\begin{array}{l}\text { Selection of } \\
\text { non- } \\
\text { exposed } \\
\text { cohort }\end{array}$ & $\begin{array}{l}\text { Ascertainment } \\
\text { of exposure }\end{array}$ & $\begin{array}{l}\text { Comparability } \\
\text { cohorts, } \\
\text { adjustments for } \\
\text { differences }\end{array}$ & $\begin{array}{l}\text { Ascertainment } \\
\text { of outcome }\end{array}$ & $\begin{array}{l}\text { Follow } \\
\text { up } \\
\text { period }\end{array}$ & $\begin{array}{l}\text { Lost to } \\
\text { follow up }\end{array}$ & $\begin{array}{l}\text { NOS } \\
\text { score }\end{array}$ \\
\hline $\begin{array}{l}\text { Hammersti } \\
\text { ngl } 2009\end{array}$ & $\begin{array}{l}\text { Same } \\
\text { community } \\
\text { as exposed } \\
\text { cohort }\end{array}$ & Patient records & $\begin{array}{l}\text { No differences } \\
\text { reported between } \\
\text { groups; } \\
\text { univariable } \\
\text { analyses, } \\
\text { multivariable } \\
\text { analyses }\end{array}$ & Record linkage & 30 days & $\begin{array}{l}\text { All subjects } \\
\text { accounted } \\
\text { for }\end{array}$ & 8 \\
\hline Jaffer 2010 & $\begin{array}{l}\text { Same } \\
\text { community } \\
\text { as exposed } \\
\text { cohort }\end{array}$ & Patient records & $\begin{array}{l}\text { No significant } \\
\text { differences } \\
\text { reported between } \\
\text { study groups; } \\
\text { multivariable } \\
\text { adjusted analyses }\end{array}$ & Record linkage & NR & $\begin{array}{l}\text { All subjects } \\
\text { accounted } \\
\text { for }\end{array}$ & 8 \\
\hline Krane 2008 & $\begin{array}{l}\text { Same } \\
\text { community } \\
\text { as exposed } \\
\text { cohort }\end{array}$ & Patient records & $\begin{array}{l}\text { No significant } \\
\text { differences } \\
\text { reported between } \\
\text { study groups; } \\
\text { univariable } \\
\text { analyses }\end{array}$ & Record linkage & NR & $\begin{array}{l}3 / 60 \text { lost to } \\
\text { follow up }\end{array}$ & 4 \\
\hline Li 2011 & $\begin{array}{l}\text { Same } \\
\text { community } \\
\text { as exposed } \\
\text { cohort }\end{array}$ & Patient records & $\begin{array}{l}\text { Study groups } \\
\text { differ in age, \% } \\
\text { high TE risk, } \\
\text { indication } \\
\text { warfarin; } \\
\text { multivariable } \\
\text { adjusted analyses, } \\
\text { subgroup analyses } \\
\text { high TE risk } \\
\text { participants }\end{array}$ & $\begin{array}{l}\text { Transtelephoni } \\
\text { c evaluation }\end{array}$ & 4 weeks & $\begin{array}{l}\text { All subjects } \\
\text { accounted } \\
\text { for }\end{array}$ & 7 \\
\hline Page 2010 & $\begin{array}{l}\text { Same } \\
\text { community } \\
\text { as exposed } \\
\text { cohort }\end{array}$ & Patient records & $\begin{array}{l}\text { \% Hypertension, } \\
\text { serum creatinine } \\
\text { level differ } \\
\text { between groups; } \\
\text { univariable } \\
\text { analyses }\end{array}$ & $\begin{array}{l}\text { Medical } \\
\text { examination } \\
\text { day }+1+ \\
\text { telephone } \\
\text { questionnaire }\end{array}$ & $\begin{array}{l}4 \text { to } 6 \\
\text { weeks }\end{array}$ & $\begin{array}{l}\text { All subjects } \\
\text { accounted } \\
\text { for }\end{array}$ & 5 \\
\hline $\begin{array}{l}\text { Rhodes } \\
2009\end{array}$ & $\begin{array}{l}\text { Same } \\
\text { community } \\
\text { as exposed } \\
\text { cohort }\end{array}$ & Patient records & $\begin{array}{l}\text { Age, diagnosis for } \\
\text { Coumadin differ } \\
\text { between groups; } \\
\text { multivariable } \\
\text { adjusted analyses }\end{array}$ & Record linkage & 90 days & $\begin{array}{l}\text { All subjects } \\
\text { accounted } \\
\text { for }\end{array}$ & 7 \\
\hline $\begin{array}{l}\text { Robinson } \\
2009\end{array}$ & $\begin{array}{l}\text { Same } \\
\text { community } \\
\text { as exposed } \\
\text { cohort; } \\
\text { subsequent } \\
\text { protocols }\end{array}$ & Patient records & $\begin{array}{l}\text { No significant } \\
\text { differences } \\
\text { reported between } \\
\text { study groups; } \\
\text { multivariable } \\
\text { adjusted analyses }\end{array}$ & Record linkage & $\begin{array}{l}1-4 \\
\text { weeks }\end{array}$ & $\begin{array}{l}\text { All subjects } \\
\text { accounted } \\
\text { for }\end{array}$ & 8 \\
\hline
\end{tabular}


Table 3.3 (continued)

\begin{tabular}{|c|c|c|c|c|c|c|c|}
\hline Study & $\begin{array}{l}\text { Selection of } \\
\text { non- } \\
\text { exposed } \\
\text { cohort }\end{array}$ & $\begin{array}{l}\text { Ascertainment } \\
\text { of exposure }\end{array}$ & $\begin{array}{l}\text { Comparability } \\
\text { cohorts, } \\
\text { adjustments for } \\
\text { differences }\end{array}$ & $\begin{array}{l}\text { Ascertainment } \\
\text { of outcome }\end{array}$ & $\begin{array}{l}\text { Follow } \\
\text { up } \\
\text { period }\end{array}$ & $\begin{array}{l}\text { Lost to } \\
\text { follow up }\end{array}$ & $\begin{array}{l}\text { NOS } \\
\text { score }\end{array}$ \\
\hline $\begin{array}{l}\text { Tischenko } \\
2009\end{array}$ & $\begin{array}{l}\text { Same } \\
\text { community } \\
\text { as exposed } \\
\text { cohort }\end{array}$ & Patient records & $\begin{array}{l}\text { No significant } \\
\text { differences } \\
\text { reported between } \\
\text { study groups, only } \\
\text { high TE risk } \\
\text { participants in } \\
\text { warfarin } \\
\text { continuation } \\
\text { group and } \\
\text { bridging group; } \\
\text { univariable } \\
\text { analyses }\end{array}$ & Record linkage & 1 week & $\begin{array}{l}272 / 447 \\
\text { lost to } \\
\text { follow up, } \\
\text { no } \\
\text { description } \\
\text { given }\end{array}$ & 4 \\
\hline $\begin{array}{l}\text { Tolosana } \\
2009\end{array}$ & $\begin{array}{l}\text { Randomized } \\
\text { allocation }\end{array}$ & Patient records & $\begin{array}{l}\text { Only high TE risk } \\
\text { participants; } \\
\text { univariable } \\
\text { analyses }\end{array}$ & Record linkage & 45 days & $\begin{array}{l}\text { All subjects } \\
\text { accounted } \\
\text { for }\end{array}$ & 8 \\
\hline $\begin{array}{l}\text { Tompkins } \\
2010\end{array}$ & $\begin{array}{l}\text { Same } \\
\text { community } \\
\text { as exposed } \\
\text { cohort }\end{array}$ & Patient records & $\begin{array}{l}\text { No significant } \\
\text { differences } \\
\text { reported between } \\
\text { study groups; } \\
\text { multivariable } \\
\text { analyses }\end{array}$ & Record linkage & 5 days & $\begin{array}{l}\text { All subjects } \\
\text { accounted } \\
\text { for }\end{array}$ & 8 \\
\hline $\begin{array}{l}\text { Wazni } \\
2007\end{array}$ & $\begin{array}{l}\text { Same } \\
\text { community } \\
\text { as exposed } \\
\text { cohort }\end{array}$ & Patient records & $\begin{array}{l}\text { No differences } \\
\text { reported between } \\
\text { study groups; } \\
\text { univariable } \\
\text { analyses }\end{array}$ & $\begin{array}{l}\text { Medical } \\
\text { examination } \\
\text { day }+1 \text {, self } \\
\text { reporting }\end{array}$ & NR & $\begin{array}{l}\text { No } \\
\text { statement }\end{array}$ & 4 \\
\hline
\end{tabular}

Abbreviations: NOS, Newcastle-Ottawa scale; NR, not reported; TE, thromboembolism; VKA, vitamin K antagonists

\section{Perioperative anticoagulation strategies}

In the articles that met our inclusion criteria, we identified 3 strategies used for the management of perioperative anticoagulation: i) bridging anticoagulation in prophylactic or therapeutic doses with LMWH or UFH; ii) the interruption of VKA without bridging therapy; iii) The continuation of VKA with therapeutic or sub therapeutic INR. These 3 comparisons are discussed successively.

\section{Thrombotic events}

The primary outcome TE in patients on chronic anticoagulation was assessed in 13 of the 17 included studies. Percentages of the outcome in different studies range from zero (4) to $2.6(1) .{ }^{9,10,12,13,24,32}$ None of the included studies reported significant 
differences in cumulative incidence of TEs with regard to the comparisons made in this review. For the meta-analysis of bridging therapy compared to VKA continuation in PM/ICD surgery; only 3 studies reported on TE and could therefore not be included in these analyses, this number would be too small to yield relevant results.

\section{Bridging therapy and interruption of anticoagulants}

Bridging therapy consisting of different LMWH or UFH in both prophylactic and therapeutic doses was compared to cessation of VKA in 8 studies. ${ }^{11,23-25,30,32-34}$ All of these studies had an observational design. In only 2 of these studies authors reported that in patients in whom warfarin was interrupted TE risk was predominantly low and when bridging was applied patients had mainly moderate to high TE risk. ${ }^{11,25}$ This is in accordance with the recommendation by the current ACCP guidelines. The other authors did not specify differences in TE risk. The type of procedures differed between the studies (Table 3.1). All outcomes of interest could be classified as TE, minor bleeding, major bleeding or overall bleeding. The study by Krane et al, mentioned an increased risk of transfusion for bridged patients after robotic-assisted radical prostatectomy compared to interrupted VKA patients $(p=0.04)$; this analysis was unadjusted. $^{33}$ In a retrospective cohort study performed by Daniels et al. the comparison of LMWH or UFH to no heparin in patients with mechanical heart valves undergoing different types of surgery resulted in an unadjusted RR of $1.25(95 \% \mathrm{Cl}$, $0.73-2.14)$ for overall bleeding. ${ }^{11}$ Pooling the results of 7 individual studies for the outcome minor bleeding resulted as expected in heterogeneity $(p<0.001$; $\mathrm{I}^{2}=78 \%$ ). ${ }^{11,16,23-25,30,34}$ Analysis of major bleeding, overall bleeding and TE resulted in heterogeneity as well (results not shown). In 3 studies multivariable adjusted analyses were presented. First, a study performed by Cano et al. reported an odds ratio (OR) of 7.10 (95\% Cl, 3.34-15.09) for pocket hematoma in patients bridged with enoxaparin who underwent the implantation of cardiac rhythm devices compared to no enoxaparin administration. ${ }^{32}$ Secondly, Tompkins et al. reported an OR for heparin use compared to no heparin use of $9.88(95 \% \mathrm{Cl}, 3.16-30.91)$ for bleeding complications in patients after cardiac device implantation. ${ }^{23}$ Thirdly, Li et al. computed, after adjusting for age and gender a p-value of 0.02 comparing warfarin cessation to bridging in $\mathrm{PM} / \mathrm{ICD}$ surgery; the incidence of overall bleeding was higher in the bridging group. ${ }^{24}$ Ghanbari et al. reported in a study on PM/ICD surgery an unadjusted OR of 6.17 (95\% $\mathrm{Cl}, 1.6-24)$ for major device pocket hematoma in a high TE risk group on bridging therapy. ${ }^{25}$ Finally, Garcia et al. and Ahmed et al. reported no significant differences in bleeding risk in their studies of patients undergoing minor procedures and PM/ICD surgery, respectively. ${ }^{30,34}$ 


\section{Bridging therapy and continuation of warfarin}

Bridging therapy consisting of different LMWH and UFH in both prophylactic and therapeutic doses was compared to continuation of VKA in 9 studies ${ }^{12-14,23-26,31,34}$ whereof 6 studies regarding PM/ICD interventions. ${ }^{12,13,23-25,34}$ The 3 remaining studies assessed the comparison in catheter ablation (2) and total knee arthroplasty (TKA) (1). These 9 studies included 7 cohort studies, ${ }^{13,14,23-25,31,34} 1$ randomized trial ${ }^{12}$ and 1 non randomized trial. ${ }^{26}$ See Table 3.1 for a summary of the included studies. All outcomes of interest could be classified as TE, minor bleeding, major bleeding or overall bleeding. Again, pooling of these studies resulted in unacceptable heterogeneity $\left(p<0.001 ; l^{2}=76 \%\right)$.

Rhodes et al. presented an age adjusted RR of $0.61(95 \% \mathrm{Cl}, 0.20-1.86)$ for transfusion required, when they compared a group that continued coumadin with a bridging group in TKA surgery. ${ }^{14} \mathrm{~A}$ non randomized trial including patients undergoing a catheter ablation performed by Page et al. resulted in overall bleeding in the bridging LMWH group of $78 \%$ and $56 \%$ in the warfarin group $(p<0.001) .{ }^{26}$ A randomized controlled trial by Tolosana et al. resulted in $p$ values of 1.00 for both pocket hematoma and drainage hematoma. ${ }^{12}$

We performed a meta-analysis assessing the risk of minor bleeding (pocket hematoma), major bleeding (pocket revision, drainage hematoma, pericardial tamponnade and hemothorax) and overall bleeding in PM/ICD surgery. We included 6 studies with an observational design $(5)^{13,23-25,34}$ and randomized allocation (1), ${ }^{12}$ all with unadjusted results. In all studies, except the study performed by Tolosana, the patients on warfarin had a therapeutic INR. The studies performed by Ghanbari, Tischenko and Tolosana included only high TE risk patients. For the outcome minor bleeding we were able to use a subgroup of patients from the study of Li et al, with high TE risk. ${ }^{24}$ Differences with respect to known risk factors for bleeding (age, male sex, diabetes, concomitant antiplatelet drug use, bleeding disorders, kind and extend of procedure) were assessed between the bridging and continued warfarin group using Fisher's exact test and the Student's t-test. ${ }^{9,35,36}$ Significant differences between these groups are higher proportions of aspirin use in the bridging group in the study performed by $\mathrm{Li}^{24}$ higher proportions of patients with diabetes in the bridging group in the study by Ahmed et al. ${ }^{34}$ and younger age in the bridging group in studies by $\mathrm{Li}^{24}$ and Tischenko et al. ${ }^{13}$ (results not shown).

Homogenous results $\left(p=0.29, I^{2}=19 \%\right)$ were observed regarding pocket hematoma, and a RR of 2.62 (95\% Cl, 1.41-4.86) was computed. For major bleeding we observed homogenous results too $\left(p=0.36,1^{2}=8 \%\right)$ with a borderline insignificant $R R$ of 3.26 $(95 \% \mathrm{Cl}, 0.99-10.78)$. Analyses regarding overall bleeding resulted in a homogenous 
result $\left(p=0.31, I^{2}=16 \%\right.$ ) with a $R R$ of $3.03(95 \% \mathrm{Cl}, 1.86-4.95)$. In Figure 3.2 the forest plot is presented; Figure 3.3 depicts the funnel plot, wherein the effect sizes of the studies are plotted against the study precision. Excluding the only RCT from our analyses resulted in a homogenous $\mathrm{RR}$ of 3.55 (95\% Cl, 2.88-5.63). The exclusion of methodological poor studies, less than 7 points on the NOS scale, left us with 3 studies and resulted in a homogenous $\mathrm{RR}$ of $2.25(95 \% \mathrm{Cl}, 1.12-4.51)$.

Figure 3.2 Forest plot comparison: bridging versus warfarin continuation outcome: overall bleeding. M-H indicates Mantel-Haenszel, $\mathrm{Cl}$ confidence interval.

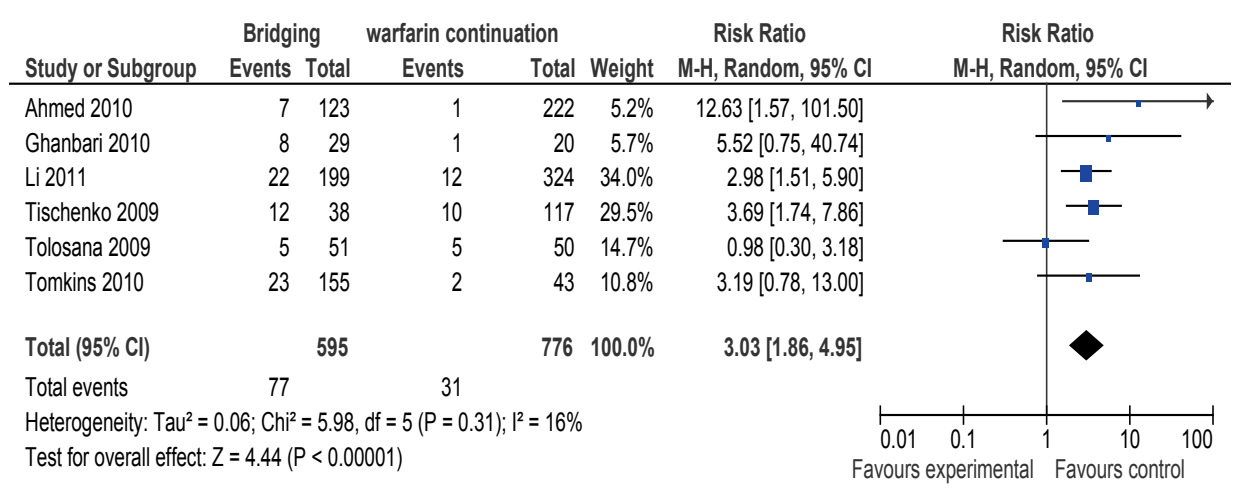

\section{The dose and timing of heparin administration}

Of the included studies, 4 assessed the dosage or timing of heparin administration within the context of bridging therapy. ${ }^{3,9,10,16}$ Jaffer et al. performed 'propensity score' adjusted analyses in a prospective cohort and observed that patients in different kinds of surgery receiving the full dose of heparin had significantly higher odds of any bleeding and major bleeding than those receiving the prophylactic dose or no bridging: an OR of $5.1(95 \% \mathrm{Cl}, 2.3-11.6)$ and 4.1 (95\% $\mathrm{Cl}, 1.4-12.1)$ respectively. ${ }^{3}$ The propensity score is the predicted likelihood that the patient would receive the full dose of heparin. ${ }^{3}$ Also adjusting for medical and surgical bleeding class did not influence the ORs remarkably. Hammerstingl et al. calculated in their prospective cohort study a multivariable adjusted hazard ratio of $1.058(95 \% \mathrm{Cl}, 1.017-1.101)$ per enoxaparin dosage ( $\mathrm{mg} / \mathrm{kg}$ body weight) for bleeding events. ${ }^{9}$ The possible association between timing of heparin administration and bleeding was assessed by Chow et al, concluding after an unadjusted analysis of a retrospective cohort that the time at which heparin was resumed influenced the incidence of postoperative hematoma 
formation in PM surgery (15/21 within 24 hours versus 6/21 after 24 hours: $p<0.001$ ) [16]. Robinson reported in a multivariable adjusted analysis that any postoperative administered LMWH is a risk factor for wound hematoma $(p<0.001) .{ }^{10}$

Figure 3.3 Funnel plot comparison: bridging versus warfarin continuation outcome: overall bleeding. RR indicates relative risk, SE standard error

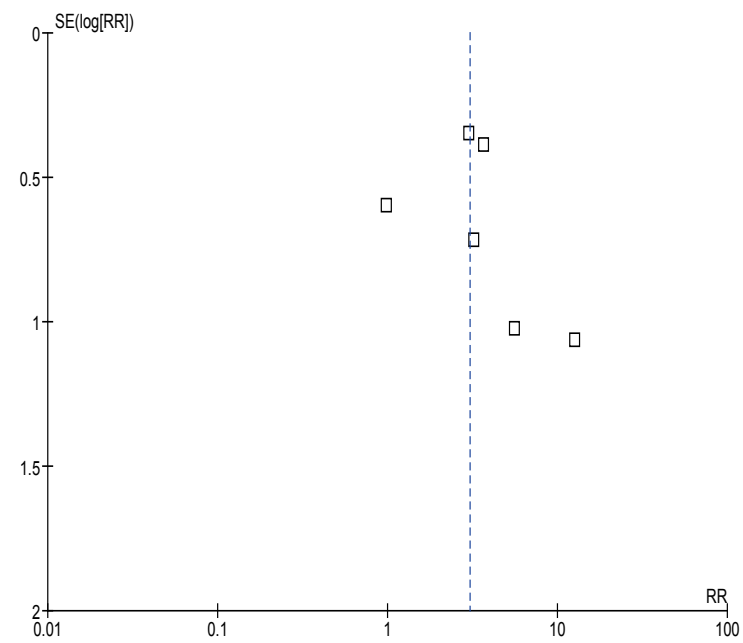

\section{Discussion}

We systematically reviewed the efficacy and safety of bridging therapy in different procedures. The most important finding of this review is that no conclusion can be drawn regarding the efficacy of bridging therapy; in none of the included studies significant differences were reported in the incidence of TEs between different study groups. Increased bleeding risk is observed in different types of surgery, attributed to bridging anticoagulation. However, effect modification of bridging therapy by the type of surgery is probable; therefore we cannot generalize the latter finding.

We detected heterogeneity between studies due to the fact that different procedures, study designs, and populations were assessed. We therefore were not able to provide pooled risk estimates for most studies, we were only able to report a pooled risk estimate of $\mathrm{PM} / \mathrm{ICD}$ procedures. In this review recent studies regarding $\mathrm{PM} / \mathrm{ICD}$ procedures seem somewhat overrepresented, probably due to recently increased attention to the option of continuation of VKA in these low bleeding risk procedures. 
In PM/ICD surgery bridging anticoagulation results in a higher bleeding risk compared to perioperative continuation of VKA. This finding is novel. To our knowledge this is the only review in which a pooled RR is presented for the comparison of bridging therapy to continuation of VKA in PM/ICD surgery.

Most of the 17 included studies that assessed bleeding risk produced biased results. In 4 out of 5 high quality studies (a minimum of 8 stars on the NOS scale) bridging was identified as a risk factor for bleeding ${ }^{3,9,10,23}$; only 1 high quality study reported a null net result. ${ }^{12}$ The finding that bridging is a risk factor for perioperative bleeding is supported by 2 reviews regarding low bleeding risk procedures. ${ }^{37,38}$

The short exposure time to bridging anticoagulation, according to ACCP guidelines 7 to 11 days, might have influenced the detection that no significant differences occurred in incidence of TEs between different study groups; overall TE incidence was low, in some studies zero. Similar findings were reported in other reviews. ${ }^{39,40}$ The net clinical benefit of bridging is therefore unclear. Nevertheless, the risk of a major bleeding can be weighed against the risk of TE, when case-fatality rates are compared. The case-fatality rate for major bleeding is $8-9 \%$. For arterial TE these rates vary from $15 \%$ (heart valve thrombosis) to $70 \%$ (stroke), while venous TE carries a $6 \%$ rate for death or major disability and a $25 \%$ risk for pulmonary embolism. ${ }^{41}$ These numbers suggest that one is more likely to accept major bleedings due to periprocedural anticoagulant strategies to prevent arterial TE. ${ }^{41}$

A possible explanation for the bleeding risk associated with bridging therapy is provided by Gerotziafas et al, who demonstrated that treatment with a combination of VKA and LMWH induces a more profound inhibition of thrombin generation than treatment with VKA alone. ${ }^{42}$ VKA and LMWH administration is usually resumed within 12-78 hours after surgery; LMWH administration is stopped when the INR reaches a value of 2 . In this way a period in which the patient might be more vulnerable for a bleeding is established by the early introduction of bridging therapy. Douketis et al. demonstrated that bridging anticoagulation with LMWH after interruption of warfarin therapy is associated with a residual anticoagulant effect prior to surgery, which might lead to a higher bleeding tendency. ${ }^{43}$

In our meta-analysis on PM/ICD surgery bridging is a risk factor for bleeding. However, these analyses should be interpreted with caution, because in none of these, mostly observational studies, adjusted analyses were presented, and only 2 studies scored 8 points or more on the NOS scale. An earlier meta-analysis on a similar topic performed by Jamula et al. reported that a strategy of warfarin continuation appears to confer approximately one-half the risk (OR: $0.43 ; 95 \% \mathrm{Cl}, 0.26-0.73$ ) of experiencing an access site bleeding complication compared with a strategy of warfarin cessation or bridging in elective coronary angiography with or without $\mathrm{PCl}{ }^{44}$ 
The main limitations of this study are the fact that most of the included studies are of poor methodological quality. Furthermore most of our analyses yielded heterogeneous results; hence only 1 pooled risk estimate could be presented.

Based on previous research, as well as on conclusions presented in this article, the question whether the application of fixed dose LMWH bridging therapy, which may lead to increased risk of bleeding in certain patients is always warranted, seems legitimate. Results provided by large randomized trials like the BRIDGE trial, due in 2013, and the BRUISE control trial in PM/ICD surgery will need to shine more light on the safety and efficacy of bridging. Possibly, a more personalized bridging scheme, including not only TE risk and bleeding risk, but also biomarkers including the individual thrombin generating potential may lead to a better safety and efficacy profile of this intervention. On the other hand, the expected large-scale use of new oral anticoagulants like direct thrombin and factor Xa inhibitors with short half-lives raises the question whether there will be a need to bridge patients who use these new agents. 


\section{References}

1. Douketis JD, Berger PB, Dunn AS, et al. The perioperative management of antithrombotic therapy: American College of Chest Physicians Evidence-Based Clinical Practice Guidelines (8th Edition). Chest 2008;133:299-339.

2. McBane RD, Wysokinski WE, Daniels $P R$, et al. Periprocedural anticoagulation management of patients with venous thromboembolism. Arterioscler Thromb Vasc Biol 2010;30:442-8.

3. Jaffer AK, Brotman DJ, Bash LD, Mahmood SK, Lott B, White RH. Variations in perioperative warfarin management: outcomes and practice patterns at nine hospitals. Am J Med 2010;123:141-50.

4. Douketis JD, Spyropoulos AC, Spencer FA, et al. Perioperative Management of Antithrombotic Therapy: Antithrombotic Therapy and Prevention of Thrombosis, 9th ed: American College of Chest Physicians Evidence-Based Clinical Practice Guidelines. Chest. 2012;141:326-50.

5. Bajkin BV, Popovic SL, Selakovic SD. Randomized, prospective trial comparing bridging therapy using low-molecular-weight heparin with maintenance of oral anticoagulation during extraction of teeth. J Oral Maxillofac Surg 2009;67:990-5.

6. Sacco R, Sacco M, Carpenedo M, Moia M. Oral surgery in patients on oral anticoagulant therapy: a randomized comparison of different INR targets. J Thromb Haemost 2006;4:688-9.

7. Syed S, Adams BB, Liao W, Pipitone M, Gloster H. A prospective assessment of bleeding and international normalized ratio in warfarin-anticoagulated patients having cutaneous surgery. J Am Acad Dermatol 2004;51:955-7.

8. Katz J, Feldman MA, Bass EB, et al. Risks and benefits of anticoagulant and antiplatelet medication use before cataract surgery. Ophthalmology 2003;110:1784-8.

9. Hammerstingl C, Schmitz A, Fimmers R, Omran H. Bridging of chronic oral anticoagulation with enoxaparin in patients with atrial fibrillation: results from the prospective BRAVE registry. Cardiovasc Ther 2009;27:230-8.

10. Robinson M, Healey JS, Eikelboom J, et al. Postoperative low-molecular-weight heparin bridging is associated with an increase in wound hematoma following surgery for pacemakers and implantable defibrillators. Pacing Clin Electrophysiol 2009;32:378-82.

11. Daniels PR, McBane RD, Litin SC, et al. Peri-procedural anticoagulation management of mechanical prosthetic heart valve patients. Thromb Res 2009;124:300-5.

12. Tolosana JM, Berne P, Mont L, et al. Preparation for pacemaker or implantable cardiac defibrillator implants in patients with high risk of thrombo-embolic events: oral anticoagulation or bridging with intravenous heparin? A prospective randomized trial. Eur Heart J 2009;30:1880-4.

13. Tischenko A, Gula L, Yee R, Klein GJ, Skanes AC, Krahn AD. Implantation of cardiac rhythm devices without interruption of oral anticoagulation compared with perioperative bridging with lowmolecular weight heparin. Am Heart J 2009;158:252-6.

14. Rhodes DA, Severson EP, Hodrick JT, Dunn HK, Hofmann AA. Discontinuation of warfarin is unnecessary in total knee arthroplasty. Clin Orthop Relat Res 2010;468:120-6.

15. Ercan M, Bostanci EB, Ozer I, et al. Postoperative hemorrhagic complications after elective laparoscopic cholecystectomy in patients receiving long-term anticoagulant therapy. Langenbecks Arch Surg 2010;395:247-53.

16. Chow V, Ranasinghe I, Lau J, et al. Peri-procedural anticoagulation and the incidence of haematoma formation after permanent pacemaker implantation in the elderly. Heart Lung Circ 2010;19:706-12.

17. Klamroth R, Gottstein S, Essers E, Landgraf H. Bridging with enoxaparin using a half-therapeutic dose regimen: safety and efficacy. Vasa 2010;39:243-8.

18. Dotan ZA, Mor Y, Leibovitch I, et al. The efficacy and safety of perioperative low molecular weight heparin substitution in patients on chronic oral anticoagulant therapy undergoing transurethral prostatectomy for bladder outlet obstruction. J Urol 2002;168:610-3.

19. Pengo V, Cucchini U, Denas G, et al. Standardized low-molecular-weight heparin bridging regimen in outpatients on oral anticoagulants undergoing invasive procedure or surgery: an inception cohort management study. Circulation 2009;119:2920-7. 
20. Dunn AS, Spyropoulos AC, Turpie AG. Bridging therapy in patients on long-term oral anticoagulants who require surgery: the Prospective Peri-operative Enoxaparin Cohort Trial (PROSPECT). J Thromb Haemost 2007;5:2211-8.

21. Omran H, Hammerstingl C, Paar WD. Perioperative Bridging with Enoxaparin. Results of the Prospective BRAVE Registry with 779 Patients. Med Klin (Munich) 2007;102:809-15.

22. Elzayat $E$, Habib E, Elhilali M. Holmium laser enucleation of the prostate in patients on anticoagulant therapy or with bleeding disorders. J Urol 2006;175:1428-32.

23. Tompkins C, Cheng A, Dalal D, et al. Dual antiplatelet therapy and heparin "bridging" significantly increase the risk of bleeding complications after pacemaker or implantable cardioverter-defibrillator device implantation. J Am Coll Cardiol 2010;55:2376-82.

24. Li HK, Chen FC, Rea RF, et al. No increased bleeding events with continuation of oral anticoagulation therapy for patients undergoing cardiac device procedure. Pacing Clin Electrophysiol 2011;34:868-74.

25. Ghanbari H, Feldman D, Schmidt $M$, et al. Cardiac resynchronization therapy device implantation in patients with therapeutic international normalized ratios. Pacing Clin Electrophysiol 2010;33:400-6.

26. Page SP, Siddiqui MS, Finlay $M$, et al. Catheter ablation for atrial fibrillation on uninterrupted warfarin: can it be done without echo guidance? J Cardiovasc Electrophysiol 2011;22:265-70.

27. Stang A. Critical evaluation of the Newcastle-Ottawa scale for the assessment of the quality of nonrandomized studies in meta-analyses. Eur J Epidemiol 2010;25:603-5.

28. Higgins J, Ed. Cochrane Handbook for Systematic Reviews of Interventions Version 5.1.0 [updated March 2011]. THe Cochrane Collaboration, 2011 [cited 2012 September 28]. Available from www.cochrane-handbook.org2011.

29. Higgins JP, Thompson SG, Deeks JJ, Altman DG. Measuring inconsistency in meta-analyses. BMJ 2003; 327:557-60.

30. Garcia DA, Regan S, Henault LE, et al. Risk of thromboembolism with short-term interruption of warfarin therapy. Arch Intern Med 2008;168:63-9.

31. Wazni OM, Beheiry S, Fahmy $\mathrm{T}$, et al. Atrial fibrillation ablation in patients with therapeutic international normalized ratio: comparison of strategies of anticoagulation management in the periprocedural period. Circulation. 2007;116:2531-4.

32. Cano O, Osca J, Sancho-Tello MJ, Olague J, Castro JE, Salvador A. Morbidity associated with three different antiplatelet regimens in patients undergoing implantation of cardiac rhythm management devices. Europace. 2011;13:395-401.

33. Krane LS, Laungani R, Satyanarayana R, et al. Robotic-assisted radical prostatectomy in patients receiving chronic anticoagulation therapy: role of perioperative bridging. Urology 2008;72:1351-5.

34. Ahmed I, Gertner E, Nelson WB, et al. Continuing warfarin therapy is superior to interrupting warfarin with or without bridging anticoagulation therapy in patients undergoing pacemaker and defibrillator implantation. Heart Rhythm 2010;7:745-9.

35. Manoukian SV. Predictors and impact of bleeding complications in percutaneous coronary intervention, acute coronary syndromes, and ST-segment elevation myocardial infarction. Am J Cardiol 2009;104:9-15.

36. Promberger R, Ott J, Kober F, et al. Risk factors for postoperative bleeding after thyroid surgery. Br J Surg 2012;99:373-9.

37. Birnie D, Healey JS, Krahn A, et al. Bridge or continue Coumadin for device surgery: a randomized controlled trial rationale and design. Curr Opin Cardiol 2009;24:82-7.

38. Ramirez A, Wall TS, Schmidt M, Selzman K, Daccarett M. Implantation of cardiac rhythm devices during concomitant anticoagulation or antiplatelet therapy. Expert Rev Cardiovasc Ther 2011;9: 609-14.

39. Jamula E, Douketis JD, Schulman S. Perioperative anticoagulation in patients having implantation of a cardiac pacemaker or defibrillator: a systematic review and practical management guide. J Thromb Haemost 2008;6:1615-21.

40. Levi $M$, Eerenberg $E$, Kamphuisen PW. Periprocedural reversal and bridging of anticoagulant treatment. Neth J Med 2011;69:268-73. 
41. Spyropoulos AC. To bridge or not to bridge: that is the question. The argument FOR bridging therapy in patients on oral anticoagulants requiring temporary interruption for elective procedures. J Thromb Thrombolysis 2010;29:192-8.

42. Gerotziafas GT, Dupont C, Spyropoulos AC, et al. Differential inhibition of thrombin generation by vitamin $\mathrm{K}$ antagonists alone and associated with low-molecular-weight heparin. Thromb Haemost 2009;102:42-8.

43. Douketis JD, Woods K, Foster GA, Crowther MA. Bridging anticoagulation with low-molecular-weight heparin after interruption of warfarin therapy is associated with a residual anticoagulant effect prior to surgery. Thromb Haemost 2005;94:528-31.

44. Jamula E, Lloyd NS, Schwalm JD, Airaksinen KE, Douketis JD. Safety of uninterrupted anticoagulation in patients requiring elective coronary angiography with or without percutaneous coronary intervention: a systematic review and metaanalysis. Chest 2010;138:840-7. 


\section{Appendix 3.1 The search strategy for MEDLINE and EMBASE}

(bridging OR perioperative prophylaxis OR periprocedural anticoagulant management OR periprocedural anticoagulant treatment) AND (anticoagulants OR antithrombotic agents OR vitamin k OR vitamin k antagonist OR 4-Hydroxycoumarins OR warfarin OR coumadin OR coumatetralyl OR phenprocoumon OR dicoumarol OR tioclomarol OR brodifacoum OR phenindione OR clorindione OR diphenadione OR Imwh OR low molecular weight heparin OR ardeparin OR certoparin OR enoxaparin OR parnaparin OR tinzaparin OR dalteparin OR reviparin OR nadroparin OR fondaparinux OR UFH OR unfractionated heparin OR heparin) AND (bleeding risk OR bleeding event OR bleeding complication OR thrombotic event OR TE OR stroke OR cerebrovascular incident OR CVA OR venous thromboembolism OR VTE OR pulmonary embolism OR myocardial infarction OR thrombo (wildcard)

Limits Activated MEDLINE: English, German, Dutch, MEDLINE, published in the last 10 years

Limits Activated EMBASE: English, German, Dutch, published in the last 10 years 


\section{Chapter 4}

\section{Practice of bridging anticoagulation; guideline adherence and risk factors for bleeding}

Pieter Eijgenraam,Hugo ten Cate, Arina J ten Cate-Hoek

Neth J Med. 2014;72:157-164 


\begin{abstract}

\section{Background}

Perioperative bridging with low molecular weight heparins (LMWH) is applied to minimize the risk of thromboembolism (TE). Guidelines characterize patients at risk and strategies to be followed. We assessed guideline adherence of bridging episodes and identified possible risk factors for bleeding in a retrospective cohort study.
\end{abstract}

\title{
Methods
}

We searched the electronic patient data system of the Maastricht anticoagulation service, the Netherlands. We identified 181 patients on chronic anticoagulation who underwent surgery (222 procedures) and were bridged with LMWH. Guideline adherence was defined in terms of the relation between TE risk and the dose of LMWH administered, the bleeding risk of the procedure and the duration of postprocedural administration of LMWH. Logistic regression was used to identify risk factors for bleeding.

\section{Results}

Of all low TE risk patients ( $n=102$ ) $84.3 \%$ was treated with therapeutic doses of LMWH. The median duration of postprocedural LMWH administration was eight days. The 30-day incidence of major bleeding in the entire group $(n=222)$ was $11.3 \%$. Two patients $(0.90 \%)$ experienced a deep venous thrombosis. Creatinine clearance $\leq 40 \mathrm{ml} / \mathrm{min}$ (odds ratio (OR) $5.03,95 \%$ confidence interval (CI) 1.25 to 20.26 ) and dental procedures (OR $3.32,95 \% \mathrm{Cl} 1.22$ to 9.04 ) were independent predictors for total bleeding.

\section{Conclusion}

Guideline adherence was low, leading to prolonged bridging procedures, excess treatment of patients and high bleeding rates. The majority of patients had a low thromboembolic risk profile or underwent low risk procedures. For patients with decreased creatinine clearance reduced doses of LMWH should be considered to reduce bleeding risk. 


\section{Introduction}

Perioperative interruption of chronic anticoagulation harbours the risk of thromboembolism (TE). To minimize the risk for TE during the anticoagulant free interval, bridging therapy with low molecular weight heparins (LMWH) is applied. This introduces the risk of bleeding. Vitamin $\mathrm{K}$ antagonists (VKA) are administered to patients at increased risk of a venous or arterial TE due to for instance venous thromboembolism, atrial fibrillation, mechanical heart valves, or stroke. According to current guidelines LMWH or unfractionated heparin (UFH) in therapeutic dosages is the preferred anticoagulant for high TE risk patients undergoing high bleeding risk surgery. For patients with an intermediate TE risk profile two options are available: therapeutic or prophylactic dosages of LMWH. For patients at low TE risk again two options are available: prophylactic dosages or interruption of VKA without LMWH or UFH. In low bleeding risk procedures VKA should not be interrupted. The perioperative administration of UFH or LMWH as a part of bridging therapy possibly leads to increased bleeding risk associated with sometimes severe consequences like intracranial bleeding with major disability or even death as a result. ${ }^{1-4}$ Although guidelines characterize patients at risk and advise on strategies to be followed, the application of bridging therapy is often guideline discordant. Literature reveals that a wide range of approaches to bridging anticoagulation is in use, possibly due to unfamiliarity with the current guidelines and the weak evidence on which they are based. ${ }^{5,6}$ Evidence from randomized trials is lacking, probably because ethical issues might arise during the design of such a study; available evidence is therefore mainly based on observational studies. The incidence of postoperative total bleeding ranges from $4.1 \%$ to $25 \%$ in populations subject to diverse bridging regimes undergoing different surgical interventions. ${ }^{4,7-9}$ The incidence of TE ranges from $0 \%$ to $2.6 \%$ in different studies. ${ }^{3,4,10-13}$ Apart from improvement of adherence to guidelines there is a need of defining patient and procedure related characteristics which might fine-tune the bridging strategy and thereby decrease the incidence of periprocedural bleeding and TE. Until now, renal insufficiency, ${ }^{1,14} \mathrm{CHADS}_{2}$ (a composite score of congestive heart failure, hypertension, age, diabetes and stroke), ${ }^{8,9,13}$ mitral valve replacement, ${ }^{4,15,16}$ thrombocytopenia, ${ }^{8,16}$ LMWH administration within 24 hours after the procedure, ${ }^{16,17}$ increasing age, ${ }^{7,13}$ and total duration of periprocedural heparin use $^{18}$ are the only consistent risk factors for bleeding. Baseline INR in bridging groups is not associated with postoperative bleeding in different studies. ${ }^{7,19}$ It is still unclear if the application of bridging therapy results in decreased TE risk when compared to VKA cessation alone or VKA continuation without the administration of $\mathrm{LMWH} .{ }^{20}$ 
Our primary goal was to delineate the features of bridging strategies applied in the region around Maastricht, the Netherlands. We intended to assess guideline adherence and to document the incidence of bridging-related bleeding and TE. The secondary goal was to identify possible risk factors for bleeding during bridging therapy, both patient related risk factors and risk factors associated with the bridging strategy itself.

\section{Methods}

\section{Cohort}

To determine guideline adherence and identify risk factors for major and total bleeding, we retrospectively searched the electronic patient data system of the Maastricht anticoagulation service for the interval of September 2010 until June 2012. This database contains data of approximately 4200 patients in relation to VKA therapy and bleeding complications. Additional medical information for these patients was retrieved from the patient database of the Maastricht University Medical Centre (MUMC+). Institutional review board approval was obtained (METC 11-4-140).

We identified 181 patients (222 procedures) on chronic anticoagulation who received bridging therapy. A broad definition of bridging therapy was used: any period of periprocedural cessation of VKA including the day of the intervention and administration of any dose of periprocedural LMWH or UFH. The following inclusion criteria were determined: 1) participants were bridged as defined above and 2) were on chronic VKA treatment, initiated more than three months ago. Participants, who had 1) additional surgery within 30 days, 2) underwent an emergency procedure, or 3) yielded inconsistent data, (i.e. contradictory information was found in the different databases), were excluded. We sought to report our study according to the recommendations for reporting studies in periprocedural antithrombotic and bridging therapy issued by the ISTH. ${ }^{21}$

\section{Guideline adherence}

We determined guideline adherence of bridging episodes; we ascertained the proportion of patients bridged according to the ACCP guidelines for perioperative management of antithrombotic therapy $2008 .^{22}$ These guidelines are officially adopted and propagated in the MUMC+. For surgical bleeding risk classification we made use of a two tier distribution in our descriptive analyses; Dental procedures, (extraction of 1-3 teeth, implant placement, surgical extraction of wisdom teeth, 
surgical root canal treatment, incision of an abscess, and dental hygiene treatment), cataract surgery, small dermatological interventions, and all other procedures with local anticoagulant options were considered low bleeding risk procedures making it possible to continue VKA treatment. All other procedures were qualified as high bleeding risk procedures and therefore warranted bridging anticoagulation according to ACCP guidelines, providing that elevated TE risk was established in the patient. Arterial and venous TE risks were defined as low, intermediate, or high. TE risk in general was defined as a composite score of venous and arterial TE risk. A low, intermediate, or high arterial/venous TE risk was defined as a low, intermediate, or high composite risk respectively; in case of exposure to both an arterial and venous risk the highest score on either risk was expressed as the TE risk. The variable TE risk in general was composed to be able to estimate the combined effect of arterial and venous TE risk on the physician's decision to administer therapeutic doses of LMWH and on bleeding risk in our univariable and multivariable analyses.

Guideline adherence was defined as low TE risk patients receiving prophylactic doses LMWH post procedurally and intermediate to high TE risk patients receiving prophylactic or therapeutic doses post procedurally. Patients without prior surgical bleedings undergoing low risk dental, cataract, or dermatological procedures should not be bridged; continuation of VKA is the preferred option, but for patients who have experienced a prior surgical bleeding bridging is indicated for these low risk procedures. The period of postoperative administration of LMWH should not exceed seven days. We determined the proportion of all patients on long-term acenocoumarol or phenprocoumon therapy, treated within our institution that received postprocedural therapeutic doses of $\mathrm{LMWH}$. In the literature this proportion ranges from $22 \%$ to $85 \%$ and is associated with major bleeding. ${ }^{6}$

\section{Complications}

We documented the incidence of perioperative total bleeding, major bleeding and TE from three days prior until 30 days after the procedure. Major bleeding was defined according to the criteria used by the Federatie van Nederlandse Trombosediensten (FNT) as any bleeding resulting in death, any intracranial bleeding, and any bleeding that leads to transfusion of packed red cells and/or treatment in a hospital or joint bleeds; all other bleedings including hematomas are qualified as minor bleedings. Thromboembolic complications are defined as any objectively confirmed TE, and death caused by TE; myocardial infarction and acute coronary syndrome were excluded due to the difficulty of attributing these events to cardio embolism in the perioperative setting. ${ }^{21}$ 


\section{Risk factors for bleeding}

Primary outcomes were total and major bleeding. Due to the low number of cases we were unable to identify patient characteristics associated with the risk of TE. To assess bleeding risk of the procedure we used in addition to the ACCP risk classification (low, high) the 5 point scale as proposed by Jaffer et al. ranging from minimal bleeding risk (score 1 ) to critical risk (score 5). ${ }^{6}$ This 5 point scale was used in our univariable and multivariable analyses as an independent variable. Creatinine clearance was divided into three categories: $>60,41-60$, and $\leq 40 \mathrm{ml} / \mathrm{min}$ for reasons of an approximate equal distribution of the obtained values among the categories. The postprocedural restart time of LMWH was estimated and rounded to 0.5 days (12 hours). The total duration of LMWH administration was calculated taking into account the intermediate period that the patient did not receive LMWH including the day of the intervention.

\section{Statistical analysis}

Descriptive statistics were used to determine patient and procedure characteristics. Continuous variables are reported as means, their standard deviations (SD), and median values; categorical data are presented as counts and percentages. To assess whether the bleeding risk of the intervention (score 1-5), TE risk (low, intermediate, or high), creatinine clearance ( $\mathrm{ml} / \mathrm{min})$, or age (years) influenced the physician's decision to administer therapeutic dosages of LMWH post procedurally, univariable and multivariable logistic regression were performed with therapeutic dosage of postprocedural LMWH as the outcome.

In order to identify risk factors for total and major bleeding, first univariable and subsequently multivariable logistic regression was applied. For our multivariable models with both total bleeding and major bleeding as outcomes we selected the established risk factors age (years), ${ }^{7,13}$ total duration of periprocedural heparin use (days), ${ }^{18}$ and the variables associated in univariable analysis $(p<0.10)$ with total bleeding: dental procedures (yes/no), TE risk (low, intermediate or high), and creatinine clearance $(\mathrm{ml} / \mathrm{min})$. In the univariable and multivariable analyses missing values were imputed; we opted for multiple imputations. Besides the original dataset five additional datasets were created using the Chain Monte Carlo Markov method. The results of these six datasets were pooled. To assess the fitting of different models Hosmer-Lemeshov and model chi-square statistic tests were performed. Risks are expressed as odds ratios (OR) and $p$-values for linear trends are presented. A two sided $p$-value $<0.05$ was considered statistically significant. Data were analyzed with SPSS version 19.0 .0 


\section{Results}

We were unable to classify 12 participants (12 procedures) in any TE risk category; According to the ACCP guidelines these patients were not indicated for VKA use; conditions like thrombophilia without previous venous thromboembolism (VTE) and cardiomyopathy are not mentioned in the risk scheme.

\section{Baseline characteristics}

Baseline clinical characteristics are detailed in Table 4.1. The average age was 70.3 years (standard deviation (SD) 11.4) and 59.0\% were male. Arterial TE risk was the indication for VKA use in 190 patients (85.6\%); low risk atrial fibrillation (AF) with $\mathrm{CHADS}_{2}$ scores 0-1 was the most prevalent condition in 67/190 patients (35.3\%). VTE risk was present in 42 patients (18.9\%); the most prevalent condition was VTE more than six months ago: $32 / 42$ (76.2\%). 10 patients had both an arterial and venous indication for VKA therapy. Creatinine clearance was decreased ( $\leq 60 \mathrm{ml} / \mathrm{min}$ ) in $62 / 222(27.9 \%)$ of the patients and in $62 / 126$ (49.2\%) of the measurements perfor+med. In 96 (43.2\%) patients no periprocedural creatinine clearance was determined.

Table 4.1 Baseline and procedure characteristics, anticoagulation and complications

\begin{tabular}{|c|c|c|}
\hline \multicolumn{3}{|l|}{ Baseline characteristics } \\
\hline Men & & $131(59.0 \%)$ \\
\hline Age (years) & & $70.3 \pm 11.4$ \\
\hline \multirow[t]{4}{*}{ Arterial TE risk $(n=190)$} & High & $42(27.4 \%)$ \\
\hline & Intermediate & $56(29.5 \%)$ \\
\hline & Low & $80(42.1 \%)$ \\
\hline & Not mentioned in $\mathrm{ACCP} / \mathrm{CBO}$ guidelines & $12(6.3 \%)$ \\
\hline \multirow[t]{3}{*}{ Venous TE risk $(n=42)$} & High & $9(21.4 \%)$ \\
\hline & Intermediate & $1(2.4 \%)$ \\
\hline & Low & $32(76.2 \%)$ \\
\hline \multirow[t]{4}{*}{ Creatinine clearance $(n=222)$} & $>60 \mathrm{ml} / \mathrm{min}$ & $64(28.8 \%)$ \\
\hline & $41-60 \mathrm{ml} / \mathrm{min}$ & $42(18.9 \%)$ \\
\hline & $\leq 40 \mathrm{ml} / \mathrm{min}$ & $20(9.0 \%)$ \\
\hline & No measurement performed & $96(43.2 \%)$ \\
\hline \multicolumn{3}{|c|}{ Procedure characteristics } \\
\hline \multirow[t]{5}{*}{ Bleeding risk procedures ACCP $(n=222)$} & High & $160(72.1 \%)$ \\
\hline & High bleeding risk procedure & $157(70.7 \%)$ \\
\hline & Bleeding previous surgery & $3(1.4 \%)$ \\
\hline & low & $62(27.9 \%)$ \\
\hline & Low bleeding risk procedure & $62(27.9 \%)$ \\
\hline \multirow[t]{5}{*}{ Bleeding risk 5 point scale $(n=222)$} & Score 1 & $143(64.4 \%)$ \\
\hline & Score 2 & 33 (14.9\%) \\
\hline & Score 3 & 39 (17.6\%) \\
\hline & Score 4 & $7(3.2 \%)$ \\
\hline & Score 5 & $0(0.0 \%)$ \\
\hline
\end{tabular}


Table 4.1 (continued)

\begin{tabular}{|c|c|c|}
\hline \multicolumn{3}{|l|}{ Anticoagulation characteristics } \\
\hline \multirow[t]{2}{*}{ VKA $(n=222)$} & Acenocoumarol & $200(90.1 \%)$ \\
\hline & Phenprocoumon & $22(9.9 \%)$ \\
\hline \multirow[t]{2}{*}{ Vitamin K preprocedural $(n=6)$} & Acenocoumarol & $0(0.0 \%)$ \\
\hline & Phenprocoumon & $6(100 \%)$ \\
\hline \multirow[t]{2}{*}{ LMWH postprocedural $(n=222)$} & Prophylactic & $23(10.4 \%)$ \\
\hline & Therapeutic & $199(89.6 \%)$ \\
\hline \multirow[t]{2}{*}{ Stop time VKA (days) } & Acenocoumarol & $-3.4 \pm 1.6$ Median: -3.0 \\
\hline & Phenprocoumon & $-5.3 \pm 3.6$ Median: -5.0 \\
\hline \multirow{2}{*}{$\begin{array}{l}\text { Restart time VKA postprocedural } \\
\text { (days) }\end{array}$} & Acenocoumarol & $1.4 \pm 3.3$ Median: 0.0 \\
\hline & Phenprocoumon & $2.1 \pm 8.0$ Median: 0.0 \\
\hline \multirow[t]{2}{*}{ Start time LMWH preprocedural (days) } & Acenocoumarol & $-3.2 \pm 1.7$ Median: -3.0 \\
\hline & Phenprocoumon & $-6.3 \pm 5.1$ Median: -4.0 \\
\hline \multirow[t]{2}{*}{ Stop time LMWH preprocedural (days) } & Acenocoumarol & $-0.9 \pm 0.5$ Median: -1.0 \\
\hline & Phenprocoumon & $-1.3 \pm 0.6$ Median: -1.0 \\
\hline \multirow{2}{*}{$\begin{array}{l}\text { Restart time LMWH postprocedural } \\
\text { (hours) }\end{array}$} & Acenocoumarol & $19.3 \pm 9.9$ Median: 24.0 \\
\hline & Phenprocoumon & $19.3 \pm 9.5$ Median: 12.0 \\
\hline \multirow{2}{*}{$\begin{array}{l}\text { Stop time LMWH postprocedural } \\
\text { (days) }\end{array}$} & Acenocoumarol & $9.6 \pm 6.0$ Median: 8.0 \\
\hline & Phenprocoumon & $13.9 \pm 11.9$ Median: 10.0 \\
\hline \multirow[t]{2}{*}{ Total duration LMWH (days) } & Acenocoumarol & $11.2 \pm 6.2$ Median: 8.5 \\
\hline & Phenprocoumon & $17.6 \pm 13.8$ Median: 13.0 \\
\hline \multirow[t]{2}{*}{ INR day intervention } & Acenocoumarol & $1.1 \pm 0.1$ \\
\hline & Phenprocoumon & $1.2 \pm 0.2$ \\
\hline \multirow[t]{2}{*}{ Time INR>2 (days) } & Acenocoumarol & $8.7 \pm 6.9$ Median: 7.0 \\
\hline & Phenprocoumon & $11.8 \pm 10.5$ Median: 8.0 \\
\hline \multicolumn{3}{|c|}{ Low TE risk and postprocedural LMWH dosage } \\
\hline \multicolumn{2}{|c|}{$\begin{array}{l}\text { Low TE risk }(n=102) \text { and prophylactic } \\
\text { dose }\end{array}$} & $16(15.7 \%)$ \\
\hline \multicolumn{2}{|l|}{$\begin{array}{l}\text { Low TE risk }(n=102) \text { and therapeutic } \\
\text { dose }\end{array}$} & $86(84.3 \%)$ \\
\hline \multicolumn{3}{|l|}{ Complications } \\
\hline \multirow[t]{3}{*}{ Bleeding $(n=44)$} & Transfusion & $4(1.8 \%)$ \\
\hline & Hospital treatment & $21(9.5 \%)$ \\
\hline & Minor & $19(8.6 \%)$ \\
\hline
\end{tabular}

Abbreviations: ACCP, American College of Chest Physicians; AF, atrial fibrillation; CBO, Centraal BegeleidingsOrgaan voor de intercollegiale toetsing; $\mathrm{CHADS}_{2}$, congestive heart failure, hypertension, age, diabetes and stroke(2); INR, International Normalized Ratio; LMWH, low molecular weight heparin; MHV, mechanical heart valve; TE, thromboembolism; VKA vitamin $\mathrm{K}$ antagonist; VTE, venous thromboembolism

\section{Procedure characteristics}

Procedure characteristics are detailed in table 1; 222 procedures were performed in 181 patients. In 62 (27.9\%) of all cases bridging therapy was applied for a procedure for which bridging was not indicated; all were low risk dental, cataract, or dermatological interventions. A variety of inpatient and outpatient procedures was performed: dental procedures, gastroscopies, and colonoscopies were the most prevalent interventions, see Table 4.2 . The majority $(143 ; 64.4 \%)$ of all procedures 
was classified as minimal bleeding risk procedure according to the Jaffer scale (score 1), no procedures were classified as critical risk (score 5), only seven (3.2\%) procedures were assessed as major bleeding risk (score 4). 17 participants underwent two procedures, seven participants underwent three, and two participants underwent four procedures.

Table 4.2 Procedures performed $(n=222)$

\begin{tabular}{|c|c|}
\hline Gastrointestinal & \\
\hline Endoscopy colon/duodenum with or without biopsy & 26 \\
\hline Cholecystectomy & 2 \\
\hline Abdominal surgery & 7 \\
\hline Haemorrhoids & 3 \\
\hline Colon polyp removal & 1 \\
\hline Orthopaedic & \\
\hline Total hip arthroplasty & 5 \\
\hline Total knee arthroplasty & 4 \\
\hline Intra-articular injections & 3 \\
\hline Elbow/foot/shoulder surgery & 3 \\
\hline Other & 8 \\
\hline Urology & \\
\hline Prostate biopsy & 7 \\
\hline TUR-prostate & 4 \\
\hline Bladder cancer surgery & 3 \\
\hline Brachytherapy & 2 \\
\hline Kidney scope procedure & 5 \\
\hline Cystoscopy with or without biopsy & 2 \\
\hline Other & 7 \\
\hline Dental & \\
\hline Extractions & 37 \\
\hline Implants & 10 \\
\hline Dental hygiene treatment & 1 \\
\hline Neurosurgical & \\
\hline Hernia nuclei pulposi surgery & 5 \\
\hline Lumbar puncture & 1 \\
\hline Vascular & \\
\hline Varices & 2 \\
\hline Angioplasty/stent placement & 2 \\
\hline Bypass surgery & 1 \\
\hline Plastic & \\
\hline Hand surgery & 6 \\
\hline Dermatologic procedure & 8 \\
\hline Entropion surgery & 3 \\
\hline Other & 3 \\
\hline Interventional radiology & \\
\hline Heart biopsy & 2 \\
\hline Cardiac catherization & 9 \\
\hline Other & 2 \\
\hline
\end{tabular}


Chapter 4

\begin{tabular}{lr} 
Table $4.2 \quad$ (continued) \\
\hline Other \\
ENT surgery \\
Neurolysis & 2 \\
Cataract & 2 \\
Hernia umbilicalis/inguinalis & 4 \\
Breast cancer & 4 \\
Breast biopsy & 6 \\
Bronchoscopy with or without biopsy & 4 \\
Other & 5 \\
\hline
\end{tabular}

Abbreviations: ENT, ear, nose and throat; TUR, transurethral resection of the prostate

\section{Anticoagulation}

Anticoagulation characteristics are detailed in Table 4.1. The majority of the patients used acenocoumarol as oral anticoagulant: 200 (90.1\%), the remaining 22 (9.9\%) used phenprocoumon. The median preoperative stop time of VKA was day -3 (mean -3.4, SD 1.6) and day -5 (mean -5.3, SD 3.6) for acenocoumarol and phenprocoumon, respectively. Vitamin $\mathrm{K}$ was used to reverse anticoagulation only in six patients $(2.7 \%)$; all used phenprocoumon, a VKA with a relative long half-life of 120-200 hours. Acenocoumarol was resumed a median of 0 days (mean 1.4, SD 1.6); phenprocoumon was resumed a median of 0 days (mean 2.1, SD 8.0). LMWH was used as bridging agent of first choice in all patients. The proportion of patients at low TE risk $(n=102)$ treated with therapeutic doses of LMWH post procedurally was $84.3 \%(n=86)$. We also explored periprocedural timing of LMWH administration; LMWH therapy was initiated a median 3 days (mean 3.2, SD 1.7) and a median 4 days (mean 6.3, SD 5.1) before and stopped a median of 1 (mean 0.9, SD 0.5 and mean 1.3, SD 0.6) day prior to the planned procedure in acenocoumarol and phenprocoumon users respectively. The median time of postoperative restart of LMWH therapy was 24 hours (mean 19.3, SD 9.9) and 12 hours (mean 19.3, SD 9.5) in acenocoumarol and phenprocoumon users respectively. The median duration of postoperative $\mathrm{LMWH}$ administration was 8 days (mean 11.2, SD 6.2) and 13 days (mean: 17.6, SD 13.8) in acenocoumarol and phenprocoumon users respectively. Of all patients undergoing bridging therapy 199 (89.6\%) were treated with therapeutic dosages of LMWH after the procedure.

Univariable logistic regression with postprocedural therapeutic dosage of LMWH as the outcome resulted in non significant effects for all variables. No proof was found that the prescribing physician's decision to administer therapeutic dosages of LMWHs was influenced by age, TE risk, surgical bleeding risk, or creatinine clearance. Patients at high TE risk compared to low risk patients had a non significant higher risk of exposure to therapeutic doses (OR $4.22,95 \% \mathrm{Cl} 0.93$ to 19.24 ), $\mathrm{p}$ for linear trend=0.06. 
Patients with a creatinine clearance within the range of 41-60 $\mathrm{ml} / \mathrm{min}$ compared to a clearance $>60 \mathrm{ml} / \mathrm{min}$ had a non significant lower risk of exposure to therapeutic doses (OR $0.43,95 \% \mathrm{Cl} 0.10$ to 1.82 ), $\mathrm{p}$ for linear trend=0.88. A high bleeding risk procedure (score 4 ) on the Jaffer scale compared to a procedure score of 1 resulted in a non significant decreased risk of exposure to therapeutic doses of LMWH (OR 0.24, $95 \% \mathrm{Cl} 0.04$ to 1.36 ), $\mathrm{p}$ for linear trend=0.11. Multivariable analyses including the aforementioned variables resulted in overall non significant results. Patients at high TE risk compared to low risk had a borderline non significant higher risk of exposure to therapeutic dosages after their intervention (OR 4.96, 95\% $\mathrm{Cl} 0.97$ to 25.26), p for linear trend $=0.05$. Patients with a creatinine clearance within the range of $41-60$ $\mathrm{ml} / \mathrm{min}$ compared to clearance $>60 \mathrm{ml} / \mathrm{min}$ had a non significant lower risk of exposure to therapeutic doses (OR $0.38,95 \% \mathrm{Cl} 0.08$ to 1.81 ), p for trend $=0.73$. Finally, a bleeding risk score 4 compared to score 1 resulted in a non significant decreased risk (OR $0.27,95 \% \mathrm{Cl} 0.04$ to 1.86 ), $\mathrm{p}$ for linear trend=0.23. The goodness of fit of the model was assessed, resulting in a p-value of 0.77 on the Hosmer-Lemeshov test and the model chi-square statistic resulted in a p-value of 0.75 .

\section{Complications}

The 30-day incidence of total bleeding in the entire group of procedures performed was $44(19.8 \%)$, the incidence of major bleeding 25 (11.3\%); there were no deaths, no intracranial bleedings, four patients required a transfusion, and 21 had to be treated in a hospital due to postoperative bleeding. Two patients $(0.9 \%)$ experienced a deep venous thrombosis and recovered; see Table 4.1.

\section{Risk factors for bleeding}

Univariable logistic regression analysis revealed high versus low TE risk (OR 2.59, 95\% $\mathrm{Cl} 1.11$ to 6.04), $\mathrm{p}$ for linear trend=0.03 and dental procedures (OR 2.98, 95\% Cl 1.45 to 6.13) as risk factors for total bleeding; all other results are non significant. Creatinine clearance $\leq 40$ versus $>60 \mathrm{ml} / \mathrm{min}$ and intermediate versus low TE risk resulted in non-significant elevated risks: OR 2.35, 95\% ( $\mathrm{Cl} 0.93$ to 5.90), p for linear trend $=0.07$ and $\mathrm{OR} 1.83,95 \% \mathrm{Cl} 0.77$ to 4.33 , respectively. To minimize the risk of reversed causality we excluded 18 cases in which VKA were stopped and LMWH administration due to a total bleeding was prolonged; the initial significantly increased risk caused by the total duration of LMWH administration (result not shown) disappeared: (OR $0.94,95 \% \mathrm{Cl} 0.86$ to 1.03). After exclusion of the aforementioned 18 cases, dental procedures (OR 3.32, 95\% Cl 1.22 to 9.04) and creatinine clearance $\leq 40$ versus $>60 \mathrm{ml} / \mathrm{min}(\mathrm{OR} 5.03,95 \% \mathrm{Cl} 1.25$ to 20.26), $\mathrm{p}$ for linear trend $=0.02$ were 
identified as independent predictors of total bleeding in a model completed with the variables age, duration of periprocedural use of $\mathrm{LMWH}$, and TE risk. The HosmerLemeshov test resulted in a $p$-value of 0.47 and the model chi-square statistic yielded a significant result: $p=0.01$.

Finally, we explored major bleeding. Univariable logistic regression revealed intermediate versus low TE risk as a risk factor (OR 3.40, 95\% Cl 1.17 to 9.93), p for linear trend $=0.11$. No further risk factors were identified. Our dataset contained only three high TE risk patients due to mitral valve replacements of which one experienced a major bleeding; hospital treatment was necessary (OR 4.06, 95\% Cl 0.36 to 46.50). In multivariable analysis, using the same model, we again excluded the aforementioned 18 cases to avoid differential misclassification and no significant risk factors were identified. The model as a whole scored a p-value of 0.83 on the Hosmer-Lemeshov test with a $p$-value of 0.50 on the model chi-square statistic.

\section{Discussion}

In our study we found that guideline adherence to bridging therapy in the region around Maastricht, the Netherlands, is not optimal. The most striking finding is that 84.3\% of all low TE risk patients was bridged with therapeutic doses LMWH. Low TE risk does not warrant bridging therapy and certainly not with therapeutic doses of LMWH. ${ }^{22}$ Furthermore we found compared to other studies, high rates of total and major bleedings. ${ }^{6,9,23,24}$ We were unable to find an association of this observed aggressive treatment with anticoagulants and the high bleeding rates, possibly due a lack of contrast in our population. Studies performed by Robinson et al. and Jaffer et al. identified postprocedural therapeutic doses of LMWH as a risk factor for bleeding. ${ }^{6,11}$ In general, bridging therapy exposes the patient to additional risks, also including a risk of heparin-induced thrombocytopenia (HIT). ${ }^{25}$ Interventions for which no bridging anticoagulation is indicated and VKA administration can simply be continued, represented $27.9 \%$ of the total number of procedures performed in our cohort; a fairly high proportion. Possibly due to the fact that the majority of the participants were outpatients, the period of exposure to LMWH was much longer than necessary according to the ACCP guidelines; in outpatients rigidly performed INR testing is often not feasible. ${ }^{26}$ Furthermore, due to a change in the anticoagulant regime in the outpatient setting the patient's compliance might be at risk; this might introduce an additional risk factor for bleeding or TE. Another possible explanation for prolonged LMWH administration might be the use of too low restart doses of VKA (i.e. the maintenance dose) instead of 1,5 to 2 times higher doses of acenocoumarol and 
phenprocoumon as advised in guidelines issued by the FNT. ${ }^{27}$ Overall TE incidence was low and in concordance with some other studies ${ }^{6,9,23,24}$; no arterial TE occurred. We conclude that individual clinicians often do not act according to the current bridging guidelines; in the observed cohort the decision to administer therapeutic dosages of LMWH was not or barely influenced by surgical bleeding risk, TE risk, or renal insufficiency. Krahn et al. and Skolarus et al. report similar findings ${ }^{5,28}$; Gerson et al. on the other hand concluded that most people receiving bridging therapy were managed according to current society guidelines. ${ }^{29}$ Possible explanations for non adherence are the lack of familiarity with these guidelines, lack of awareness of the significance of consistent bridging practices, disagreement with the guidelines, and resistance to change. ${ }^{30}$ It is also conceivable that physicians tend to over treat patients because the threat of a TE is considered more severe than the threat of bleeding. ${ }^{5}$

Renal insufficiency appeared an independent predictor for total bleeding. Other studies support this finding ${ }^{1,14}$; the clearance of $\mathrm{LMWH}$ is primarily renal, the plasma half-live increases in patients with renal failure and dose reduction is advised in these patients following the Cockroft Gault formula. ${ }^{8,31}$ As far as we know only one study performed by Hammerstingl reported high TE risk as a risk factor for perioperative bleeding. ${ }^{8}$ Possibly confounding biased this finding since in our analysis increasing TE risk was only found to be a risk factor for total bleeding in univariable analyses. An unexpected, novel finding is that dental treatment inflicts a very high bleeding risk on patients; most dental treatments do not warrant bridging therapy; instead, VKA continuation in combination with the oral administration of antifibrinolytic agents like tranexaminic acid are advised. ${ }^{22}$ Several studies report that restarting LMWH in close proximity to the intervention might induce bleeding. ${ }^{16,17}$ Our study does not support these findings; the observed high rate $(28.8 \%)$ of missing values concerning this variable might have diluted this effect.

\section{Strengths and limitations of study}

Our study has some weaknesses; the sample size was small and data were analyzed retrospectively. We were unable to compare different institutions with respect to guideline adherence, so only a local view on bridging practices could be provided. The strengths of our study are: a well defined study population and the observational design that allowed us to establish guideline adherence and identify risk factors for bleeding. We allow comparison of our results with other studies because we reported according to the recommendations for reporting studies in periprocedural antithrombotic and bridging therapy, issued by the ISTH. 


\section{Conclusions}

Guideline adherence to bridging therapy is poor in the observed single regional setting. This results in patients being unnecessarily exposed to LMWH and for too long periods of time. Since bridging is in general associated with increased bleeding risks, ${ }^{20,32}$ it should be avoided in the absence of a good indication. The additional omission of risk stratification based on assessment of renal function further increased bleeding rates. Although these observations are confined to a limited region within one country, there is no reason to expect that this represents a unique and regional problem. Rather, it illustrates the importance of adhering to guidelines for antithrombotic management. 


\section{References}

1. Cano O, Osca J, Sancho-Tello MJ, Olague J, Castro JE, Salvador A. Morbidity associated with three different antiplatelet regimens in patients undergoing implantation of cardiac rhythm management devices. Europace. 2011;13:395-401.

2. Tompkins C, Cheng A, Dalal D, et al. Dual antiplatelet therapy and heparin "bridging" significantly increase the risk of bleeding complications after pacemaker or implantable cardioverter-defibrillator device implantation. J Am Coll Cardiol. 2010;55:2376-82.

3. Li HK, Chen FC, Rea RF, et al. No increased bleeding events with continuation of oral anticoagulation therapy for patients undergoing cardiac device procedure. Pacing Clin Electrophysiol. 2011;34:868-74.

4. Ghanbari H, Feldman D, Schmidt $M$, et al. Cardiac resynchronization therapy device implantation in patients with therapeutic international normalized ratios. Pacing Clin Electrophysiol. 2010;33:400-6.

5. Krahn AD, Healey JS, Simpson CS, Essebag V, Sivakumaran S, Birnie DH. Anticoagulation of patients on chronic warfarin undergoing arrhythmia device surgery: wide variability of perioperative bridging in Canada. Heart Rhythm. 2009;6:1276-9.

6. Jaffer AK, Brotman DJ, Bash LD, Mahmood SK, Lott B, White RH. Variations in perioperative warfarin management: outcomes and practice patterns at nine hospitals. Am J Med. 2010;123:141-50.

7. Ercan M, Bostanci EB, Ozer I, et al. Postoperative hemorrhagic complications after elective laparoscopic cholecystectomy in patients receiving long-term anticoagulant therapy. Langenbecks Arch Surg. 2010;395:247-53.

8. Hammerstingl C, Omran H. Perioperative bridging of chronic oral anticoagulation in patients undergoing pacemaker implantation--a study in 200 patients. Europace. 2011;13:1304-10.

9. Hammerstingl C, Omran H. Bridging of oral anticoagulation with low-molecular-weight heparin: experience in 373 patients with renal insufficiency undergoing invasive procedures. Thromb Haemost. 2009;101:1085-90.

10. Hammerstingl C, Schmitz A, Fimmers R, Omran H. Bridging of chronic oral anticoagulation with enoxaparin in patients with atrial fibrillation: results from the prospective BRAVE registry. Cardiovasc Ther. 2009;27:230-8.

11. Robinson M, Healey JS, Eikelboom J, et al. Postoperative low-molecular-weight heparin bridging is associated with an increase in wound hematoma following surgery for pacemakers and implantable defibrillators. Pacing Clin Electrophysiol. 2009;32:378-82.

12. Tolosana JM, Berne $\mathrm{P}$, Mont $\mathrm{L}$, et al. Preparation for pacemaker or implantable cardiac defibrillator implants in patients with high risk of thrombo-embolic events: oral anticoagulation or bridging with intravenous heparin? A prospective randomized trial. Eur Heart J. 2009;30:1880-4.

13. Tischenko A, Gula LJ, Yee R, Klein GJ, Skanes AC, Krahn AD. Implantation of cardiac rhythm devices without interruption of oral anticoagulation compared with perioperative bridging with lowmolecular weight heparin. Am Heart J. 2009;158:252-6.

14. Ahmed I, Gertner E, Nelson WB, House CM, Zhu DW. Chronic kidney disease is an independent predictor of pocket hematoma after pacemaker and defibrillator implantation. J Interv Card Electrophysiol. 2010;29:203-7.

15. Steger V, Bail DH, Graf D, Walker T, Rittig K, Ziemer G. A practical approach for bridging anticoagulation after mechanical heart valve replacement. J Heart Valve Dis. 2008;17:335-42.

16. Tafur AJ, McBane R, 2nd, Wysokinski WE, et al. Predictors of major bleeding in peri-procedural anticoagulation management. J Thromb Haemost. 2012;10:261-7.

17. Chow V, Ranasinghe I, Lau J, et al. Peri-procedural anticoagulation and the incidence of haematoma formation after permanent pacemaker implantation in the elderly. Heart Lung Circ. 2010;19:706-12.

18. Kiviniemi $\mathrm{T}$, Karjalainen $\mathrm{P}$, Pietila $\mathrm{M}$, et al. Comparison of additional versus no additional heparin during therapeutic oral anticoagulation in patients undergoing percutaneous coronary intervention. Am J Cardiol. 2012;110:30-5.

19. Lahtela H, Rubboli A, Schlitt A, et al. Heparin bridging vs. uninterrupted oral anticoagulation in patients with Atrial Fibrillation undergoing Coronary Artery Stenting. Results from the AFCAS registry. Circ J. 2012;76:1363-8. 
20. Eijgenraam P, Ten Cate H, Ten Cate-Hoek AJ. Safety and Efficacy of Bridging with Low Molecular Weight Heparins: A Systematic Review and Partial Meta-Analysis. Curr Pharm Des. 2012.

21. Spyropoulos AC, Douketis JD, Gerotziafas G, Kaatz S, Ortel TL, Schulman S. Periprocedural antithrombotic and bridging therapy: recommendations for standardized reporting in patients with arterial indications for chronic oral anticoagulant therapy. J Thromb Haemost. 2012;10:692-4.

22. Douketis JD, Berger PB, Dunn AS, et al. The perioperative management of antithrombotic therapy: American College of Chest Physicians Evidence-Based Clinical Practice Guidelines (8th Edition). Chest. 2008;133:299-339.

23. Pengo V, Cucchini U, Denas G, et al. Standardized low-molecular-weight heparin bridging regimen in outpatients on oral anticoagulants undergoing invasive procedure or surgery: an inception cohort management study. Circulation. 2009;119:2920-7.

24. Malato A, Saccullo G, Lo Coco L, et al. Patients requiring interruption of long-term oral anticoagulant therapy: the use of fixed sub-therapeutic doses of low-molecular-weight heparin. J Thromb Haemost. 2010;8:107-13.

25. Jaffer AK, Brotman DJ, Chukwumerije N. When patients on warfarin need surgery. Cleve Clin J Med. 2003;70:973-84.

26. Deerhake JP, Merz JC, Cooper JV, Eagle KA, Fay WP. The duration of anticoagulation bridging therapy in clinical practice may significantly exceed that observed in clinical trials. J Thromb Thrombolysis. 2007;23:107-13.

27. Dolder BDv. De kunst van het doseren. Voorschoten: Federatie van Nederlandse trombosediensten; 2010.

28. Skolarus LE, Morgenstern LB, Froehlich JB, Lisabeth LD, Brown DL. Guideline-discordant periprocedural interruptions in warfarin therapy. Circ Cardiovasc Qual Outcomes. 2011;4:206-10.

29. Gerson LB, Michaels L, Ullah N, Gage B, Williams L. Adverse events associated with anticoagulation therapy in the periendoscopic period. Gastrointest Endosc. 2010;71:1211-7.

30. Eisenstein DH. Anticoagulation management in the ambulatory surgical setting. AORN J. 2012;95:51021 examination 22-4.

31. Harder S, Klinkhardt U, Alvarez JM. Avoidance of bleeding during surgery in patients receiving anticoagulant and/or antiplatelet therapy: pharmacokinetic and pharmacodynamic considerations. Clin Pharmacokinet. 2004;43:963-81.

32. Spyropoulos AC, Turpie AG. Perioperative bridging interruption with heparin for the patient receiving long-term anticoagulation. Curr Opin Pulm Med. 2005;11:373-9. 


\section{Chapter 5}

Effects of peri-operative bridging with low molecular weight heparins on coagulation during interruption of vitamin K antagonists: a mechanistic study 


\begin{abstract}

\section{Background}

Bridging with vitamin $\mathrm{K}$ antagonists (VKA) comprises peri-procedural substitution by low molecular weight heparins (LMWH) upon interruption of VKA. In this exploratory study we investigated the interactive effects of the co-administration of VKA, LMWH and surgery on perioperative coagulation.
\end{abstract}

\title{
Methods
}

Blood was sampled daily from day -3 to day +5 in 13 patients. In addition to measurement of INR and anti-Xa activity, thrombin generation (TG) testing and assessment of individual coagulation factors was performed.

\section{Results}

At the time of intervention the mean INR was 1.0 (SD 0.1, range 0.9-1.2); the mean anti-Xa at day 0 was 0.19 units $/ \mathrm{ml}$ (SD 0.20 units $/ \mathrm{ml}$, range $<0.05-0.60$ ). The intervention caused a 2 to 3 fold increase in TG at day 0 . Of all coagulation factors, factor (F) XI had the strongest correlation with TG ( $r=0.629 ; p=0.02)$ for peak and endogenous thrombin potential (ETP) $(r=0.625 ; p=0.02)$. Thrombomodulin-induced reduction of ETP increased from 10.0\% (SD 9.2) at day -3 to $18.2 \%$ (SD 9.5) at day $0, p=0.02$. After surgery, FVIII and fibrinogen were significantly increased $(p<0.001)$.

\section{Conclusions}

In spite of residual anti-Xa activity at the day of the intervention there is a marked increase in TG related to surgery. Peri-operatively, 3 prothrombotic mechanisms were exposed: FXI dependent TG, reduced activity of the activated protein $C$ pathway and postoperative rises in FVIII and fibrinogen. For the complex perioperative management the value of TG or other global assays to monitor the coagulation balance merits further study. 


\section{Introduction}

Current guidelines advise substitution of longer acting VKA therapy with relatively short acting LMWH in patients at the highest risk of thrombosis in the periprocedural period. ${ }^{1,2}$ Bridging with LMWH therefore is routinely used in patients undergoing invasive procedures that require interruption of VKA, but are deemed too high risk for thromboembolism to allow absence of anticoagulation during the post-intervention period. However, there is only low quality evidence underlying current practice as randomized clinical trials (RCT) were up till recently lacking. The available evidence derived from systematic reviews and meta-analyses suggests that bridging is associated with an increased risk of bleeding. While the bleeding risk may be mostly attributable to the intervention itself, the contribution of fixed, body weight adjusted doses of LMWH in conjunction with stopping/restarting of VKA, may further impair hemostasis in an undesirable manner. ${ }^{3}$ A recent review showed low rates of thromboembolism in bridged and non-bridged patients of respectively 0.9 and 0.6 percent, but associated high rates of bleeding in bridged patients $(13.1 \%$ vs. $3.4 \%$ all bleeds, and $4.2 \%$ vs. $0.9 \%$ major bleeds. ${ }^{4}$ Given the observed elevated risk of bleeding associated with this LMWH bridging policy, the routine application of bridging has been recently challenged. In the very recently published BRIDGE study, in patients randomized to placebo, the risk of thromboembolic events was comparably low to the risk in the LMWH bridged subjects (who had a higher risk of bleeding). Based on these outcomes the authors also discussed the issue of thrombogenicity related to the procedure, stating that "The premise that warfarin interruption leads to rebound hypercoagulability and that the milieu of the procedure confers a prothrombotic state, which in turn leads to arterial thromboembolism, is not supported by the results of this trial". ${ }^{5}$ However, the BRIDGE study included for the large part low risk for thromboembolism patients. Notably, another study showed that there is a 7 times difference in thrombotic risk between more extensive surgery (risks being the highest for hip-surgery or cancer-surgery) compared to less extensive day-surgery risk. ${ }^{6}$ The thromboembolic risks associated with the bridging procedure as well as the underlying mechanisms have not been explored extensively and are therefore largely unknown.

In this explorative study we therefore aimed to investigate the combined effects of cessation of VKA prior to surgery and institution of LMWH in combination with surgery on peri-operative coagulation, assessed by thrombin generation and a panel of its protein determinants. 


\section{Methods}

\section{Patient selection}

During the period from June 2013 until April 201413 patients on chronic treatment with VKA for atrial fibrillation (AF), recurrent venous thromboembolism (VTE), or aortic valve replacement who visited the Maastricht Anticoagulation Clinic, the Netherlands, and in whom bridging of anticoagulation was indicated for planned surgery were invited to participate in the study. Institutional review board approval was obtained (METC 12-1-008). All participants gave informed consent; all patients participated once.

Blood samples were collected daily within a time slot of 3 hours and before noon sharp from day -3 to day +5 , including the day of the intervention. The following inclusion criteria were applied: 1) At least 3 months acenocoumarol use, a VKA that is commonly used in the Netherlands 2) planned invasive procedure 3 ) indication for peri-procedural bridging with LMWH 4) mentally competent, and 5) a minimum age of 18 years. Excluded were 1) patients with severe renal failure (MDRD $<30 \mathrm{ml} / \mathrm{min}$ ), 2) patient who were pregnant or breastfeeding and 3) patients undergoing emergency procedures.

\section{Bridging anticoagulation}

In all patients acenocoumarol was stopped on day -3 , LMWH was installed at day -3 (6 patients) or day-2 (7 patients). The exact bridging regimen was left to the discretion of the prescribing physician, reflecting routine practice. The last dose of LMWH before the intervention was administered at day -1 . Acenocoumarol was re-installed at day 0 (1 patient), day +1 (5 patients) or later ( 7 patients). LMWH was restarted at day 0 (6 patients), day +1 ( 5 patients), day +2 (1 patient), or day +4 (1 patient).

\section{Blood collection and plasma preparation}

Venous blood was collected in $3.2 \%$ citrate $(\mathrm{w} / \mathrm{v})$ citrated vacutainer glass tubes (Becton Dickinson, Plymouth, UK). Within 1 hour after blood collection platelet-poor plasma (PPP) was prepared using two centrifugation steps: the first at 2000xg for 5 minutes and a second step at $10000 x g$ for 10 minutes. Plasma aliquots were snapfrozen, stored at $-80^{\circ} \mathrm{C}$ and thawed at $37^{\circ} \mathrm{C}$ before analysis. The research nurse responsible for the collection of the blood samples recorded the time the sample was taken, the time of LMWH and VKA administration, the type and dose of LMWH and 
VKA, and the time of the intervention (day 0 ) in case report forms (CRF). On day 0 blood was collected before $(n=9)$ or after surgery $(n=4)$.

\section{Thrombin generation measurements}

Thrombin generation in platelet-poor plasma was measured using the Calibrated Automated Thrombogram method (Thrombinoscope BV, Maastricht, The Netherlands), as previously published.[7] The following experimental conditions were used: 0 pM TF with $4 \mu \mathrm{M}$ PL at 20:20:60 mol\% PS:PE:PC, 1 pM TF with $4 \mu \mathrm{M}$ PL in the absence and presence of thrombomodulin (TM) (Thrombinoscope BV) with a final concentration of $0.65 \mathrm{nM}$, and $5 \mathrm{pM}$ TF with $4 \mu \mathrm{M}$ PL. The threshold of detectable thrombin level with this assay is $10 \mathrm{nM}$; therefore we imputed for undetectable peak height $(\mathrm{PH})$ values a value of $5 \mathrm{nM}$, slope $=0$ and endogenous thrombin potential $(E T P)=0.5 * 5 *$ (average value of start tail (time)-lag time (LT) for the concerning reagent).

\section{Measurement of plasma factor levels, INR and anti-Xa}

The INR was assessed using Neoplastine-r (STAGO) reagent. Heparin was assessed by means of the anti-Xa levels using the liquid Anti-Xa (STAGO) and a specific LMWH calibrator; the threshold of detectable anti-Xa level with this assay is $0.05 \mathrm{IU} / \mathrm{ml}$. Plasma fibrinogen levels were measured using the Clauss method using liquid Fib as reagent (STAGO). All other coagulation factor levels were determined by one-stage PT-based (FII, FV, FVII, FX) or aPTT-based (FVIII, FIX, FXI, FXII) clotting assays using plasma deficient in factor $\mathrm{V}, \mathrm{VII}, \mathrm{X}, \mathrm{XI}$, or XII, or plasma immune-depleted for factors VIII, or IX, as reagents. AT levels (FX-a inhibitory activity) with Stachrom AT as reagent and protein $\mathrm{C}$ levels (Stachrom prot $\mathrm{C}$ ) were measured using chromogenic assays. All plasma factor level measurements employed commercially available standards (STAGO, Asnières sur Seine, France) calibrated to WHO standards. All assays were performed on a STA-R coagulation analyzer (STAGO).

\section{Statistical analysis}

Descriptive statistics were used to determine patient and procedure characteristics. Continuous variables are reported as means, and their standard deviations (SD); categorical data are presented as counts and percentages. When related samples were analyzed Wilcoxon signed-ranks or Friedman tests were applied as appropriate. Non-related samples were tested with Mann-Whitney, Kruskal-Wallis or Student's t-tests as appropriate. Pre and post interventional comparisons were made: day -3 to 
day -1 (pre intervention) and day +1 to day +5 (post intervention). To allow linear modeling using linear regression with time expired since LMWH administration as independent variable and anti-Xa levels as dependent variable, anti-Xa values were log transformed (In anti-Xa) and measurements in which blood sample collection occurred within 4 hours after LMWH administration were excluded; because LMWH values peak 4 hours after administration.[8] To account for repeated measurements we constructed linear models for all patients individually and calculated the time from $\mathrm{LMWH}$ administration to an anti-Xa level of $0.20 \mathrm{IU} / \mathrm{ml}$ using these models; the mean values are reported. According to the linear regression model time expired since $\mathrm{LMWH}$ administration to reach an anti-Xa level of $0.20 \mathrm{IU} / \mathrm{ml}$ is expressed as $\left(\mathrm{In} 0.2-\beta_{0}\right.$ (intercept)) $/ \beta_{1}$ ). Correlations were determined using Pearson's correlation coefficient. Mean values of the different variables for each patient were taken into account. A two sided $p$-value $<0.05$ was considered statistically significant. Data were analyzed with SPSS version 22.

Study results are reported according to the recommendations for studies in periprocedural antithrombotic and bridging therapy issued by the ISTH. ${ }^{9}$

\section{Results}

\section{Patient characteristics}

Patient characteristics are presented in Table 5.1. The average age of the patients was 64.7 (SD 9.5), 8/13 (61.5\%) were male. All participants, 13/13 (100\%) were on acenocoumarol treatment for atrial fibrillation (8), DVT (4) and prosthetic aortic valves (1). All patients but one (dalteparin) received therapeutic subcutaneous doses (tinzaparin, nadroparin or enoxaparin) of LMWH peri-operatively: once daily in 12 (92.3\%), and twice daily in 1 (7.7\%). One of thirteen patients $(7.7 \%)$ underwent a low bleeding/ thrombotic risk procedure (skin biopsy) the remaining 12/13 (82.3\%) underwent a high bleeding/thrombotic risk procedure (umbilical hernia repair (2), ablation for AF (2), orthopedic surgery (4), transurethral resection of the prostate (2), cholecystectomy (1), and nephrectomy (1)). 
Table 5.1 Patient characteristics, procedure characteristics, anticoagulation and events

\begin{tabular}{lll}
\hline $\mathrm{N}=13$ & & \\
\hline Age & & $64.7(\mathrm{SD} 9.5)$ \\
Male & & $8(61.5 \%)$ \\
$\mathrm{BMI}\left(\mathrm{kg} / \mathrm{m}^{2}\right)$ & $30.7(\mathrm{SD} 5.2)$ \\
Anticoagulation & & \\
& acenocoumarol & $13(100 \%)$ \\
& nadroparin 7,600 IU(2x) & $1(7.7 \%)$ \\
& tinzaparin 10,000 IU & $2(15.4 \%)$ \\
& tinzaparin 14,000 IU & $4(30.8 \%)$ \\
& tinzaparin 18,000 IU & $3(23.1 \%)$ \\
& tinzaparin 10,000 IU+ 14,000 IU & $1(7.7 \%)$ \\
& enoxaparin 80 mg & $1(7.7 \%)$ \\
& dalteparin 2,500 IU & $1(7.7 \%)$ \\
NSAID/Antiplatelet therapy & \\
& COX inhibitors & $4(30.8 \%)$ \\
& ADP-receptor antagonists & $1(7.7 \%)$ \\
& None & $8(61.5 \%)$ \\
MDRD (ml/min) & & \\
& $>60$ & $8(61.5 \%)$ \\
30-60 & $5(38.5 \%)$ \\
& $<30$ & $0(0 \%)$ \\
\hline
\end{tabular}

Abbreviations: $A F$, atrial fibrillation; BMI, body mass index; IU, international units; MDRD, modification of diet in renal disease; NSAID, non-steroidal anti-inflammatory drug; SD, standard deviation; TE, thromboembolism;

\section{Changes in plasma factor levels, INR, anti Xa from day -3 to day +5}

The mean INR ( $n=13)$ at day -3 (the day acenocoumarol was stopped) of 2.6 (SD 0.9, range 1.1-4.8) normalized to a mean of 1.0 (SD 0.1, range 0.9-1.2) at day 0 (the day of the intervention). At day +5 the INR had increased to a mean of 1.8 (SD 0.8, range 1.0-3.0). The mean residual anti-Xa level at the day of the intervention was $0.19 \mathrm{IU} / \mathrm{ml}$ (SD $0.20 \mathrm{IU} / \mathrm{ml}$, range 0.05- 0.60) See Figure 5.1 for the course of anti-Xa levels and INR; see Table 5.2 for an overview of per patient anti-Xa levels, dose and timing of LMWH administration at day 0.

\section{Elimination of therapeutic doses tinzaparin after the last pre interventional administration}

For all 12 patients who received therapeutic doses of LMWH the time to reach an antiXa level of $0.20 \mathrm{IU} / \mathrm{ml}$ was calculated. For therapeutic doses of LMWH the models for 12 patients combined, predict a mean duration of 22 hours and 52 minutes $(95 \% \mathrm{Cl}$ : $14 \mathrm{~h}, 40 \mathrm{~min}-31 \mathrm{~h}, 20 \mathrm{~min}$ ); the models for all patients individually ranged from $11 \mathrm{~h}$, 
$29 \mathrm{~min}$ to $45 \mathrm{~h}, 32 \mathrm{~min}$. An anti-Xa level of $\geq 0.20 \mathrm{IU} / \mathrm{ml}$ is considered clinically relevant to prevent thrombosis. ${ }^{10}$

Figure 5.1 Mean concentration of plasma factor levels and INR (a) and mean anti-Xa levels (IU/ml) and INR (b) from day -3 to day +5 with their $95 \%$ confidence intervals

a

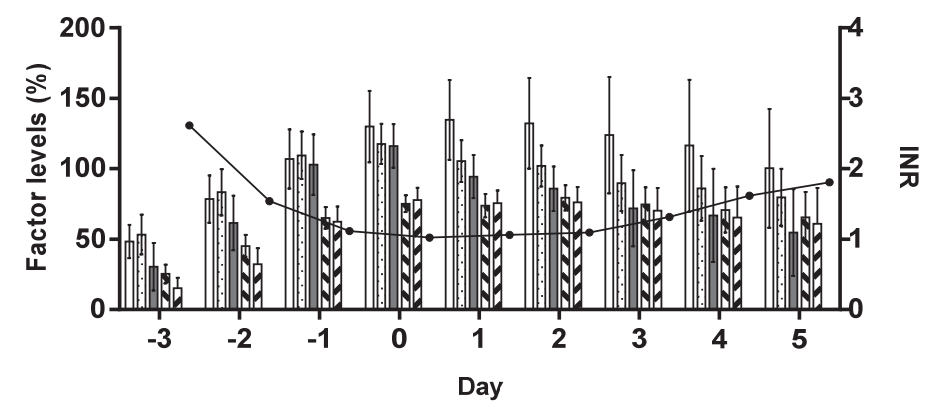

$\square$ FIX

D.... PC

b

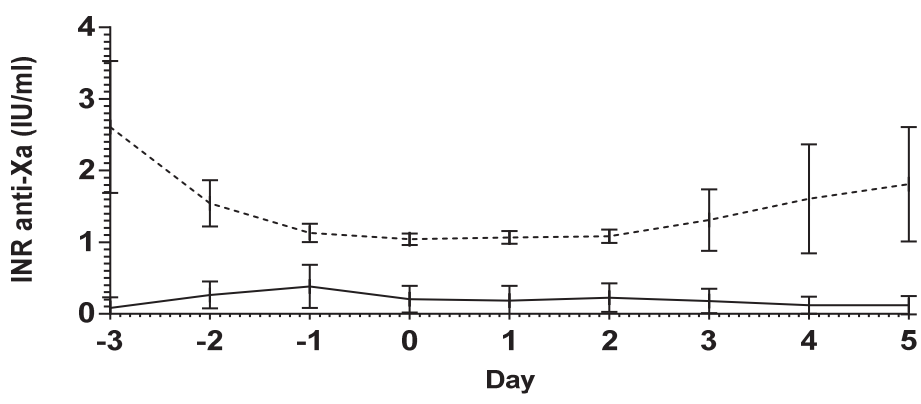

Abbreviations: INR, International Normalized Ratio; PC, Protein C

The effect of the acute phase on plasma factor VIII and fibrinogen levels

We also examined the peri-operative changes in the concentrations of the acute phase reactants FVIII and fibrinogen. The mean pre interventional FVIII and fibrinogen concentrations were $175.9 \%$ (SD 58.9\%) and $4.3 \mathrm{~g} / \mathrm{l}$ (SD $1.1 \mathrm{~g} / \mathrm{l}$ ) respectively; the mean post interventional concentrations were $246.7 \%$ (SD $71.4 \%$ ) and $5.6 \mathrm{~g} / \mathrm{l}$ (SD $1.7 \mathrm{~g} / \mathrm{l}$ ) respectively; for FVIII, $p=0.002$, for fibrinogen $p=0.003$. The mean concentrations of FVIII and fibrinogen on the day of the intervention for the group of patients in whom blood samples were taken prior to surgery were $165.8 \%$ and $4.3 \mathrm{~g} / \mathrm{l}$ respectively; postsurgery concentrations were $234.0 \%$ and $4.5 \mathrm{~g} / \mathrm{l}, \mathrm{p}=0.09$ for FVIII and $p=0.78$ for fibrinogen. 
Effects of peri-operative bridging with LMWH on coagulation during interruption of VKA

Table 5.2 Overview of per patient anti Xa levels, dose and timing of LMWH administration at day 0 , bodyweight, renal clearance and periprocedural bleeding events

\begin{tabular}{clllll}
\hline Patient & $\begin{array}{l}\text { Anti Xa } \\
(\mathrm{IU} / \mathrm{ml})\end{array}$ & $\begin{array}{l}\text { Time between last LMWH } \\
\text { administration and blood sampling }\end{array}$ & $\begin{array}{l}\text { BMI } \\
\left(\mathrm{kg} / \mathrm{m}^{2}\right)\end{array}$ & $\begin{array}{l}\text { MDRD } \\
(\mathrm{ml} / \mathrm{min})\end{array}$ & $\begin{array}{l}\text { Bleeding } \\
(\mathrm{minor} / \mathrm{major})\end{array}$ \\
\hline 1 & $<0.05 \mathrm{IU} / \mathrm{ml}$ & 31 hours, 45 minutes & 36.9 & 43 & No bleeding \\
2 & $0.08 \mathrm{IU} / \mathrm{ml}$ & 22 hours, 15 minutes & 28.9 & $>60$ & $\begin{array}{l}\text { No bleeding } \\
\text { Major }\end{array}$ \\
3 & $0.09 \mathrm{IU} / \mathrm{ml}$ & 25 hours, 15 minutes & 34.4 & 45 & No bleeding \\
4 & $0.26 \mathrm{IU} / \mathrm{ml}$ & 25 hours, 15 minutes & 23.7 & $>60$ & Minor \\
5 & $<0.05 \mathrm{IU} / \mathrm{ml}$ & 25 hours, 45 minutes & 40.7 & $>60$ & No bleeding \\
6 & $0.07 \mathrm{IU} / \mathrm{ml}$ & 26 hours, 45 minutes & 23.2 & $>60$ & Major \\
7 & $0.10 \mathrm{IU} / \mathrm{ml}$ & Unknown & 30.6 & $>60$ & No bleeding \\
8 & $0.13 \mathrm{IU} / \mathrm{ml}$ & 22 hours, 45 minutes & 34.2 & $>60$ & Major \\
9 & $0.13 \mathrm{IU} / \mathrm{ml}$ & 35 hours, 45 minutes & 30.4 & 39 & Major \\
10 & $0.14 \mathrm{IU} / \mathrm{ml}$ & 22 hours, 30 minutes & 23.7 & 34 & No bleeding \\
11 & $0.26 \mathrm{IU} / \mathrm{ml}$ & 21 hours, 45 minutes & 32.1 & $>60$ & Minor \\
12 & $0.58 \mathrm{IU} / \mathrm{ml}$ & 14 hours, 0 minutes & 30.4 & $>60$ & minor \\
13 & $0.60 \mathrm{IU} / \mathrm{ml}$ & 16 hours, 30 minutes & 30.1 & 42 & \\
\hline
\end{tabular}

Abbreviations: IU, international units; LMWH, low molecular weight heparin; MDRD, modification of diet in renal disease

\section{Thrombin generation from day -3 to +5}

In Figure 5.2 an overview of ETP and PH triggered with respectively 1pM of TF and $5 \mathrm{pM}$ of TF with and without the addition of TM is presented per day and Table 5.3 offers an overview of all TG parameters per day. The mean ETP 1pm TM at day -3 was $292.3 \mathrm{nM}^{*} \mathrm{~min}$, then peaked at day 0 to $1003.5 \mathrm{nM} * \min$ (in the group of patients in whom blood collection was performed after the intervention), then gradually dropped; day +2 (756.5 nM * min), day +5 (482.3 nM * min). PH 1 pM showed a similar pattern: day $-354.0 \mathrm{nM}$, day $0207.2 \mathrm{nM}$ (in the group of patients in whom blood collection was performed after the intervention), day $+2141.3 \mathrm{nM}$, day +5 84.6 $\mathrm{nM}$. The mean values of ETP $5 \mathrm{pM}$ and PH 5 pM showed the same pattern.

The correlations between the mean per patient concentration of prothrombin, FV, FVII, FVIII, FIX, FX, FXI, FXII, PC, AT, fibrinogen and different parameters of thrombin generation triggered by all 3 reagents were calculated. Predominantly, weak to moderate, non-significant correlations were observed. Several correlations between FVIII, FXI, and different parameters (all triggers) were all strong and significant $(p<0.05)$; Table 5.4. 


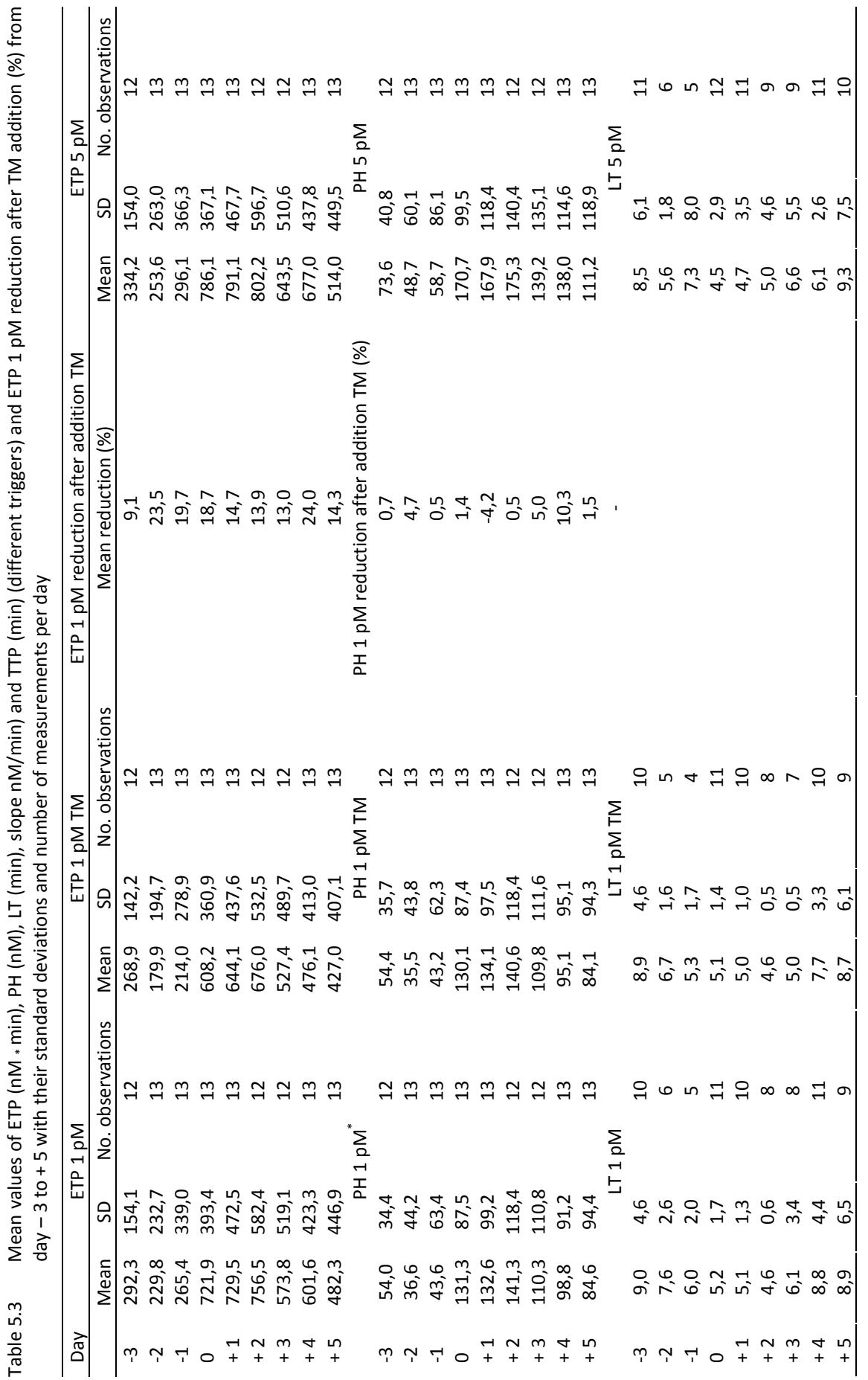


Effects of peri-operative bridging with LMWH on coagulation during interruption of VKA

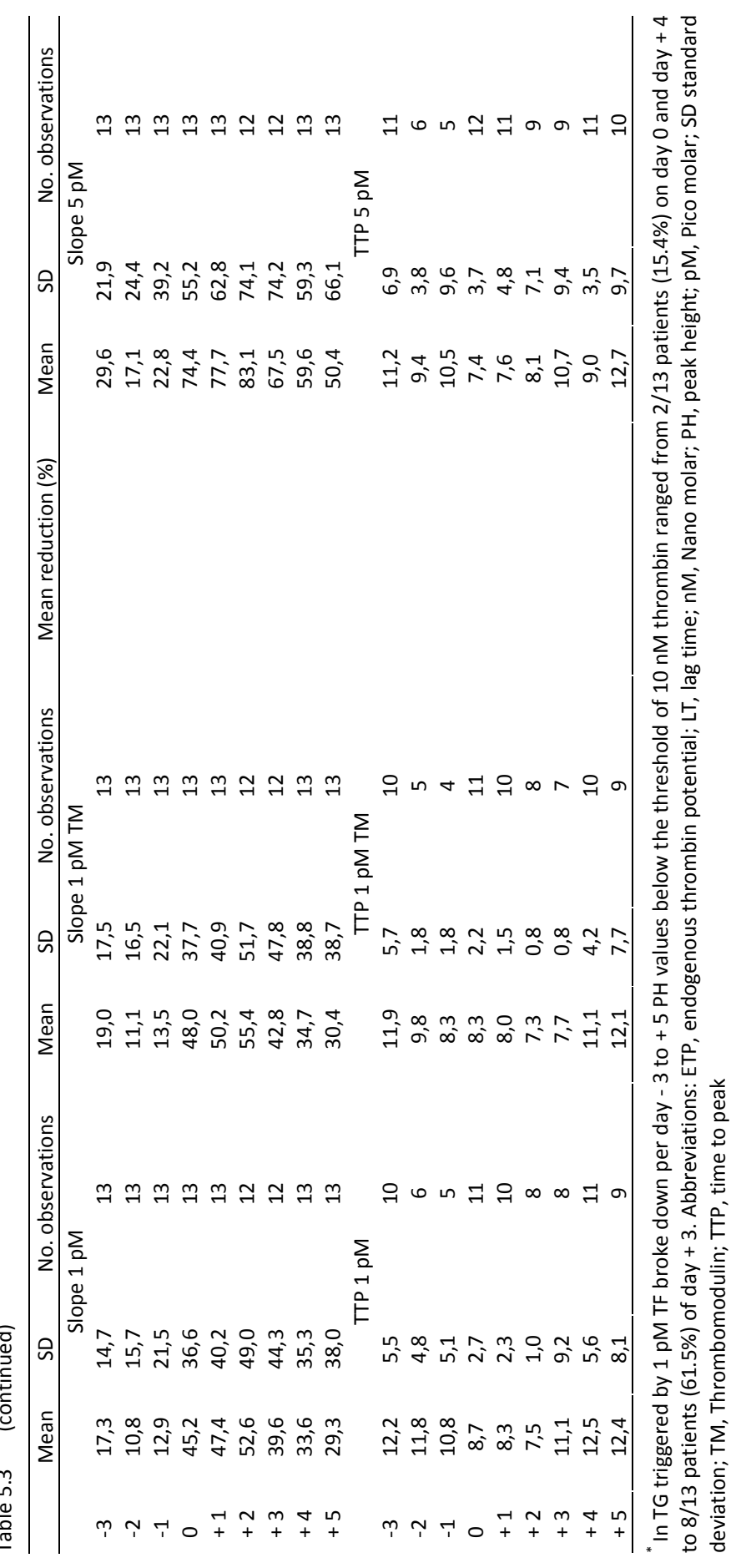


Figure 5.2 Mean endogenous thrombin potential (a) and peak height (b) from day -3 to day +5 with their $95 \%$ confidence intervals

a

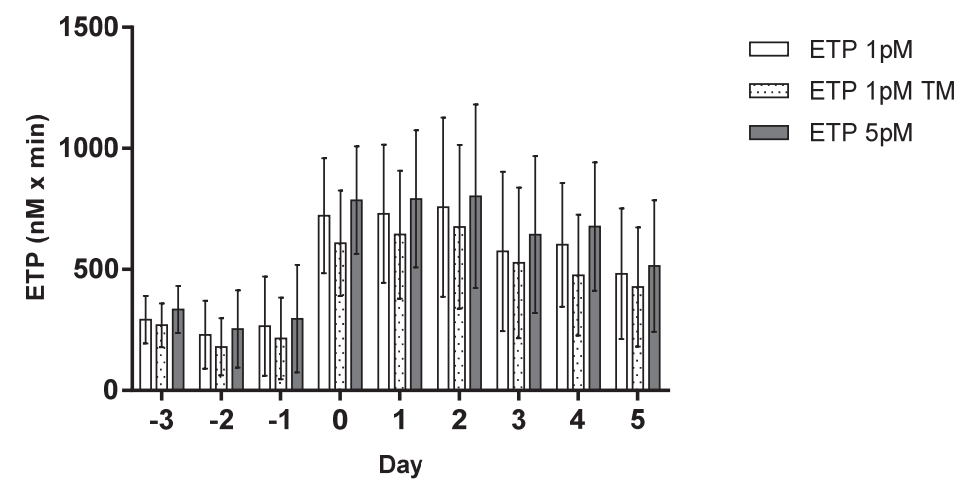

b

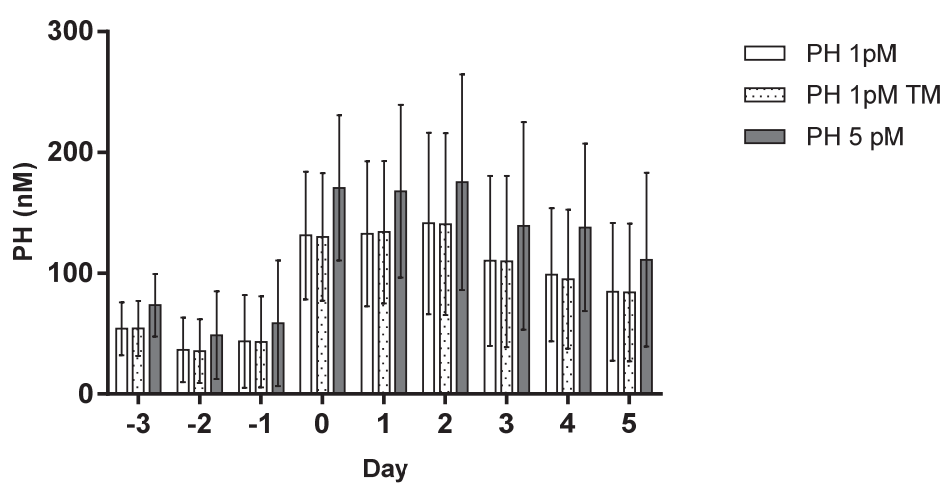

Abbreviations: ETP, endogenous thrombin potential; PH, peak height; pM, Pico molar; TM, Thrombomodulin

To investigate activated PC (APC) resistance, caused by depletion of functional protein $C$ due to VKA use, we compared the decrease in percentage of ETP 1 pM TF caused by the addition of TM for the days with the lowest and highest mean INR; the days in which APC resistance is expected to be minimal and maximal respectively. We computed the mean values of the variable ((ETP 1 pM TF - ETP 1 pM TF + TM) / ETP 1 pM TF) $\times 100$, for all participants on day 0 (day with the lowest mean INR) and day -3 (day with the highest mean INR). The mean values were 9.2 (SD 9.2)\% and 18.6 (SD 8.8)\% for day -3 and day 0 respectively. The Wilcoxon signed-ranks test resulted in a p-value of 0.002. Additional assessment of correlations between mean per patient clotting factor levels and reduction in ETP 1 pM TF did not yield any significant results (Table 5.4). 


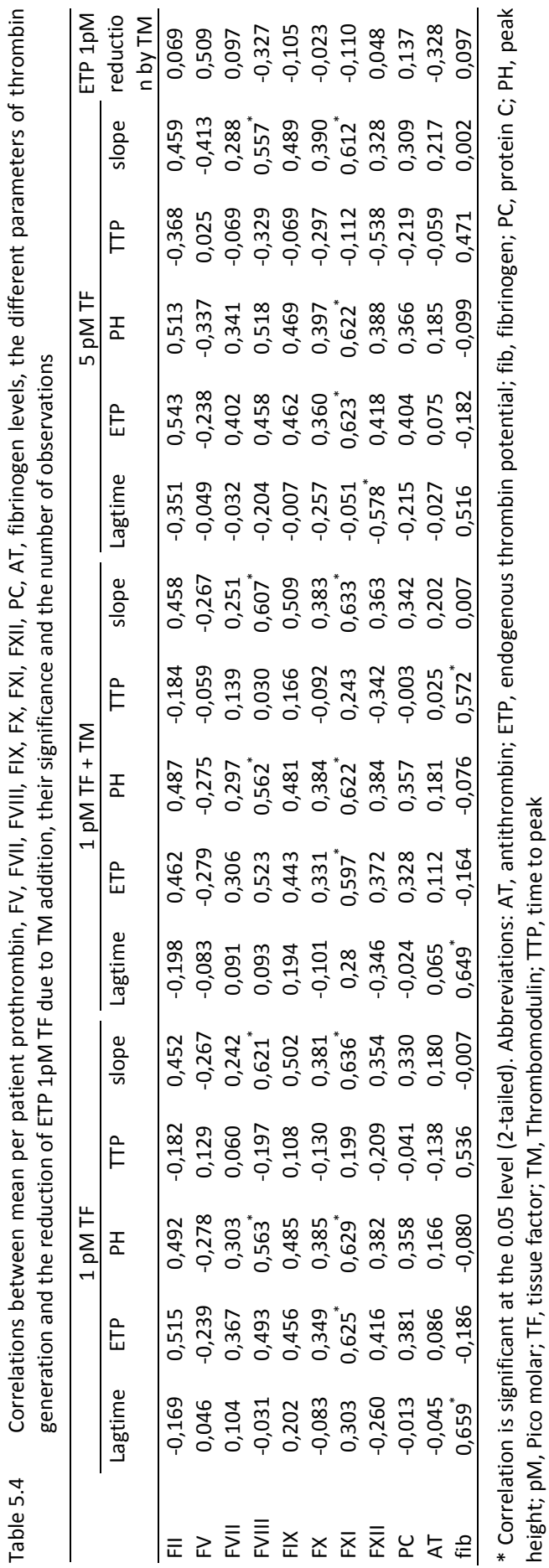




\section{Correlations between INR, anti-Xa, and parameters of thrombin generation}

Correlations between mean INR per patient and mean per patient plasma levels of FII, FVII, FIX, FX and protein C were negative, strong, and significant for FII, FVII and protein $\mathrm{C}$. Bonferroni corrected significance of 0.01 was reached for all factors, except the correlations INR-FIX ( $r=-0.36, p=0.22)$ and INR-FX ( $r=-0.65, p=0.02)$ (Table 5.5). Correlations between INR and different parameters of thrombin generation triggered by $1 \mathrm{pM}$ TF with and without the addition of TM, and triggered by $5 \mathrm{pM}$ were moderate and significant $(\mathrm{p}<0.05)$ for LT (all triggers), and for TTP 1 PM TF+TM and TTP 5 pM TF; weak, inverse correlations were observed between INR and slope, PH and ETP. For comparison we also calculated the correlations between INR and TG on days no LMWH was administered; overall we found similar correlations, p-values $<0.05$ were reached for LT (all triggers) (Table 5.5).

Table 5.5 Correlations between mean per patient INR, anti-Xa and mean per patient factor levels, different parameters of thrombin generation, their significance and the number of observations

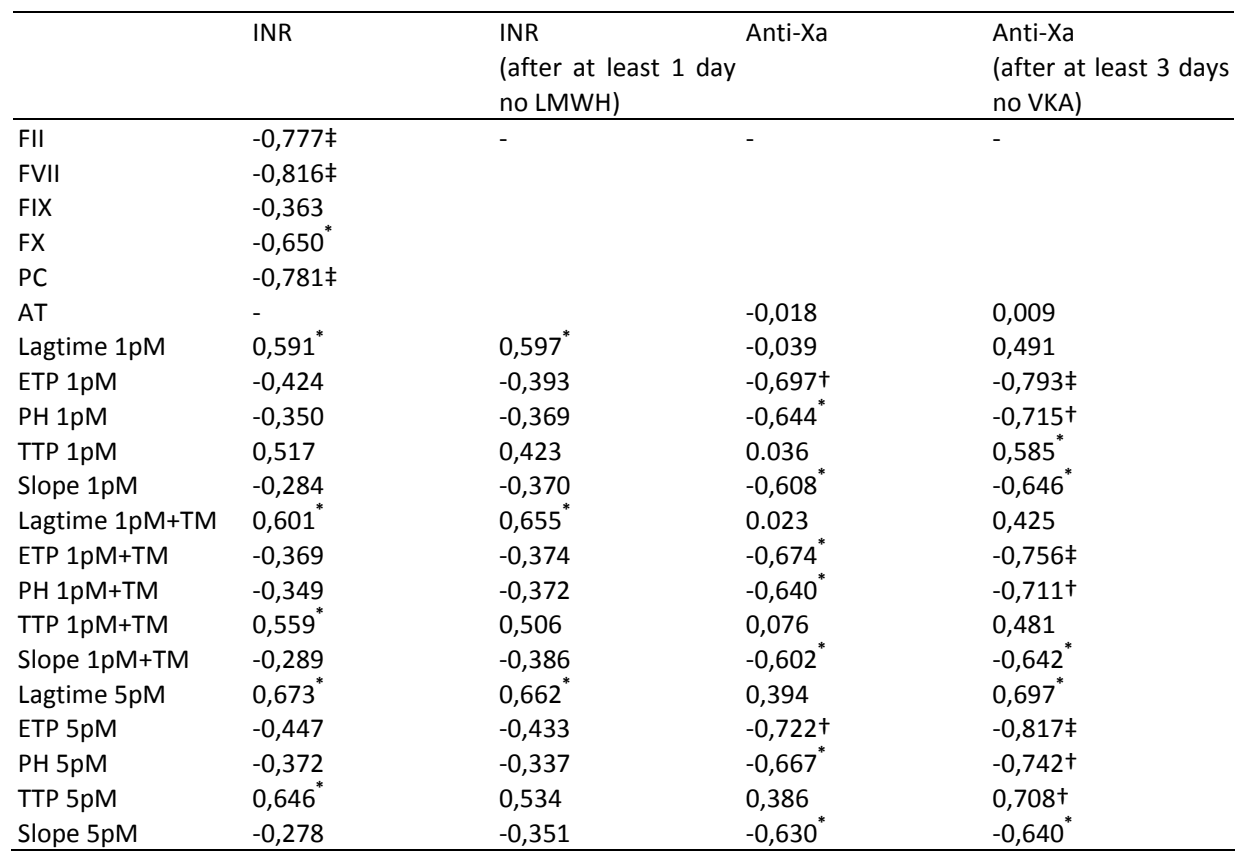

* Correlation is significant at the 0.05 level (2-tailed); + Correlation is significant at the 0.01 level (2-tailed); $¥$ Correlation is significant at the 0.003 level (2-tailed). Abbreviations: ETP, endogenous thrombin potential; INR, International Normalized Ratio; PH, peak height; pM, Pico molar; TM, Thrombomodulin; TTP, time to peak 
Correlations between the mean anti-Xa per patient and different parameters of thrombin generation triggered by all 3 reagents were calculated. A strong correlation between ETP (all triggers) was found, ETP 1 pM TF and ETP 5 pM TF reached a p-value of $<0.01$. Other TG parameters showed moderate correlations, a significance level of $<0.05$ was reached for 9/15 TG parameters. No measurement reached the Bonferroni corrected $p$-value $<0.003$. Overall stronger correlations were observed between the mean per patient anti-Xa and the parameters of TG when only the days that no acenocoumarol was administered for at least 3 days were included in the analysis. The observed correlations for ETP (all triggers) were stronger and Bonferroni corrected $p$ values $<0.003$ were recorded for all three ETP triggers. Compared to the correlations that ignored VKA activity higher levels of correlation were also found for LT and PH, slope and ETP (all triggers). A significance level of $<0.05$ was reached for $12 / 15$ TG parameters (Table 5.5).

\section{Post procedural bleeding and thromboembolism}

Our study was explorative in character and not powered to associate outcomes to levels of individual coagulation factors or levels of anticoagulants. No TE (stroke, TIA, systemic embolism) was reported in any patient. However, we observed 4 major bleeding events (30.8\%) and 3 minor bleeding events (23.1\%) during the 30 -day post procedural follow-up; all patients recovered. During the time of the bleeding event all patients had INRs close to 1 (1.0-1.1), but 3 of the patients had residual anti Xa levels of $>0.10 \mathrm{IU} / \mathrm{ml}$ and 3 patients had impaired kidney function (MDRD $34 \mathrm{ml} / \mathrm{min}, 39$ $\mathrm{ml} / \mathrm{min}$, and $45 \mathrm{ml} / \mathrm{min}$ respectively). ETP at $1 \mathrm{pM} \mathrm{TF} \mathrm{371,4} \mathrm{nM}^{*} \min$ (SD 196.7) and $\mathrm{PH}$ at $1 \mathrm{pM}$ TF $65,8 \mathrm{nM}$ (SD 41,7) were lower than those of patients without major bleeding; ETP at 1 pM TF 588,5 nM*min (SD 215,0) and PH at 1 pM TF 105,9 nM (SD $50,9)$, but these differences were not statistically significant $(p=0,11$ and 0,20 respectively). Similar results were obtained with 5 pM TF activation.

\section{Discussion}

Surprisingly, the recently published BRIDGE trial suggests that peri-operative bridging with LMWH would no longer be needed, at least in patients undergoing minor surgical interventions, since the risk of thromboembolism was comparable to the rate observed in non-bridged control patients. ${ }^{5}$ This raises the question whether brief cessation of VKA is associated with any rebound hypercoagulability due to surgery. In order to assess peri-operative coagulation activity in patients undergoing surgical 
interventions, we determined the overall impact of cessation of VKA, peri-procedural instillation of LMWH and surgery on net coagulation activity, measured by TG. To understand the nature of any changes in TG, we measured all relevant coagulation proteins that may determine the TG profile.

The results of our exploratory study shows that surgical intervention quickly induced a marked increment in TG peak and ETP values, confirming a "rebound" prothrombotic state. In a further analysis of coagulation proteins we observed that FVIII and FX, but in particular FXI had significant effects on the rate and height of TG. The effect of FXI may support the apparent importance of this protein in driving the risk of postoperative thromboembolism, as illustrated by a recent trial showing better efficacy of FXI inhibition as compared to LMWH. ${ }^{11}$ Release of tissue factor may engage increased thrombin generation, which through a feedback loop amplifies FXI dependent TG. In this process the contributions of FVIII, FIX and prothrombin are also evident; FVIII was in this setting observed to be a determinant for thrombin generation. Interestingly, the rise in FVIII and fibrinogen occurs gradually, due to the well-known cytokine dependent, acute phase synthesis and the contribution of FVIII to TG may therefore only become evident days after the intervention. Several studies report increased levels of F VIII and fibrinogen until 2 days to 72 hours postoperatively. ${ }^{12,13}$ This may in part explain the protracted risk of thrombosis following surgery. The residual anticoagulant activity may in part be neutralized by the acute phase thrombogenic response following the intervention. Another contributor to the thrombosis risk may be the reduced sensitivity to activated protein C (APC resistance) mimicked in our experiments by adding TM to plasma. While paradoxically, during VKA treatment due to the reduced level of PC, this prothrombotic APC resistance effect is already evident, it does not fully correct when the VKA effect is no longer present as indicated by INR of 1.0 at day 0 . The TM reduction is optimized to give a $50 \%$ reduction in $\mathrm{PH}$ of a normal plasma, but the maximum reduction observed in these patients was around $20 \%$; since PC had at that stage reached normal values, this suggests that other factors contribute to the apparent TM related APC resistance, adding to the risk of thrombosis. Theoretically, the unexplained but steady increase in FV levels may have attributed to this APC resistance effect.

Cessation of VKA and start of LMWH induces marked changes in the coagulation proteome. Strong, inverse correlations between INR and vitamin $\mathrm{K}$ dependent pro and anticoagulants were observed. Weak to moderate correlations between INR and TG parameters were recorded; on the other hand, correlations between anti-Xa levels and TG were moderate to strong and significant. The correlations between anti-Xa and TG were stronger after correction for the effect of VKA. In a study in which the 
concomitant effect of VKA (in vivo administration) and LMWH (added in vitro) on different parameters of TG was investigated, similar findings were reported. ${ }^{14}$ It is possible that the effect on TG of LMWH in combination with AT, which leads to inactivation of FXa and thrombin and subsequent release of TFPI from the endothelium, forming a complex with FXa, TF and FVIIa, is stronger than the effect of the inhibition of prothrombin, FVII, FIX and FX by VKA.

The current study was set up as a mechanistic study, this could be considered as a limitation as it restricts the potential for the assessment of significant risk associations. We did however even within this limited sample observe 4 cases of major bleeding. Three out of 4 had reduced renal function and anti Xa levels $>0.1 \mathrm{IU} / \mathrm{ml}$ suggesting that bridging with $\mathrm{LMWH}$, especially in patients with renal impairment should be monitored closely. TG tended to be lower in patients who experienced bleeding; no statistically significant difference in levels was observed, most likely due to the limited sample size.

Our study has several strengths. In the first place, as far as we know this is the first study that evaluates bridging therapy by means of a wide range of blood coagulation assays. Results of this exploratory study might offer insight in the so far poorly understood effects of the complex in vivo interaction between VKA and LMWH administration, and the effects of surgery on hemostasis. Secondly, we were able to follow the patients for a 9-day period, so that the whole perioperative bridging period could be evaluated.

We conclude that VKA (acenocoumarol) arrest in bridged patients starting 3 days before surgery gives an effective reduction of INR, but that LMWH may need to be individually tailored. We found that for several patients the ACCP advised timeframe of 24 hours between the last preoperative therapeutic dose administration of LMWH and planned surgery is not long enough to warrant sufficient decline in anti-Xa levels. In spite of residual anti-Xa activity, there was a marked increase in TG related to surgery. Three prothrombotic mechanisms are exposed: FXI dependent TG, reduced activity of the APC pathway and postoperative rise in FVIII and fibrinogen. For the complex perioperative hemostasis management the value of TG or other global assays, to monitor the hemostatic balance, therefore merits further study. 


\section{References}

1. Douketis JD, Spyropoulos AC, Spencer FA, et al. Perioperative management of antithrombotic therapy: Antithrombotic Therapy and Prevention of Thrombosis, 9th ed: American College of Chest Physicians Evidence-Based Clinical Practice Guidelines. Chest 2012;141:326-50.

2. Keeling D, Baglin T, Tait C, et al. British Committee for Standards in $H$. Guidelines on oral anticoagulation with warfarin - fourth edition. Br J Haematol 2011;154:311-24.

3. Eijgenraam P, Ten Cate H, Ten Cate-Hoek AJ. Safety and Efficacy of Bridging with low molecular weight heparins: a systematic review and partial meta-analysis. Curr Pharm Des 2012;19:4014-23.

4. Siegal D, Yudin J, Kaatz S, Douketis JD, et al. Periprocedural heparin bridging in patients receiving vitamin $\mathrm{K}$ antagonists: systematic review and meta-analysis of bleeding and thromboembolic rates. Circulation 2012;126:1630-9.

5. Douketis JD, Spyropoulos AC, Kaatz S, et al. Perioperative bridging anticoagulation in patients with atrial fibrillation. N Engl J Med. 2015;373:823-33.

6. Sweetland S, Green J, Liu B, et al. Duration and magnitude of the postoperative risk of venous thromboembolism in middle aged women: prospective cohort study. BMJ 2009;339:4575-83.

7. Dielis AW, Castoldi E, Spronk HM, et al. Coagulation factors and the protein C system as determinants of thrombin generation in a normal population. J Thromb Haemost 2008;6:125-31.

8. Garcia DA, Baglin TP, Weitz Jl, et al. Parenteral anticoagulants: antithrombotic therapy and prevention of thrombosis, 9th ed: American College of Chest Physicians Evidence-Based Clinical Practice Guidelines. Chest 2012;141:24-43.

9. Spyropoulos AC, Douketis JD, Gerotziafas G, et al. Periprocedural antithrombotic and bridging therapy: recommendations for standardized reporting in patients with arterial indications for chronic oral anticoagulant therapy. J Thromb Haemost 2012;10:692-4.

10. Harder S, Klinkhardt U, Alvarez JM. Avoidance of bleeding during surgery in patients receiving anticoagulant and/or antiplatelet therapy: pharmacokinetic and pharmacodynamic considerations. Clin Pharmacokinet 2004;43:963-81.

11. Buller HR, Bethune $\mathrm{C}$, Bhanot $\mathrm{S}$, et al. Factor $\mathrm{XI}$ antisense oligonucleotide for prevention of venous thrombosis. N Engl J Med 2015;372:232-40.

12. Oberweis BS, Cuff G, Rosenberg A, et al. Platelet aggregation and coagulation factors in orthopedic surgery. J Thromb Thrombolysis 2014;38:430-8.

13. Nygaard OP, Unneberg K, Reikeras O, et al. Thromboplastin activity of blood monocytes after total hip replacement. Scand J Clin Lab Invest 1990;50:183-6.

14. Gerotziafas GT, Dupont C, Spyropoulos AC, et al. Differential inhibition of thrombin generation by vitamin $\mathrm{K}$ antagonists alone and associated with low-molecular-weight heparin. Thromb Haemost 2009;102:42-8. 


\section{Chapter 6}

Venous stenting after deep venous thrombosis and antithrombotic therapy: a systematic review

Pieter Eijgenraam, Hugo ten Cate, Arina J ten Cate-Hoek

Reviews in Vacular Medicine. 2014;2:88-97 


\begin{abstract}
Introduction

Over the last years venous stent placement after deep venous thrombosis (DVT) in the iliofemoral veins has gained more attention. The majority of studies evaluating the safety and efficacy of this intervention are of poor methodological quality and the association with antithrombotic therapy has not been studied explicitly. We performed a systematic review to summarize the available literature on antithrombotic management in relation to the safety and efficacy of venous stenting.
\end{abstract}

\title{
Methods
}

We performed a Medline search to identify studies that addressed anticoagulation and/or antiplatelet treatment options after venous stenting in patients with a prior DVT in the iliofemoral area. We identified 192 articles and finally selected 14 articles for use in this review.

\section{Results}

In $86 \%(12 / 14)$ of the included studies anticoagulation was administered to all patients who underwent iliac venous stenting. In $33 \%$ of the studies patients received antiplatelet therapy consisting of aspirin and/or clopidogrel (4/12). The duration of antithrombotic treatment was not guided by the stenting procedure in $93 \%(13 / 14)$ of studies. The incidence of re-thrombosis in (sub) groups of only stented patients, ranged from $5 \%$ to $25 \%$. Primary, assisted primary, and secondary patency rates 12 months after stent placement ranged from 54\%, 72\%, 83\% respectively to $78 \%, 83 \%, 95 \%$ in (sub)groups of only stented patients. Rates of major bleedings during long term follow-up ranged from $0 \%$ to $11 \%$.

\section{Conclusion}

Antithrombotic therapy does not seem to influence any of the outcomes in patients with venous stenting after DVT: recurrent DVT, patency, post-thrombotic syndrome or restenosis and bleeding. 


\section{Introduction}

For a substantial proportion of patients with deep venous thrombosis (DVT), current treatment strategies are suboptimal. Especially for the group of patients who are at the highest risk for post-thrombotic syndrome (PTS), new treatment modalities are being investigated. Over the last years the use of stenting of the iliofemoral veins has gained more attention. Nowadays, stenting is predominantly used in patients with venous outflow obstructions of the iliac and femoral veins after thrombus removal with catheter directed thrombolysis (CDT) combined with percutaneous mechanical thrombectomy (PMT). ${ }^{1}$

Arterial interventions are much better studied than venous interventions and the mechanisms of arterial stent failure are therefore better understood. Iliofemoral venous re-stenosis after stenting probably results from intraluminal fibrosis in postthrombotic lesions or compression by primary obstructions, while arterial lesions arise from smooth muscle cell proliferation driven by cytokines and growth factors derived from platelets and macrophages. ${ }^{2}$ Platelet adhesion and aggregation likely plays a more important role in high-flow, high shear conditions in the arterial system, coagulation seems more important in the low-flow, low-shear conditions of the venous system. ${ }^{2,3}$ Finally, both in venous injury and atherosclerotic plaque rupture, the exposure of procoagulant tissue factor and other proteins to blood, triggers blood coagulation. ${ }^{3}$ Therefore the selection of appropriate antithrombotic management is important.

The efficacy and safety profile of venous stenting is evaluated in several clinical studies, but not much attention is given to the optimal duration for the post intervention antithrombotic management. ${ }^{4-20}$ In patients receiving venous stents after a DVT, anticoagulation and possibly antiplatelet therapy may play an important role in preventing recurrent thrombosis and subsequent embolization into the vasculature of the lungs. So far, the choice of agent, dosing and duration of anticoagulant and possibly antiplatelet therapy is unclear for patients with venous stents and prior DVT. The lack of methodologically well-designed studies to address this matter is eminent. While conventional anticoagulants such as vitamin $\mathrm{K}$ antagonists and low molecular weight heparins have proven to reduce the risk of recurrent DVT dramatically, their role in preventing DVT in patients with venous stents remains unclear. Antiplatelet therapy has proven to be safe and efficacious in patients with arterial stents, but the effect in patients with venous stents, with or without prior DVT also remains unclear. The most recently issued ACCP guidelines (2012) do not advise on extended anticoagulation/antiplatelet therapy after venous stenting; the possibility of stenting after thrombolysis in patients presenting with DVT is not even discussed..$^{21}$ Likewise, 
the most recent NICE and ESC guidelines do not discuss venous stenting as a treatment option for patients after a DVT.

Initially we intended to compare the effect of different antithrombotic agents and treatment durations in patients undergoing venous stenting in the iliofemoral area after a first or recurrent DVT, in terms of recurrent DVT, pulmonary embolism (PE), PTS, patency rates and bleeding risk. Since, after our literature search no comparison data were available, we decided to describe and summarize the available literature on different antithrombotic options in relation to these main outcome measures.

\section{Methods}

\section{Study selection}

Research questions and inclusion criteria of the studies were specified in advance and documented in a protocol. The population of this review consists of patients who underwent venous stenting after venous thrombosis, often in combination with balloon angioplasty preceded by CDT and/or PMT. We only included studies wherein the use of post-interventional antithrombotic therapy was addressed. Antithrombotic therapy consist of: 1 ) The administration of anticoagulants (vitamin $\mathrm{K}$ antagonists, unfractionated heparin (UFH), fondaparinux, low molecular weight heparin (LMWH), direct factor Xa inhibitors, direct thrombin (factor Ila) inhibitors, 2) the administration of antiplatelet drugs: cyclooxygenase (COX) inhibitors: aspirin, ADP-receptor antagonists such as clopidogrel, plasugrel and ticagrelor. We decided to also include studies in which not all participants were stented; a part of the population underwent CDT and/or PMT without stenting. Most studies assessed, with the use of duplex, long-term primary patency rates (i.e. patent stent without re-intervention), assisted primary patency rates (i.e. patent stent after re-intervention without any occlusions) and secondary patency rates (i.e. patent stent after occlusion, with patency ending at the moment the occlusion was present). Other reported outcomes were PTS, recurrent DVT, PE and major bleeding complications. Assessment of PTS was performed with different methods, including the Villalta scale and Venous Clinical Severity scores. No restrictions regarding follow-up period were imposed. In this systematic review case reports, cohort studies, case-control studies, cross-sectional studies and randomized controlled trials were eligible. 


\section{Data sources and searches}

We searched MEDLINE database up to week 45 of 2013. The search strategy can be found in appendix 6.1. We used MeSH headings for keyword and text word searching and free text for word searching only. No publication date restrictions were imposed; we restricted our search to articles in English. Further selection was made on title and abstract. The final selection of articles was made after full reading.

\section{Data extraction and quality assessment}

As far as we know no quality assessment tools designed for specific use in noncomparator, observational studies are available. ${ }^{22}$ For the quality assessment of individual studies we made use of the Newcastle-Ottawa Scale (NOS); this tool is designed for cohort studies. ${ }^{23}$ We selected 5 relevant items and prioritized them in our quality assessment (see appendix 6.2). Each item can be awarded with a star, resulting in a maximum of 5 stars. The following items were assessed: representativeness of the cohort, ascertainment of the exposure, assessment of the outcome, length and adequacy of the follow-up. Data collected from individual studies were reported on case report forms consisting of 3 sections: study eligibility (i.e. type of intervention and outcome), checklist of items for data collection (i.e. characteristics of the participants and study design), and quality assessment using the adapted NOS scale for cohort studies. ${ }^{23}$ Data extraction was performed by one reviewer (PE), supervised by the second reviewer (AtC).

\section{Data analysis}

Patency rates in most of the included studies were estimated using the method as proposed by Kaplan-Meier. Differences between groups were assessed using the Student's $t$-test; other outcomes were presented as means with their standard deviation or as percentages. Due to the lack of comparator studies in our review no meta-analysis could be performed.

\section{Results}

\section{Inclusion and quality assessment}

Our MEDLINE database search resulted in a total of 192 studies. For a summary of the search see Figure 6.1. A first selection on title and abstract yielded a total of 27 articles, further restriction after full reading resulted in a set of 14 studies. We identified 12 cohort studies ( 6 prospective, ${ }^{4-7,9,10} 4$ retrospective studies, ${ }^{8,11,14,17}$ 
2 studies with an unclear character), ${ }^{13,18} 1$ trial $^{15}$ and 1 case report. ${ }^{12}$ In only 1 cohort study a comparison with another treatment modality was made; stenting was compared to anticoagulation alone. ${ }^{4}$ We included 3 studies in which the full population was stented; ${ }^{8,13,17}$ one additional study allowed full subgroup analysis of the patients that underwent stenting. ${ }^{11}$ In 10 studies between $15 \%{ }^{15}$ and $89 \%{ }^{11}$ of the population was stented. In all included studies the patients underwent CDT and/or PMT and/or angioplasty and stenting and were prescribed to anticoagulation and/or antiplatelet agents and/or graded compression stockings. For a summary of the included studies see Table 6.1.

Figure 6.1 Summary of evidence search and selection

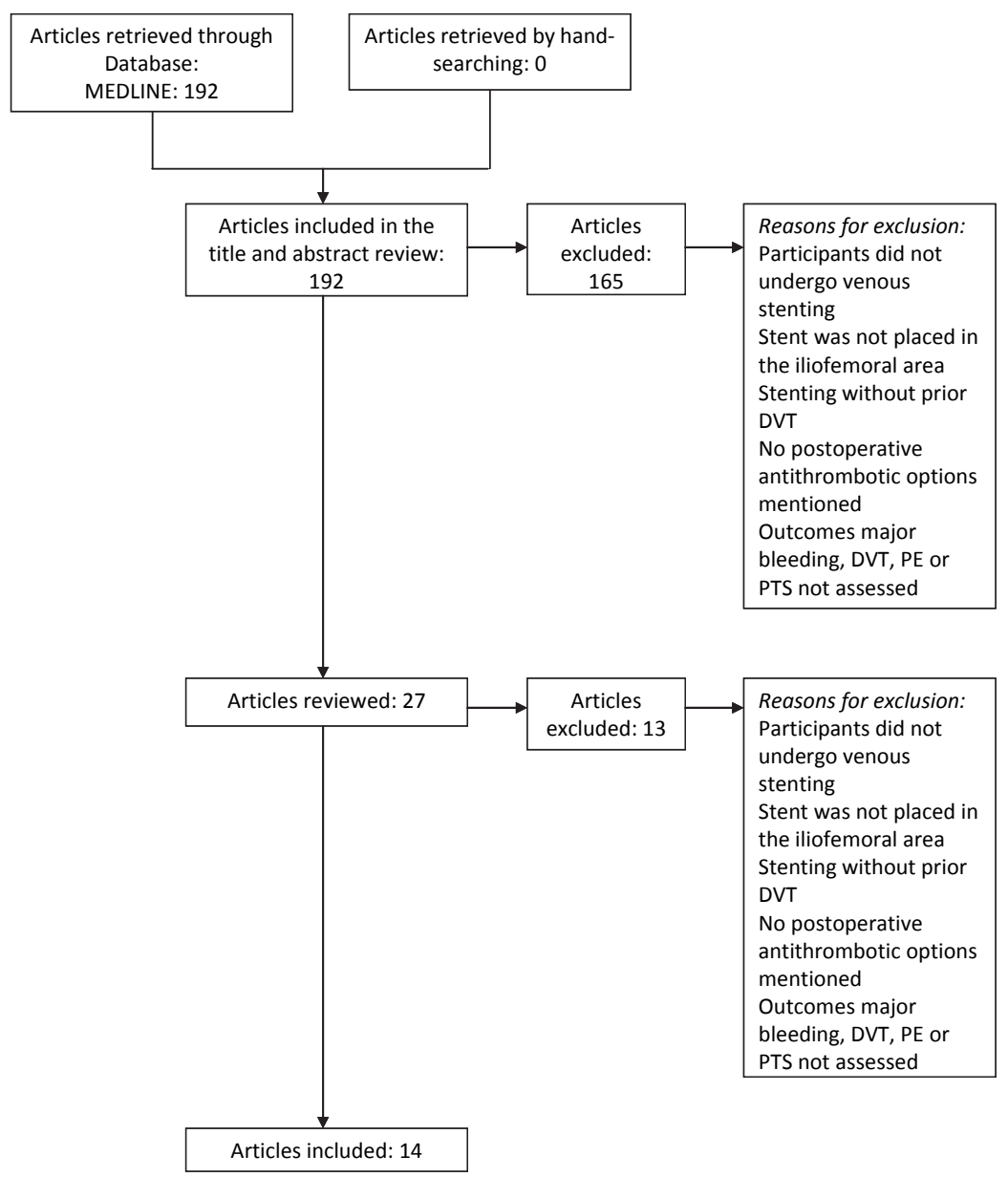

Abbreviations: DVT, deep venous thrombosis; PE, pulmonary embolism; PTS, post thrombotic syndrome 


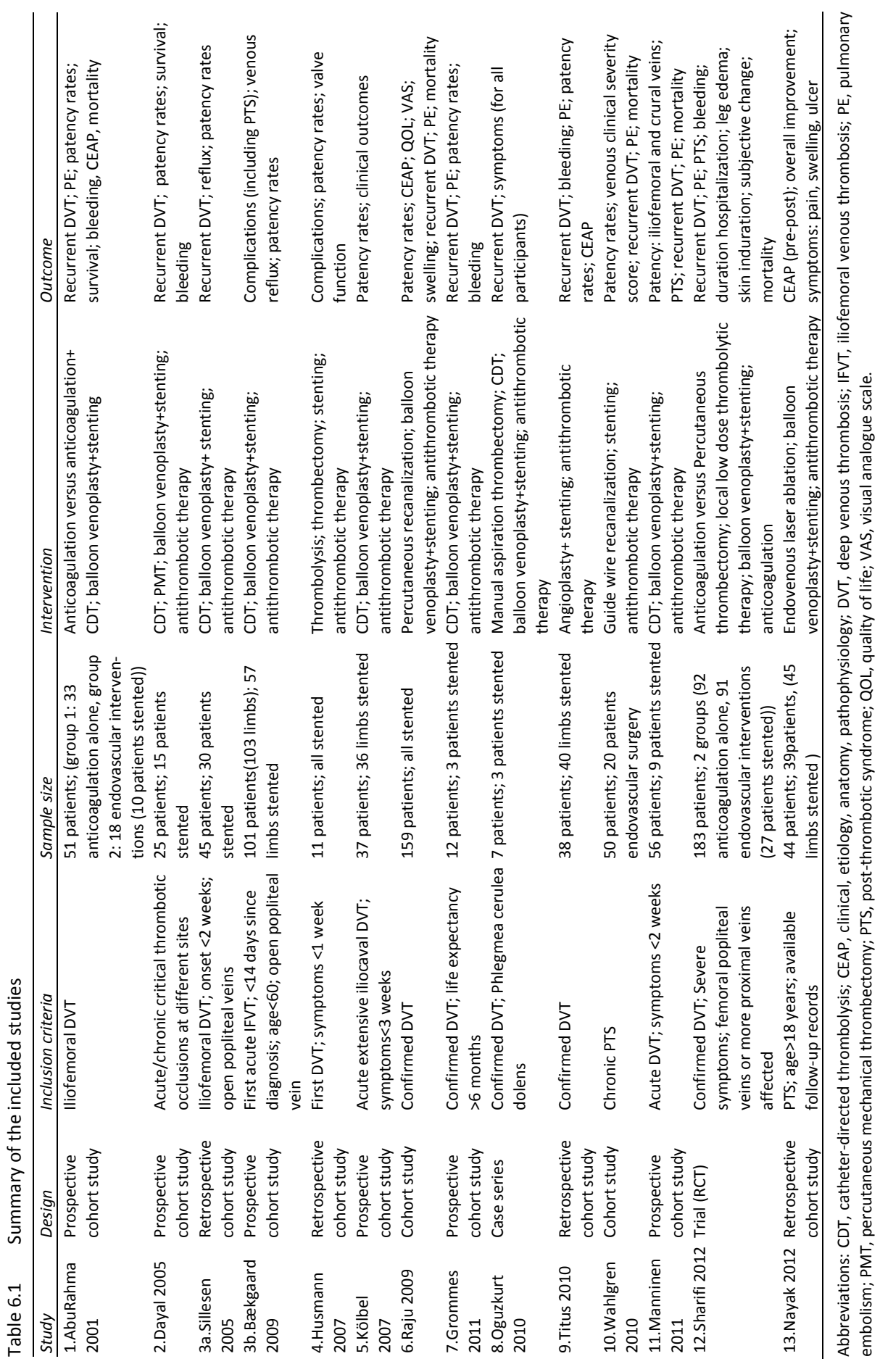


One study was excluded from quality assessment; a case report. ${ }^{12}$ Two studies were awarded with the maximum score of 5 stars. ${ }^{9,15}$ The mean follow-up of the different studies ranged from 7 months $^{7}$ to 5 years. ${ }^{4}$ There were 3 studies with a complete follow-up: 1 retrospective ${ }^{8}$ and 2 small prospective studies; ${ }^{6,9} 3$ studies did not provide any statement on lost of follow up. ${ }^{5,7,13}$ In Table 6.2 a summary of potential sources of bias in the individual studies is presented. NOS scores are reported in the last column. All the included studies reported data on anticoagulant therapy, antiplatelet therapy or the use of compression stockings.

\section{General study characteristics}

In $86 \%$ of the studies only patients with prior DVT (12/14 studies) were included; 2 studies included patients with occlusions due to DVT or external compression. ${ }^{6,17}$ In $57 \%$ of the studies (8/14) only patients with acute DVT were included; ${ }^{4,5,8-10,12,14,15}$ both participants after an acute or chronic DVT were assessed in $36 \%$ of the studies $(5 / 14){ }^{6,7,11,13,17}$ in 1 study only patients with chronic DVT were assessed. ${ }^{18}$ The age in the different study populations ranged from a median 29 [5] to a mean age of $58^{12}$. Graded compression stockings at $30-40 \mathrm{~mm} \mathrm{Hg}$ for at least 6 months were prescribed in $36 \%$ of studies (5/14). Risk factors for DVT were presented in several studies; the prevalence of thrombophilia (e.g. factor $\mathrm{V}$ Leiden mutation, antithrombin III deficiency, protein $\mathrm{C}$ deficiency, antiphospholipid antibodies) ranged from $17 \%$ to $68 \%$ and the prevalence of cancer ranged from $4 \%{ }^{18}$ to $22 \%$.

\section{Studies}

Abu Rahma et al. in 2001, compared anticoagulation alone $(n=31)$ to CDT plus venoplasty/stenting $(n=18)$ in patients after acute iliofemoral DVT; ${ }^{4} 56 \%(10 / 18)$ of the latter group was stented. Three patients $(17 \%)$ in the intervention group had thrombophilia and $9 / 31(29 \%)$ in the comparator group; all patients with thrombophilia as well as all stented patients were on indefinite anticoagulant therapy. All other patients received warfarin for 6 months and in case of pulmonary embolism for 9-12 months.

Dayal et al. included 25 patients with acute and chronic critical thrombosis or occlusions at different anatomical sites in their study published in $2005 .{ }^{6}$ Participants underwent PMT, CDT, and lesions resistant to CDT were stented. Fifteen out of 25 patients (60\%) received stents. Lifelong anticoagulation was prescribed to $24 / 25$ patients. Data on thrombophilia were not presented. 
Venous stenting after deep venous thrombosis and antithrombotic therapy

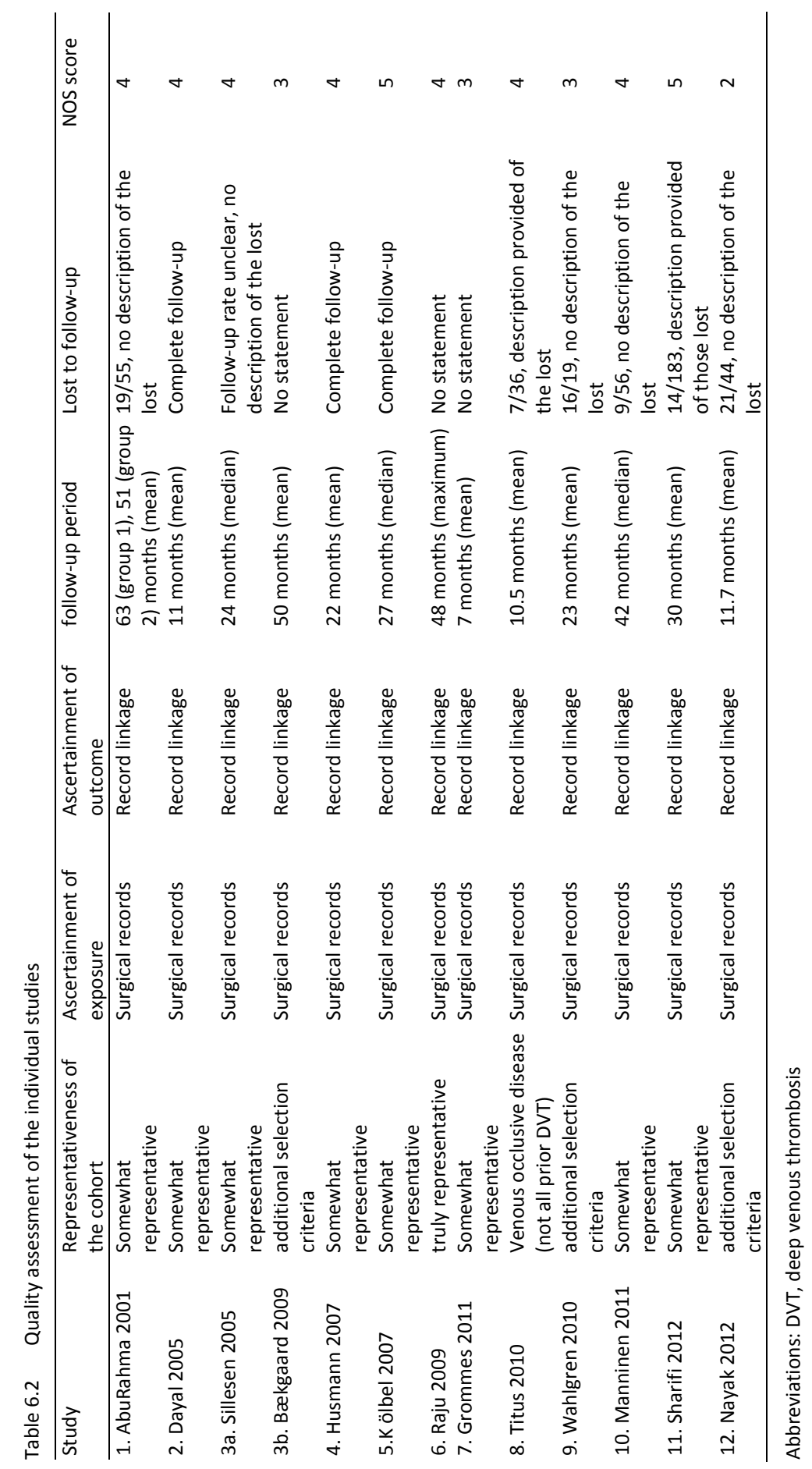


Sillesen et al. presented in 2005 their results of a retrospective analysis of outcomes of CDT in 45 patients with acute iliofemoral DVT. ${ }^{14}$ Treatment modalities consisted of CDT and, in case of the presence of residual thrombus, angioplasty plus stenting; thirty of 45 patients (67\%) were stented. Thrombophilia was diagnosed in 30/45 (67\%) of patients. All patients received warfarin for at least 12 months.

Bækgaard et al. 2009 reported on the same but extended population (101 patients, 103 limbs) as Sillesen et al, besides the earlier 45 patients 56 extra patients were included in three extra years of inclusion, all patients were followed prospectively. All patients underwent CDT and 57/103 (55\%) of limbs were stented. ${ }^{5}$ Anticoagulation treatment was given for at least 12 months to all participants; $54 \%$ of the population was diagnosed with thrombophilia.

In 2007 Husmann et al. published a retrospective analysis of 11 stented patients with acute DVT and May-Thurner disease. ${ }^{8}$ No data on thrombophilia were presented. Warfarin was installed for 6 months for all participants.

Kölbel et al. presented in 2007 results on 37 patients (44 limbs) with acute, extensive DVT of the iliocaval segment. ${ }^{9}$ Patients underwent CDT and in 36/44 limbs (82\%) stents for underlying stenosis or residual thrombosis were placed. Twenty-five out of 32 patients tested (78\%) for thrombophilia were tested positive for 1 or more hypercoagulable disorders. In patients with bilateral thrombosis this percentage was even higher $6 / 7$ (86\%). Warfarin therapy for 6 months was prescribed to all participants.

Raju et al, 2009. ${ }^{13}$ included during 9 years 167 limbs of 159 unselected patients with total occlusions of the iliac vein (acute and chronic DVT); all were stented. Of all patients, 44/159 (34\%) were diagnosed with 'significant thrombophilia' prior to the intervention. Long-term aspirin use was prescribed early in the study; patients with a known thrombophilia received additional warfarin. This practice was changed over time; Fondaparinux was used for 4-6 weeks in all and long-term anticoagulation was installed for selected patients (recanalizations $\geq 3$ segments, suprarenal stents, thrombophilia).

Grommes et al, 2011 studied a population of 12 patients (13 procedures), 11 patients with chronic or acute DVT in the lower extremities and 1 patient with superior caval vein thrombosis. ${ }^{7}$ All patients underwent CDT and 3/12 (25\%) were stented. Patients received anticoagulant therapy as advised in the ACCP guidelines 2008: 3 months for provoked DVT and 6 months for idiopathic DVT.

Oguzkurt et al. presented in 2009 a small case series of 7 patients with phlegmasia cerulea dolens secondary to acute iliofemoral DVT of which 3 had underlying MayThurner disease. ${ }^{12}$ CDT and stent placement procedures in $3 / 7(43 \%)$ were used as 
adjunctive procedures to endovascular treatment with manual thrombectomy. All patients received warfarin for 6 months.

In 2010 Titus et al. retrospectively reviewed the charts of a population consisting of 36 patients (40 limbs) with acute or chronic iliofemoral DVT or occlusions caused by external compression. ${ }^{17}$ Underlying thrombophilia was diagnosed in $22 \%$ of the patients checked. All patients underwent angioplasty and were stented; thrombolysis was performed in $52.8 \%$ of the cases. All patients received anticoagulation for 6 months (warfarin at INR 2-3 or Lovenox).

Wahlgren et al, 2010 conducted a study with a population of 50 patients (51 limbs) diagnosed with chronic PTS; 21 limbs (38\%) underwent endovascular and/or surgical treatment. ${ }^{18}$ All procedures consisted of angioplasty plus stenting. Thrombophilia testing of the total population resulted in 15/50 (30\%) positive results; in the stented population this was $10 / 20$ (50\%). Stented participants received oral anticoagulation for at least 6 months and aspirin for 1 month additionally.

Manninen et al. conducted in 2011 a study involving 56 patients with acute DVT extending to high femoral or iliac vein. ${ }^{10}$ All patients were treated with CDT; nine out of $56(16 \%)$ received 1 or more stents. Participants received warfarin for 6 months. Thrombophilia was established in $34 \%$ of the patients.

Sharifi et al. performed a randomized trial in 2012 comparing conservative anticoagulation therapy to percutaneous endovenous interventions plus anticoagulation (PEVI). The patient population of 183 patients consisted of patients with symptomatic proximal DVT. ${ }^{15}$ In the PEVI group $27 / 90(30 \%)$ of the patients were stented. All patients in the PEVI group received aspirin for a minimum of 6 months in addition to warfarin.

In 2012 Nayak et al. performed a study with a population of 44 patients (39 stented, 45 limbs) with PTS and acute or chronic DVT. Endovascular interventions were performed to treat established PTS and one or more stents were placed. In the subgroup of stented patients significant improvements after stent insertion were reported regarding the variables any pain and any swelling. Aspirin $81 \mathrm{mg} /$ day was administered to all participants; warfarin was only given when patients were already using warfarin prior to the intervention; no compression stockings were prescribed after the procedure. ${ }^{11}$

\section{Antithrombotic therapy}

Oral and/or parenteral anticoagulation was administered to all patients who underwent iliac venous stenting in $86 \%$ of the included studies $(12 / 14)^{4-10,12,14,15,17,18}$ only in two studies (2/14) anticoagulation was not installed in all participants. ${ }^{11,13}$ In 
the study performed by Nayak, only patients who were on warfarin prior to stent insertion received post-intervention anticoagulation; all participants, including those on warfarin received aspirin at daily doses of $81 \mathrm{mg} .{ }^{11}$ In the study performed by Raju, only patients with thrombophilia received warfarin. A not specified proportion of the population received only aspirin to maintain long-term stent patency. ${ }^{13}$ This practice was changed over time (not specified); later fondaparinux for 4 to 6 weeks was prescribed for all patients and long-term warfarin for selected patients (recanalizations $\geq 3$ segments, suprarenal stents, thrombophilia); no reason was provided for this change of practice and no differentiation was made between these regimes in terms of outcomes. ${ }^{13}$ Anticoagulant management in the included studies therefore can roughly be divided in 3 regimes: 1 ) Warfarin administration with a target International Normalized Ratio (INR) of 2-3 for 3 to 6 months concurrent with $\mathrm{LMWH}$ in the initial phase or in case of renal insufficiency UFH administration until the INR reaches a value of 2 for 2 consecutive days: $58 \%$ of studies $(8 / 12), 2$ ) Warfarin with an target INR of 2-3 for at least 12 months concurrent with LMWH or UFH in the initial phase: $17 \%$ of studies (2/12) and 3 ) warfarin administration, INR range 2-3 indefinitely concurrent with initial LMWH or UFH: $17 \%$ of studies (1/12). One remaining study could not be classified in one of these categories (8\%). These regimes were supplemented in $33 \%$ (4/12) of studies with 4) antiplatelet administration; aspirin or aspirin plus clopidogrel. For a detailed summary of antithrombotic therapy in the different studies see Table 6.3.

\section{Outcomes}

\section{Recurrent DVT and occlusions}

The incidence of recurrent DVT was reported in $12 / 14$ studies; ${ }^{4,5,7-10,12-15,17,18}$ The incidence of re-thrombosis in (sub) groups of only stented patients ranged in different studies from $5 \%{ }^{15}$ to $25 \%$ (early re-thrombosis $6 \%$; late re- thrombosis $19 \%$ ). ${ }^{13}$ In the study by AbuRahma et al. recurrent DVT occurred in 3/18 (17\%) of the patients in the endovenous intervention group and in $2 / 8(25 \%)$ in the comparator group. ${ }^{4}$ A fairly low rate of DVT was reported by Titus; $1 / 36$ (3\%), ${ }^{17}$ Sillesen; ${ }^{14} 1 / 45$ (2\%) and also in the study by Kölbel $1 / 44$ limbs (2\%). ${ }^{9}$ Bækgaard et al. reported 6/103 (6\%) recurrent DVT ( 3 early within days and 3 late within $2-3$ years). ${ }^{5}$ Four patients (31\%) experienced an early recurrent DVT in the study by Grommes et al. ${ }^{7}$ Recurrent DVT occurred in 2 patients $(29 \%)$ in the study by Oguzkurt et al. ${ }^{12}$ Post intervention DVT was diagnosed in $2 / 56$ (3.8\%) of patients by Manninen et al. ${ }^{10}$ After 6 months recurrent DVT was $2 \%$ in the PEVI group compared to $15 \%$ in the anticoagulation alone group $(p=0.003) .{ }^{15} \mathrm{In}$ - 
stent thrombosis was diagnosed in $1 / 11(9 \%)$ of the cases in the study by Husmann et al. $^{8}$ Early (<30 days) thrombosis of the stented iliac vein occurred in 3/21 stented limbs (14\%); no late re-thrombosis was recorded in the study by Wahlgren et al. ${ }^{18}$ Rethrombosis was seen in $42 / 167(25 \%)$ limbs by Raju et al.; early ( $<30$ days since stenting) in $10 \mathrm{limbs}$ and late in 32 limbs. ${ }^{13}$ Seven out of 43 patients (16\%) in the study by Nayak et al. experienced a stent occlusion during follow-up. ${ }^{11}$ Re-thrombosis or reocclusions occurred in 3/25 (12\%)in the study by Dayal et al.. ${ }^{6}$

Table 6.3 Suggested antithrombotic options

\begin{tabular}{|c|c|c|c|c|c|}
\hline Study & $\begin{array}{l}\text { Warfarin INR } \\
2-3 \text { for } 3 \text { to } 6 \\
\text { months }\end{array}$ & $\begin{array}{l}\text { Warfarin INR } \\
2-3 \text { for } 12 \\
\text { months }\end{array}$ & $\begin{array}{l}\text { Warfarin INR 2-3 } \\
\text { indefinitely }\end{array}$ & Antiplatelets & $\begin{array}{l}\text { Graded } \\
\text { compression } \\
\text { stockings }\end{array}$ \\
\hline $\begin{array}{l}\text { 1.AbuRahma } \\
2001\end{array}$ & $\begin{array}{l}\text { All remaining } \\
\text { patients }\end{array}$ & $\begin{array}{l}\text { Patients after } \\
\text { PE }\end{array}$ & $\begin{array}{l}\text { All stented, } \\
\text { patients with } \\
\text { thrombophilia }\end{array}$ & & \\
\hline 2.Dayal 2005 & & & $\mathrm{x}$ & & \\
\hline $\begin{array}{l}\text { 3a.Sillesen } \\
2005\end{array}$ & & $x$ & & & $x$ \\
\hline $\begin{array}{l}\text { 3b.Bækgaard } \\
2009\end{array}$ & & $x$ & & & $x$ \\
\hline $\begin{array}{l}\text { 4.Husmann } \\
2007\end{array}$ & $x$ & & & & \\
\hline 5.Kölbel 2007 & $\mathrm{x}$ & & & & $x$ \\
\hline $\begin{array}{l}\text { 6.Raju } 2009 \\
\text { earlier }\end{array}$ & $\begin{array}{l}\text { only in case o } \\
\text { warfarin }\end{array}$ & f thrombophilia & a: long term & Long term aspirin & \\
\hline $\begin{array}{l}\text { 6.Raju } 2009 \\
\text { later }\end{array}$ & $\begin{array}{l}\text { For most part } \\
\text { (4-6 weeks) ar } \\
\text { selected patie }\end{array}$ & $\begin{array}{l}\text { icipants: fondar } \\
\text { nd long term w } \\
\text { ents }\end{array}$ & $\begin{array}{l}\text { parinux } \\
\text { arfarin for }\end{array}$ & & \\
\hline 7.Grommes & X Idiopathic & & & & \\
\hline 2011 & $\begin{array}{l}\text { DVT: } 6 \\
\text { months, } \\
\text { provoked } \\
\text { DVT: } 3 \\
\text { months }\end{array}$ & & & & \\
\hline $\begin{array}{l}\text { 8.Oguzkurt } \\
2010\end{array}$ & $x$ & & & & \\
\hline 9.Titus 2010 & X or Lovenox & & & & \\
\hline $\begin{array}{l}\text { 10.Wahlgren } \\
2010\end{array}$ & $\mathrm{x}$ & & & $\begin{array}{l}\text { Aspirin } 1 \text { month (only for } \\
\text { patients after surgery) }\end{array}$ & \\
\hline $\begin{array}{l}\text { 11. Manninen } \\
2011\end{array}$ & $\mathrm{X}$ & & & & $x$ \\
\hline $\begin{array}{l}\text { 12.Sharifi } \\
2012\end{array}$ & $\begin{array}{l}\text { INR 2-3; no du } \\
\text { mentioned }\end{array}$ & uration anticoag & gulant use & $\begin{array}{l}\text { Aspirin at least } 6 \text { months+ } \\
\text { clopidogrel } 2 \text { to } 4 \text { weeks for } \\
\text { femoral/popliteal stents }\end{array}$ & $x$ \\
\hline $\begin{array}{l}\text { 13. Nayak } \\
2012\end{array}$ & $\begin{array}{l}\text { Only those wr } \\
\text { to interventio }\end{array}$ & ho were on anti & icoagulants prior & Aspirin for all participants & \\
\hline
\end{tabular}

Abbreviations: DVT, deep venous thrombosis; PE, pulmonary embolism 


\section{Patency rates}

Long-term stent patency rates were assessed in 10/15 studies; ${ }^{4-6,8-10,13,14,17,18}$ primary, assisted primary patency and secondary patency rates 12 months after stent placement ranged from $54 \%, 72 \%, 83 \%{ }^{13}$ respectively to $78 \%, 83 \%, 95 \%{ }^{17}$ in (sub)groups of only stented patients. High patency rates in a (sub) population of only stented patients were reported by Titus et al. After 12 months of follow-up primary, assisted primary and secondary patency rates were $78 \%, 83 \%$ and $95 \%$ respectively. These high patency rates can possibly be explained by the fact that also patients without a prior DVT were included in this study. ${ }^{17}$

\section{Pulmonary embolism}

In 11/14 studies the incidence of PE was reported. Three studies reported an incidence of $0 \%$ PE in (sub) groups of only stented patients. ${ }^{11,13,17}$ Nayak et al. reported PE incidence of a subgroup of stented patients with PTS; the prevalence of inferior vena cava (IVC) filters was not reported, ${ }^{11}$ Raju et al. reported zero PE in a population of stented patients with post-thrombotic chronic total occlusions; the prevalence of IVC filters was $10 \%{ }^{13}$ and Titus et al. studied patients undergoing iliofemoral venous angioplasty and stenting; IVC filters were placed in case of very high PE risk. ${ }^{17}$ The population with the highest incidence of PE (7\%), studied by Manninen et al. consisted of patients with acute iliofemoral DVT; in $9 \%$ of the patients an IVC filter was placed during the procedure. ${ }^{10}$ In the studies, performed by Abu Rahma (in endovascular intervention group), Grommes, Kölbel, and Oguzkurt 0\% PE was reported in partially stented populations. ${ }^{4,7,9,12}$ The remaining studies by Bækgaard, Sillesen, and Sharifi (in endovascular intervention group) reported $6 \%, 2 \%$ and $1 \%$ of PE respectively. ${ }^{5,14,15}$

\section{Post thrombotic syndrome}

PTS was assessed in 6/14 studies. ${ }^{8,11,13,15,17,18}$; in only 2 studies ${ }^{8,18}$ the Villalta-Prandoni scale or the Venous Clinical Severity Score was used, both validated in different settings ${ }^{24-26}$ and subsequently endorsed by the International Society on Thrombosis and Hemostasis (ISTH).

One of these studies, a study performed by Husmann et al. assessed PTS in a population of only venous stented participants with May-Thurner syndrome and DVT: 10/11 stented patients with a target INR of 2-3 for the first 6 months had a Villalta score of 0 after a mean follow-up of 22 months. Duplex ultrasonography revealed no post-thrombotic changes in these patients; 1 patient reported mild PTS symptoms. Patients did not receive compression stockings after the intervention. ${ }^{8}$ 
Post-hoc analysis performed after a trial by Sharifi et al. suggested a protective effect of aspirin, on top of warfarin treatment (INR 2-3) in reducing the prevalence of PTS; relative risk (RR) of 0.37 , confidence interval $(\mathrm{Cl}) 0.19$ to 0.74 . In the endovascular intervention plus anticoagulation group $7 \%$ PTS was reported versus $30 \%$ in the anticoagulation alone group $(p<0.001)$. All patients wear advised to wear compression stockings; however, 6 months compliance was only $27 \%{ }^{15}$

A Chronic Venous Insufficiency Questionnaire was completed before and after the recanalization of 128 limbs in the report presented by Raju; significant improvements were noted concerning leg pain, sleep disturbance, effect on social activities and morale after the intervention. The hemodynamic parameters hand-foot pressure and ambulatory venous pressure showed no significant improvements. No graded compression stockings were prescribed post procedurally. ${ }^{13}$

Nayak et al. reported significant improvements after stent insertion for the variables any pain and any swelling as proxy for PTS; no compression stockings were prescribed after the procedure. ${ }^{11}$ Titus et al assessed by telephone whether symptoms overall had improved, had not changed, or became progressively worse: a distribution of $83 \%, 7 \%$ and $10 \%$ was found. In this study patients did not receive compression stockings. $^{17}$

Finally, Wahlgren et al. reported Venous Clinical Severity scores before and after the intervention; the score dropped from 9.1 points preprocedural to 6.0 points postprocedural $(p<0.0001)$. Patients were not advised to wear stockings post procedurally. ${ }^{18}$

\section{Major bleeding}

Finally we explored rates of major bleeding. Five studies reported $0 \%$ major bleedings during long term follow-up. ${ }^{11-14,17}$ In only 1 of these studies, performed by Nayak et al. all patients received aspirin, and anticoagulation was installed for those prior on warfarin; in the subgroup of stented patients no major bleeding occurred. ${ }^{11}$

In another study by Raju et al. in which no major bleeding complications were reported only a part of the population received aspirin plus anticoagulation; the remaining part received anticoagulation or aspirin. ${ }^{13}$

In the remaining studies with $0 \%$ major bleeding complications all patients received warfarin for different time periods. The highest major bleeding scores (11\%) were reported in a population, described by Abu Rahma et al, consisting of patients with iliofemoral DVT, of which $56 \%$ of the participants were stented. All patients were on warfarin (INR 2-3) indefinitely, without the addition of aspirin. ${ }^{4}$ Studies performed by 
Bækgaard, Grommes, Kölbel and Manninen reported major bleeding rates of $1 \%, 8 \%$, $8 \%$ and $4 \%$ respectively. ${ }^{5,7,9,10}$ In all these studies patients received only warfarin.

\section{Discussion}

Venous stenting is performed in case residual thrombi remain after $\mathrm{CDT}^{9,14}$ or lesions appear to be resistant to angioplasty. ${ }^{6}$ Overall, the evidence supporting the use of venous stents after a DVT in the iliofemoral area for improved short-term and longterm patency is convincing although of limited methodological quality. The lack of comparator studies is evident: only 2 studies $^{4,15}$ of which 1 trial $^{15}$ compared venous surgery plus anticoagulation to anticoagulation alone. In these studies the venous surgery group (not all stented) performed significantly better than the anticoagulation alone group in terms of patency, ${ }^{4}$ recurrent venous thromboembolism and development of PTS. ${ }^{15}$ Interestingly, in many cases thrombophilia screening is performed, but only on limited occasions type and duration of antithrombotic treatment are based on the outcomes of its findings. ${ }^{4,13}$ Post procedural antithrombotic therapy is not assessed separately as a potential factor for short-term and long-term failure or success of the stenting procedure in any of the presented studies. Based on this review we conclude that most stented patients receive warfarin only for the standard duration of the treatment of their underlying DVT; the fact that venous stents are inserted does not seem to affect this antithrombotic regime. This practice is in accordance with current ACCP guidelines. ${ }^{21}$ In only 4 studies patients received additional antiplatelet therapy after CDT and/or PMT and stent placement. ${ }^{11,13,16,18}$ In 1 study clopidogrel at $75 \mathrm{mg}$ per day for 2-4 weeks was administered to exclusively stented patients. ${ }^{16}$ Combination therapy, consisting of anticoagulant and antiplatelet therapy or dual antiplatelet therapies (aspirin plus clopidogrel) may theoretically be an interesting option. It is conceivable that antiplatelet agents could provide additional protection against the formation of thrombi after venous stenting of the iliac vein. ${ }^{27}$ Shear forces are a major determinant of platelet deposition in thrombi; in the venous system usually low-flow, low-shear conditions exist and occurring thrombi are likely platelet poor and fibrin rich. In the iliac vein however, due to thickening and scarification of the vessel wall after a DVT and/or external compression, shear forces are in some cases anticipated to be nearly identical to the arterial system. ${ }^{27}$ In experimentally induced thrombi in these venous segments, indeed platelet rich thrombi occurred and these platelets were in an activated state. ${ }^{27}$ However, aspirin, clopidogrel, and aspirin plus clopidogrel did not 
significantly reduce stent thrombosis in an experimental model neither did they prevent platelet accumulation, while anticoagulant therapy did reduce thrombosis. ${ }^{27}$ The lowest patency rates were reported by Wahlgren, Dayal and Raju: ${ }^{6,13,18}$ Low patency was combined with high rates of recurrent DVT $(14 \%, 12 \%$ and $25 \%$ respectively). In these studies acute and chronic DVT, and in the case of Dayal also DVT at unusual sites were included. As far as the antithrombotic regimen that was applied in these studies is concerned, no beneficial effect was observed by the additional aspirin administration in those stented. ${ }^{13,18}$ The low patency rates in these studies can at least partially be explained by the fact that patients with chronic DVT and with total occlusions were included. In the study by Nayak aspirin was prescribed to all, and additional warfarin to those with previous use of warfarin; the recurrence rate was $16 \%{ }^{11}$ Besides the antithrombotic effects also anti-inflammatory effects could play a role while using antiplatelet agents. There could be an additional effect of aspirin on top of warfarin treatment for the reduction of the prevalence of PTS, as was suggested by the post-hoc analysis performed by Sharifi et al. ${ }^{15}$ that showed a significant reduction of the incidence of PTS in patients on aspirin.

The safety of antiplatelet therapy in combination with anticoagulation in patients after venous stenting is something to be considered. Limited data are provided by 2 studies by Nayak and Raju, wherein aspirin plus warfarin therapy was applied and $0 \%$ major bleedings were reported, ${ }^{11,13}$ the remaining 2 studies in which the patients received antiplatelet plus anticoagulant therapy also no major bleedings were reported. ${ }^{15,18}$ Therefore in terms of safety, the addition of aspirin to the antithrombotic regimen does not seem to pose a large problem, at least on the short term in the patients selected in these studies.

The highest primary patency rates were reported in studies performed by Manninen, Sillesen and Abu Rahma; ${ }^{4,10,14}$ all patients received warfarin with a target INR 2-3 for the standard duration for the underlying DVT, and no antiplatelet therapy was administered. In all three studies only patients with acute DVT were included; fresh thrombi probably resolve better after CDT than sub acute thrombi. ${ }^{28}$ Also low rates of recurrent thrombosis were reported in these studies. These 3 studies were all awarded with $4 / 5$ stars for study quality; we therefore can assume that the study findings are trustworthy. Kölbel also reported low rates of recurrent DVT and PE. ${ }^{9} \mathrm{~A}$ very high percentage $(68 \%)$ of the total study population was diagnosed with thrombophilia; this did however not influence antithrombotic management.

Anticoagulants likely play a more important role than antiplatelet agents in the prevention of re-stenosis in venous stented patients with a prior DVT. ${ }^{2,21}$ Recurrent venous thrombosis is primarily caused by increased thrombin generation, ${ }^{29}$ therefore anticoagulants likely have better antithrombotic properties in patients with venous 
stents after a DVT than antiplatelet drugs. The major bleeding risk of long-term warfarin use with a target INR of 2-3 was estimated at $1.3 \%$ per year. ${ }^{30}$ The rates of major bleeding reported in this review are in a higher range between $8-11 \%$. The additional bleeding risk is mainly due to the stenting procedure. This may be an acceptable risk if stenting would lead to clearly better outcomes.

Based on the available evidence in this review no firm conclusions regarding the association between the duration of antithrombotic therapy and the study outcomes can be drawn. A major limitation of this study is the fact that no meta-analysis could be performed. The quality of the included studies was sub optimal; single armed cohort studies with mostly a small sample size formed the majority in this review. Applicability of the review may be affected by the fact that most studies assessed patient groups undergoing CDT and/or PMT of which only a part underwent stenting; subgroups consisting of only stented patients could be assessed in only a few cases. The most important factors contributing to a favorable outcome, high patency and low recurrence rate are mainly dependent on the location of the thrombus and the age of the thrombus. Thrombophilia is not a discriminating factor for success. Anticoagulant therapy for the duration of the underlying thrombotic lesions seems to be sufficient.

The conclusion of this systematic literature review is that antithrombotic therapy does not seem to influence any of the outcomes in patients with venous stenting after DVT: patency, PTS, PE, recurrent DVT or restenosis and bleeding. The main drivers for the outcome are the location of the DVT, the age of the thrombus and the stenting procedure itself. 


\section{References}

1. Jenkins JS. Endovascular therapies to treat iliofemoral deep venous thrombosis. Prog Cardiovasc Dis. 2011;54:70-6.

2. Meissner MH. Indications for platelet aggregation inhibitors after venous stents. Phlebology. 2013;28 Suppl 1:91-8.

3. Mackman N. Triggers, targets and treatments for thrombosis. Nature. 2008;451:914-8.

4. AbuRahma AF, Perkins SE, Wulu JT, Ng HK. Iliofemoral deep vein thrombosis: conventional therapy versus lysis and percutaneous transluminal angioplasty and stenting. Ann Surg. 2001;233:752-60.

5. Baekgaard N, Broholm R, Just S, Jorgensen M, Jensen LP. Long-term results using catheter-directed thrombolysis in 103 lower limbs with acute iliofemoral venous thrombosis. Eur J Vasc Endovasc Surg. 2010;39:112-7.

6. Dayal R, Bernheim J, Clair DG, Mousa AY, Hollenbeck S, DeRubertis B, et al. Multimodal percutaneous intervention for critical venous occlusive disease. Ann Vasc Surg. 2005;19:235-40.

7. Grommes J, Strijkers R, Greiner A, Mahnken AH, Wittens CH. Safety and feasibility of ultrasoundaccelerated catheter-directed thrombolysis in deep vein thrombosis. Eur J Vasc Endovasc Surg. 2011;41:526-32.

8. Husmann MJ, Heller G, Kalka C, Savolainen H, Do DD, Schmidli J, et al. Stenting of common iliac vein obstructions combined with regional thrombolysis and thrombectomy in acute deep vein thrombosis. Eur J Vasc Endovasc Surg. 2007;34:87-91.

9. Kolbel T, Lindh M, Holst J, Uher P, Eriksson KF, Sonesson B, et al. Extensive acute deep vein thrombosis of the iliocaval segment: midterm results of thrombolysis and stent placement. J Vasc Interv Radiol. 2007;18:243-50.

10. Manninen $\mathrm{H}$, Juutilainen A, Kaukanen $\mathrm{E}$, Lehto $\mathrm{S}$. Catheter-directed thrombolysis of proximal lower extremity deep vein thrombosis: a prospective trial with venographic and clinical follow-up. Eur J Radiol. 2012;81:1197-202.

11. Nayak L, Hildebolt CF, Vedantham S. Postthrombotic syndrome: feasibility of a strategy of imagingguided endovascular intervention. J Vasc Interv Radiol. 2012;23:1165-73.

12. Oguzkurt L, Ozkan U, Demirturk OS, Gur S. Endovascular treatment of phlegmasia cerulea dolens with impending venous gangrene: manual aspiration thrombectomy as the first-line thrombus removal method. Cardiovasc Intervent Radiol. 2011;34:1214-21.

13. Raju S, Neglen P. Percutaneous recanalization of total occlusions of the iliac vein. J Vasc Surg. 2009;50:360-8.

14. Sillesen $H$, Just $S$, Jorgensen $M$, Baekgaard N. Catheter directed thrombolysis for treatment of iliofemoral deep venous thrombosis is durable, preserves venous valve function and may prevent chronic venous insufficiency. Eur J Vasc Endovasc Surg. 2005;30:556-62.

15. Sharifi M, Bay C, Mehdipour M, Sharifi J. Thrombus Obliteration by Rapid Percutaneous Endovenous Intervention in Deep Venous Occlusion (TORPEDO) trial: midterm results. J Endovasc Ther. 2012;19:273-80.

16. Sharifi M, Mehdipour M, Bay C, Smith G, Sharifi J. Endovenous therapy for deep venous thrombosis: the TORPEDO trial. Catheter Cardiovasc Interv. 2010;76:316-25.

17. Titus JM, Moise MA, Bena J, Lyden SP, Clair DG. Iliofemoral stenting for venous occlusive disease. J Vasc Surg. 2011;53:706-12.

18. Wahlgren $\mathrm{CM}$, Wahlberg E, Olofsson P. Endovascular treatment in postthrombotic syndrome. Vasc Endovascular Surg. 2010;44:356-60.

19. Neglen P, Hollis KC, Olivier J, Raju S. Stenting of the venous outflow in chronic venous disease: longterm stent-related outcome, clinical, and hemodynamic result. J Vasc Surg. 2007;46:979-90.

20. Ye K, Lu X, Li W, Huang Y, Huang X, Lu M, et al. Long-term outcomes of stent placement for symptomatic nonthrombotic iliac vein compression lesions in chronic venous disease. J Vasc Interv Radiol. 2012;23:497-502. 


\section{Chapter 6}

21. Kearon C, Akl EA, Comerota AJ, Prandoni P, Bounameaux H, Goldhaber SZ, et al. Antithrombotic therapy for VTE disease: Antithrombotic Therapy and Prevention of Thrombosis, 9th ed: American College of Chest Physicians Evidence-Based Clinical Practice Guidelines. Chest. 2012;141(2 Suppl):e419S-94S.

22. Sanderson S, Tatt ID, Higgins JP. Tools for assessing quality and susceptibility to bias in observational studies in epidemiology: a systematic review and annotated bibliography. Int J Epidemiol. 2007;36:666-76.

23. Stang A. Critical evaluation of the Newcastle-Ottawa scale for the assessment of the quality of nonrandomized studies in meta-analyses. Eur J Epidemiol. 2010;25:603-5.

24. Jayaraj A, Meissner MH. A comparison of villalta-prandoni scale and venous clinical severity score in the assessment of post thrombotic syndrome. Ann Vasc Surg 2014;28:313-7.

25. Galanaud JP, Holcroft CA, Rodger MA, Kovacs MJ, Betancourt MT, Wells PS, et al. Comparison of the Villalta post-thrombotic syndrome score in the ipsilateral vs. contralateral leg after a first unprovoked deep vein thrombosis. J Thromb Haemost. 2012;10:1036-42.

26. Kahn SR. Measurement properties of the Villalta scale to define and classify the severity of the postthrombotic syndrome. J Thromb Haemost. 2009;7:884-8.

27. McBane RD, 2nd, Leadley RJ, Jr., Baxi SM, Karnicki K, Wysokinski W. Iliac venous stenting antithrombotic efficacy of PD0348292, an oral direct Factor Xa inhibitor, compared with antiplatelet agents in pigs. Arterioscler Thromb Vasc Biol. 2008;28:413-8.

28. Zhang X, Ren $Q$, Jiang X, Sun J, Gong J, Tang B, et al. A prospective randomized trial of catheterdirected thrombolysis with additional balloon dilatation for iliofemoral deep venous thrombosis: a single-center experience. Cardiovasc Intervent Radiol. 2014;37:958-68.

29. Geerts WH, Pineo GF, Heit JA, Bergqvist D, Lassen MR, Colwell CW, et al. Prevention of venous thromboembolism: the Seventh ACCP Conference on Antithrombotic and Thrombolytic Therapy. Chest. 2004;126(3 Suppl):338S-400S.

30. Levine MN, Raskob G, Landefeld S, Kearon C. Hemorrhagic complications of anticoagulant treatment. Chest. 2001;119(1 Suppl):108-21. 


\section{Appendix 6.1 The search strategy for MEDLINE}

((stents[MeSH Terms] OR stent[MeSH Terms] OR angioplasty[MeSH Terms] OR Percutaneous recanalization OR endovascular interventions) AND (venous ulcer[MeSH Terms] OR venous ulcers[MeSH Terms] OR venous insufficiencies[MeSH Terms] OR venous insufficiency[MeSH Terms] OR "postthrombotic syndrome"[MeSH Major Topic] OR deep venous thromboses[MeSH Terms] OR deep venous thrombosis[MeSH Terms]) AND (agents, anticoagulant[MeSH Terms] OR anticoagulant agents[MeSH Terms] OR anticoagulant drugs[MeSH Terms] OR anticoagulants[MeSH Terms] OR drugs, anticoagulant[MeSH Terms] OR blood platelet aggregation inhibitors[MeSH Terms] OR agents, antiplatelet[MeSH Terms] OR antiplatelet agents[MeSH Terms] OR antiplatelet drugs[MeSH Terms] OR drugs, antiplatelet[MeSH Terms])) OR ((stent OR angioplasty OR Percutaneous recanalization) AND (venous ulcer[MeSH Terms] OR venous ulcers[MeSH Terms] OR venous insufficiencies[MeSH Terms] OR venous insufficiency[MeSH Terms] OR "postthrombotic syndrome"[MeSH Major Topic] OR deep venous thromboses[MeSH Terms] OR deep venous thrombosis[MeSH Terms]) $A N D$ (anticoagulant $O R$ anticoagulant agents $O R$ anticoagulant drugs $O R$ anticoagulants $O R$ blood platelet aggregation inhibitors $O R$ Antiplatelet $O R$ antiplatelet agents OR antiplatelet drugs))

Limits Activated MEDLINE: English 


\section{Appendix 6.2 Newcastle-Ottawa quality assessment scale for cohort studies (selection of 5 items}

Selection

1) Representativeness of the cohort
a. truly representative of the population of patients undergoing venous stenting in the iliofemoral area after a first or recurrent DVT; all participants stented *
b. somewhat representative of the population of patients undergoing venous stenting in the iliofemoral area after a first or recurrent DVT; not all participants stented *
c. additional inclusion criteria and not all participants stented.
d. no description of the derivation of the cohort

2) Ascertainment of exposure
a. secure record (e.g. surgical records) *
b. structured interview *
c. written self report
d. no description

Outcome

3) Assessment of outcome
a. independent blind assessment *
b. record linkage *
c. self report
d. no description

4) Was follow-up long enough for outcomes to occur
a. yes ( $\geq 24$ months (mean or median)) *
b. no

5) Adequacy of follow-up of cohorts
a. complete follow-up - all subjects accounted for *
b. subjects lost to follow-up $(<5 \%)$ unlikely to introduce bias or description provided of those lost *
c. follow-up rate $<95 \%$ and no description of those lost
d. no statement 


\section{Chapter 7}

\section{Quality of anticoagulant therapy and in-stent thrombosis in patients with venous stents}

Pieter Eijgenraam, Mark A.F. de Wolf, Ralph L.M. Kurstjens, Hugo ten Cate, Cees H.A. Wittens, Arina J ten Cate-Hoek

Submitted 


\begin{abstract}
Introduction

In patients with venous outflow obstruction following lliofemoral deep vein thrombosis (IFDVT) stenting of venous tracts to prevent or alleviate post thrombotic syndrome (PTS) is applied with increasing frequency. Up till now the association of quality of anti-thrombotic therapy and effectiveness of stenting has not been studied explicitly. The impact of the quality of anticoagulant therapy with vitamin $\mathrm{K}$ antagonists (VKA) expressed as time within therapeutic range (TTR) and proportion of INR values $<2.0$ on in-stent thrombosis is currently unknown.
\end{abstract}

\title{
Methods
}

Seventy-nine patients with venous stent placement after acute or chronic iliofemoral or caval DVT were included in this study. All patients used VKA after the intervention. TTR was calculated according to the linear interpolation method. In-stent thrombosis was assessed by the use of duplex ultrasound. Survival analysis (Cox regression, Kaplan Meier curves) was used to analyze the data.

\section{Results}

The average TTR was $46.4 \%$ (SD 20.8); the average proportion of INR measurements $<2.0$ was 9.5\% (SD 11.6). Increasing TTR was associated with a lower risk of in-stent-thrombosis. The multivariable adjusted analysis resulted in a hazard ratio of $0.940(95 \% \mathrm{Cl} 0.890-0.993)$ per $1 \%$ increase in TTR. An increase of the proportion of INR values <2.0 was not significantly associated with the outcome in-stent thrombosis; HR per $1 \%$ increase in proportion of 1.018 (95\% Cl 0.957-1.084).

\section{Conclusions}

We conclude that an increase of TTR offers protection against in-stent thrombosis. The proportion of INR $<2.0$, although expected to be an important predictor for in-stent thrombosis, could not be identified as a risk factor in this study. An overall low average TTR of $46.4 \%$ (SD 20.8) was recorded, possibly due to the relative short follow-up in combination with the initiation phase of VKA, leading to unstable INR values. 


\section{Introduction}

Post thrombotic syndrome (PTS) develops in over $50 \%$ of patients with iliofemoral deep venous thrombosis (IFDVT). ${ }^{1}$ Conservative treatment regimens comprising of a combination of compression therapy, mobilization and oral anticoagulants, up till now mainly vitamin $\mathrm{K}$ antagonists (VKA), result in suboptimal outcomes in these patients. Over the last 2 decades the use of percutaneous transluminal angioplasty (PTA) and stenting of venous outflow obstructions of the iliofemoral and caval veins has gained more attention. PTA and stenting appears to be effective in terms of objective and subjective clinical improvement of PTS symptoms and has been characterized by good mid- to long-term patency rates in mainly observational studies. ${ }^{2-8}$ The treatment usually consists of guide-wire recanalization of the obstructed venous segments and placement of one or more stents, followed by anticoagulant therapy with VKA for at least 3 months. In acute DVT patients in whom catheter directed thrombolysis (CDT) is applied, additional venous stenting immediately following thrombolysis is often indicated in case of underlying venous pathology, such as iliac vein compression syndromes, which is thought to be the underlying cause of thrombosis in these patients. $^{9}$

So far the contribution of the quality of anticoagulant therapy, to the effectiveness of venous stenting has not been evaluated. ${ }^{10}$ The current guidelines issued by the American College of Chest Physicians (ACCP) do not specifically advise on anticoagulation therapy after venous stenting mainly for lack of evidence. ${ }^{11}$ In the literature a variety of antithrombotic therapies are suggested after venous stent placement for chronic venous obstruction or directly after CDT in acute thrombosis; anticoagulant therapy varying from 3 months to 1 year with target range INR of usually 2 to 3 is prescribed, sometimes in combination with antiplatelet therapy. ${ }^{10}$ While time within therapeutic range (TTR) of VKA therapy in acute DVT is known to have substantial impact on recurrent DVT, ${ }^{12,13}$ the role of the quality of TTR during VKA therapy in preventing re-occlusion after venous stenting remains unclear. In addition INR values $<2.0$ are an established risk factor for recurrent venous thromboembolism (VTE), ${ }^{13}$ as well as for PTS. ${ }^{14,15}$ Even though the contribution of the quality of anticoagulation therapy will therefore likely also play a role in the effectiveness of venous stenting, so far no study has analyzed TTR and proportion of INR values $<2.0$ in patients undergoing venous stenting in acute DVT or for chronic venous obstruction in relation to stent patency.

We therefore evaluated the contribution of the quality of anticoagulation control during treatment with VKA calculated with the linear interpolation method. In addition we assessed the impact of the proportion of INR values $<2.0$ on in-stent 
thrombosis in chronic PTS patients on long-term anticoagulation with iliofemoral and or caval venous stents placed in patients with chronic PTS.

\section{Methods}

\section{Patient selection}

During the period from March 2009 until January 2013108 patients, referred to the Department of Vascular Surgery in the Maastricht University Medical Center, the Netherlands for venous stent placement after acute or chronic iliofemoral or caval DVT, and who had used VKA for at least 3 months after the intervention and had at least 1 year of follow up data available were invited to participate in the current study. Patients with symptomatic deep venous obstruction without a history of DVT; i.e. cases of iliac vein compression syndrome (such as May-Thurner syndrome) were not eligible. Seventy-nine patients of whom $82.4 \%$ used VKA prior to the intervention, gave informed consent to retrieve patient data from their anticoagulation clinic records. All data, except data concerning anticoagulation therapy were collected prospectively. Institutional review board approval was obtained (METC 14-4-081).

\section{Baseline work-up}

All patients were scheduled for a standardized work-op, consisting of clinical evaluation, duplex ultrasound (DUS) and magnetic resonance venography (MRV) prior to the intervention. The primary objective using DUS is the assessment of signs of recanalization and/or flow impairment, luminal narrowing, vein wall thickening and any possible external compression on vein segments, such as iliac vein compression syndrome. During follow-up DUS was used to assess patency of stented venous tracts and vein segments proximal and distal of the stents, as well as the configuration of the stents themselves. The LET score, a classification that stratifies patients into 4 groups (I-IV) in accordance to the location of the most proximal thrombus, was used to classify patients. ${ }^{16}$ With MRV the extend and severity of intraluminal post thrombotic changes and collateralization, and the locations of trabeculae, longitudinal vertical strands attached to the vein wall that indicate PTS, were recorded. ${ }^{17}$

\section{Stent placement}

All procedures were performed under general anesthesia in a dedicated angiosuite. In patients using VKA at the time of intervention, the procedure was performed under full anticoagulation; i.e. coumadins were not stopped in the days preceding the 
stenting procedure if INR was $<4$. In patients not using anticoagulation at the time of intervention, $5.000 \mathrm{IU}$ of unfractionated heparin was administered at the start of the procedure, and another $5.000 \mathrm{IU}$ was given at the discretion of the treating physician in case of prolonged duration of the intervention. Venous access was obtained via the femoral vein under ultrasound guidance, generally at least $15 \mathrm{~cm}$ distally to the deep femoral inflow. With the use of various guide wires recanalization into the vena cava inferior was performed. ${ }^{18}$ As only stented patients were included in this study, the success rate for recanalization was set at $100 \%$. Following recanalization predilatation, i.e. PTA, of the obstructed vein segments was performed in the iliofemoral tract. Following pre-dilatation the affected vein segments were stented. The proximal and distal landing zones of the stents were placed in "healthy vein". Self-expendable stents, ranging in size of $12-16 \mathrm{~mm}$ in the iliofemoral tract, and $25 \mathrm{~mm}$ in the caval tract, were used in all cases (sinus $\mathrm{XL}$, sinus Venous and sinus $\mathrm{XL}$-flex, Optimed, Ettlingen, Germany and Zilver Vena (Cook, Galway, Ireland). For a small number of bilaterally affected patients balloon expendable stents were used to reconstruct the confluens of the vena cava ((AndraStent, Andramed, Reutlingen, Germany). ${ }^{19}$

In patients using VKA prior to the intervention, anticoagulant therapy was continued for at least 6 months following the intervention. All other patients received LMWH directly after the procedure, and were started on VKA on the first day postintervention. Stenting procedures were performed for three different indications: CDT + stenting after acute DVT, stenting after (chronic) PTS, and stenting with endophlebectomy and AV fistula after (chronic) PTS.

\section{Follow up and outcomes}

Patients were assessed at 2 weeks, 6 weeks, and 3, 6 and 12 months after the intervention and annually thereafter, or earlier when necessary, for a regular checkup. A standardized clinical assessment by a trained physician was performed and DUS to assess patency and possible stent configuration problems (kinking, migration, fracture, changes in orientation). In-stent thrombosis was defined as thrombosis with $100 \%$ occlusion of the treated vein. An endpoint for the study was reached when VKA administration was stopped, or when the last patency measurement was performed or when in-stent thrombosis occurred.

\section{Anticoagulation}

Patient records were retrieved from patient's individual anticoagulation clinics. Patient records included INR values, start and stop dates of VKA, indications for VKA therapy, target INR values, type of VKA (acenocoumarol, fenprocoumon) and 
instructions on the need to administer low molecular weight heparin (LMWH) in case of INR $<2.5$. The time within the therapeutic range (TTR) was calculated for all participants using the linear interpolation method as proposed by Rosendaal et al. ${ }^{20}$ This method assumes a linear relationship in time between 2 INR measurements. The first INR taken into account after the start of VKA treatment had a value of 2.0 or higher and was preceded by another INR measurement of 2.0 or higher. Hereafter all INR values were included for TTR calculation until the time the outcome occurred or the last patency measurement was performed. Furthermore the proportion of INR measurements with a value below 2.0 was calculated for each patient. In our analysis we also included the variables TTR and proportion of INR values $<2.0$ restricted to the first 8 weeks post-surgery to investigate the effect of the quality of anticoagulation of the (re)initiation phase on in-stent thrombosis. Some patients switched during the follow up from VKA to rivaroxaban or antiplatelet therapy; this was recorded as well.

\section{Statistical analysis}

Descriptive statistics were used to determine baseline characteristics. Continuous variables are reported as means, their standard deviations (SD); categorical data are presented as percentages. Student's T-tests and $\mathrm{Chi}^{2}$ tests were performed to compare groups. Kaplan Meier curves are presented for the comparison of the outcome in-stent thrombosis between the group with a TTR $\leq$ the median value of the group without in-stent thrombosis (control group) and a TTR $>$ than median control group. To compare groups log rank tests were performed. The Cox regression was used to express the association between the independent variables TTR and percentage of INR measurements $<2.0$ separately, corrected for possible confounders in relation to the outcome in-stent thrombosis. Interactions between TTR, proportion of INR values $<2.0$ and INR target range (2.5-3.5 and 3.0-4.0) were assessed. Person time was calculated from the time of the intervention up to the time when the outcome of interest occurred, the patient stopped using VKA or to the time of the last patency assessment was performed. Risks are presented as hazard ratios (HR) with accompanying confidence intervals $(\mathrm{Cl})$ or $\mathrm{p}$ - value. We used two ways to determine which variables finally entered the multivariable-adjusted models: 1 ) the potential confounder is predefined, i.e. in literature the association between the exposure of interest and in stent-thrombosis was described; or 2) the potential confounder is associated with in-stent thrombosis ( $p$-value $<0.20$ ). We selected the variables age at inclusion, gender, LET score, LMWH administration if INR <2.5 (yes/no) and VKA type (acenocoumarol, fenprocoumon). The proportional hazards assumption was tested for all variables. The analyses were restricted to patients with chronic iliofemoral DVT; we 
therefore excluded 9 patients with acute DVT + compression syndrome, because these patients have a different pathology and the effect of TTR on in-stent thrombosis therefore probably is different. SPSS version 22 was used for all analyses.

\section{Results}

Patient characteristics are detailed in Table 7.1. The average age at baseline was 41.5 years (SD 14.3 years) and $34.2 \%$ were male. We found an average TTR of $46.4 \%$ (SD 20.8) for the whole group. In the total population $20.3 \%$ of patients developed instent thrombosis; $15.0 \%$ were diagnosed with in-stent thrombosis in the group of PTS patients with an INR target range of $2.5-3.5$, and $25.0 \%$ acquired in-stent thrombosis in the group of patients with range $3-4,(p=0.27)$.

Table 7.1 Patient characteristics

\begin{tabular}{lll}
\hline age & 79 & 41.5 (SD 14.3) \\
Sex & 79 & $34.2 \%$ \\
Male & 27 & $65.8 \%$ \\
Female & 52 & \\
Thrombophilia & & $67.1 \%$ \\
$\quad$ No test performed & 53 & $16.5 \%$ \\
$\quad$ No thrombofilia & 13 & $13.9 \%$ \\
Factor V Leiden & 11 & $1.3 \%$ \\
Antiphopholipid syndrome & 1 & $1.3 \%$ \\
Factor V Leiden + prothrombin G20210A mutation & 1 & \\
Underlying pathology & & $7.6 \%$ \\
Acute DVT with compression syndrome & $62.4 \%$ \\
Post thrombotic syndrome & 73 & \\
Most proximal segment LET score according to DUS & & $67.1 \%$ \\
LET III & 53 & $16.5 \%$ \\
LET IV & 13 & $16.5 \%$ \\
No LET score available & 13 & \\
Outcomes & & \\
In stent thrombosis & & $79.7 \%$ \\
No & & $20.3 \%$ \\
Yes & 63 &
\end{tabular}

Abbreviations: DUS, duplex ultrasonography; DVT, deep venous thrombosis; LET, lower extremity thrombosis

TTR was higher for patients with INR values included during a period longer than 408 days (median follow up) compared to those with a shorter period of INR values available; $56.7 \%$ and $33.3 \%$ respectively $(p<0.001)$. For all anticoagulation characteristics: see Table 7.2. 


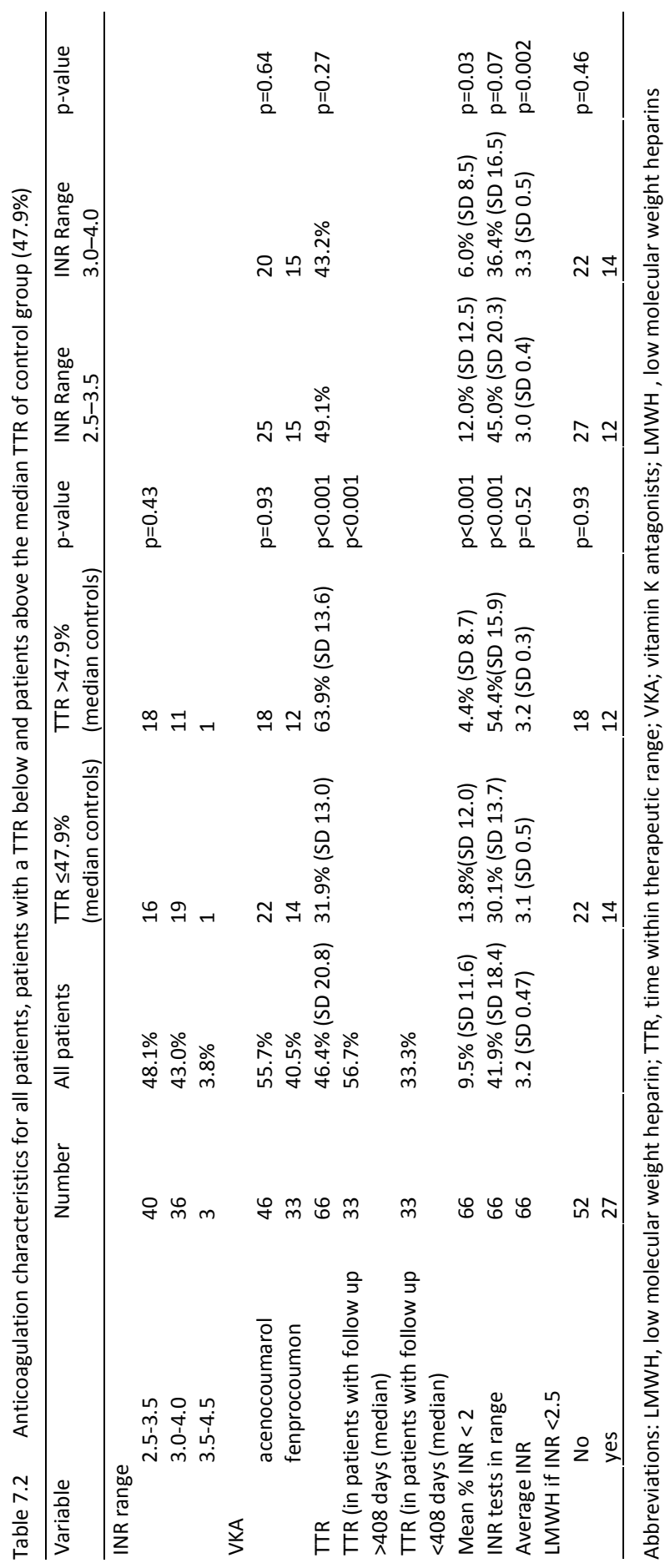


Survival analysis was performed using Kaplan Meier curves, log rank tests and Cox survival analysis. In all these analyses person time was calculated only for the patients using VKA; patients were censored at the moment they stopped with VKA use (stop anticoagulant use altogether or start of another type of antithrombotic agent (e.g. antiplatelet therapy or direct FXa inhibitors).

In Figure 7.1 and 7.2 Kaplan Meier curves are depicted for the outcome in-stent thrombosis with cut-offs defined at the median of the respective control groups (patients without in-stent thrombosis); Figure 7.1 cut-off for TTR at $\leq 47.9 \%$, and Figure 2 cut-off for proportion INR values $<2.0$ at $\leq 7.7 \%$. The Log rank tests resulted in $\mathrm{Chi}^{2}=5.54, \mathrm{p}=0.02$ and $\mathrm{Chi}^{2}=0.03, \mathrm{p}=0.86$ for figure 1 and 2 respectively.

Figure 7.1 Kaplan Meier analysis. Outcome: in-stent thrombosis for groups TTR<47.9\% (median controls) and TTR $\geq 47.9 \%$

Survival proportions: in-stent thrombosis

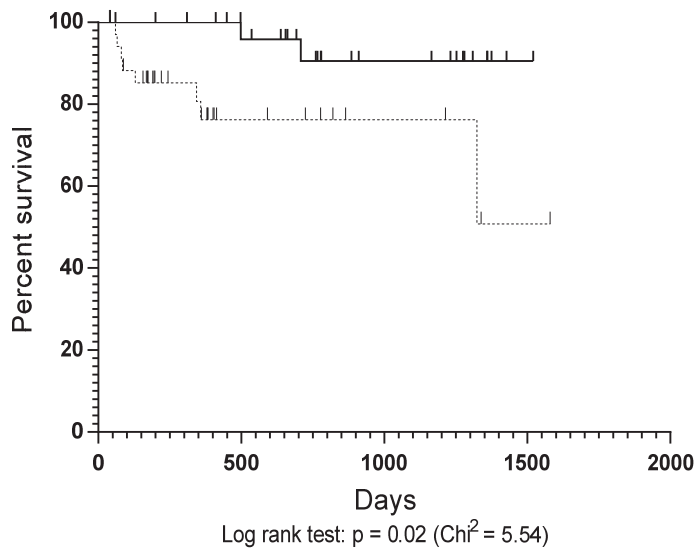

....... TTR $<47.9 \%$ (median)

$\perp$ TTR $>=47.9 \%$ (median)

Abbreviations: TTR, time within therapeutic range 
Figure 7.2 Kaplan Meier analysis. Outcome: in-stent thrombosis for groups proportion of INR $<2.0 \leq 7.7 \%$ (median controls) and proportion of INR<2.0>7.7\%

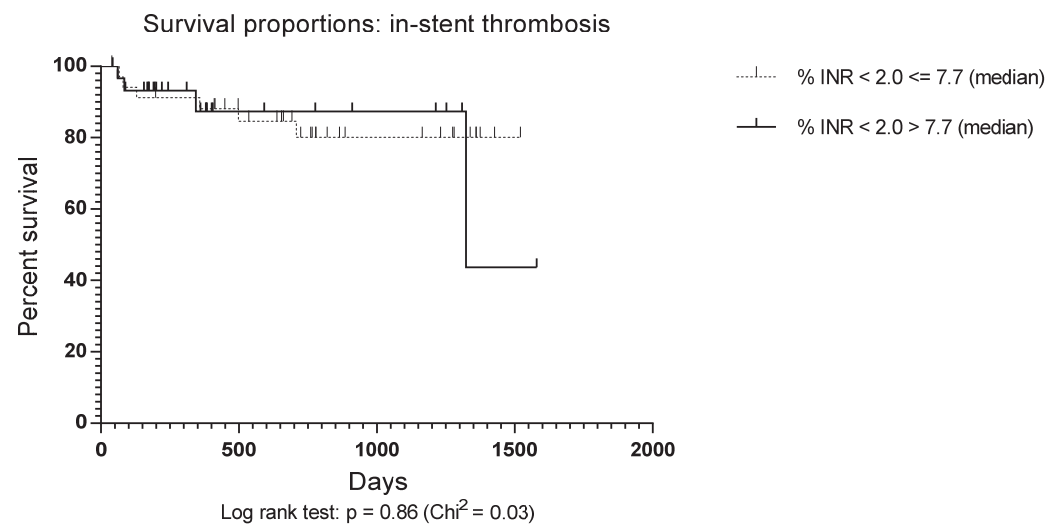

The analyses using the Cox regression are presented in Table 7.3. A linear decrease of the risk of in-stent-thrombosis associated with increasing TTR was found in all three models: the univariable, the age and sex adjusted model and in the multivariable adjusted analysis; respective HR per $1 \%$ increase in TTR were 0.954 (95\% Cl 0.920 0.988 ), 0.943 (95\% Cl 0.903-0.985) and 0.940 (95\% Cl 0.890-0.992). For INR values lower than 2.0 multivariable adjusted analysis resulted in a HR per $1 \%$ increase in proportion of 1.018 (95\% Cl $0.957-1.084)$. In the analysis of both determinants we entered the same possible confounders in our models. Pearson correlations between the independent variables TTR and proportion of INR values $<2.0$ were determined for the groups with INR ranges 2.5-3.5 and 3.0-4.0 (only PTS patients); -0.55 ( $p=0.004$ ) and $-0.27(p=0.17)$ respectively. Interactions between TTR, proportion of INR values $<2.0$ and INR target range (2.5-3.5 and 3.0-4.0) were calculated: $p=0.08$ and 0.47 respectively. To investigate the effect of the (re) initiation phase on in-stent thrombosis we calculated the TTR and proportion of INR values $<2.0$ for the first 8 weeks post-surgery. Overall non-significant results were found; in models using the same confounder set as described earlier the increase of $1 \%$ in TTR resulted in a HR of 1.023 (95\% Cl 0.980-1.069); an increase of $1 \%$ in the proportion of INR values < 2.0 resulted in a $\mathrm{HR}$ of $0.928(95 \% \mathrm{Cl} 0.848-1.015)$. The proportional hazard assumptions were not violated for TTR and proportions of INR values $<2$; therefore the effect of these independent variables may be assumed constant over time; also for all possible confounders this assumption was not violated. 
Quality of anticoagulant therapy and in-stent thrombosis in patients with venous stents

Table 7.3 Univariable, age and sex adjusted and multivariable adjusted hazard ratios for in stent thrombosis in patients with PTS according to TTR and percentage INR<2

\begin{tabular}{llll}
\hline & $\begin{array}{l}\text { Univariable: } \\
\mathrm{HR}(95 \% \mathrm{Cl})\end{array}$ & $\begin{array}{l}\text { Age sex adjusted: } \\
\mathrm{HR}(95 \% \mathrm{Cl})\end{array}$ & $\begin{array}{l}\text { Multivariable } \\
(\mathrm{HR}(95 \% \mathrm{Cl})\end{array}$ \\
\hline TTR (per 1\% increase) & $0.954(0.920-0.988)$ & $0.943(0.903-0.985)$ & $0.940(0.890-0.992)$ \\
TTR (per 10\% increase) & $0.624(0.434-0.886)$ & $0.556(0.361-0.860)$ & $0.539(0.312-0.922)$ \\
INR <2 (per 1 \% increase & $1.024(0.969-1.083)$ & $1.033(0.975-1.096)$ & $1.018(0.957-1.084)$ \\
INR <2 (per 10\% increase & $1.268(0.730-2.220)$ & $1.384(0.776-2.501)$ & $1.195(0.664-2.240)$ \\
\hline
\end{tabular}

a Adjusted for: Age at baseline, sex, LET score, LMWH administration if INR <2.5 (yes/no), VKA: acenocoumarol or fenprocoumon. Abbreviations: HR, hazard ratio; PTS, postthrombotic syndrome; TTR, time within therapeutic range

\section{Discussion}

In this study we found that the quality of anticoagulant therapy with vitamin $\mathrm{K}$ antagonists has a strong effect on in-stent thrombosis in patients after iliofemoral stent placement. We identified TTR as the most important predictive variable that reflects the quality of anticoagulant therapy and its associated risk for in-stent thrombosis. A $10 \%$ increase in TTR resulted in a risk reduction for in-stent thrombosis of $46.1 \%$. In case of in-stent thrombosis also the proportion of INR values $<2.0$ are expected to be of interest. And although not significant, an increase of $10 \%$ in proportion of INR values < 2.0 was associated with an $18.4 \%$ increase in in-stent thrombosis. The reason for this lack of significance may be due to the relatively small sample size and the lack of contrast in the population. LMWH was prescribed for INR<2.5 in both patients without in-stent thrombosis and patients with a thrombotic event in equal proportions, which may have further obscured possible differences. In addition the overall percentage of INR values $<2.0$ was low in this population, compared to other studies. ${ }^{13-15}$

The TTR was significantly higher in patients without in-stent thrombosis and remained an independent predictor of thrombotic complications after correction for possible confounders. Interestingly, the proportion INR $<2.0$ between the groups with TTR below and above median of controls differs considerably (13.8\% (SD 12.0) versus $4.4 \%$ (SD 8.7) and is highly significant, indicating that the effect of TTR can be explained at least partially by the fact that a low TTR results in increased proportions of sub therapeutic INR values. Furthermore, strong correlations between TTR and proportion of INR values <2.0 were observed, especially in the group with INR range 2.5-3.5. Besides the association between TTR and sub therapeutic INR values, another phenomenon with regard to TTR and in-stent thrombosis might play a role. Patients with low TTR experience more periods of increasing/decreasing effects of VKA; during 
periods of increasing effects the relatively low concentrations of active procoagulants protein $\mathrm{C}$ and protein $\mathrm{S}$ compared to high concentrations of active procoagulant proteins FX and prothrombin might induce a prothrombotic state irrespective of the INR value.

Compared to other studies low rates of TTR were found in our, overall young study population. ${ }^{13}$ This can be explained at least partially by the fact that the study population consists of patients with a relatively short treatment period over which TTR was calculated. This, combined with the fact that in patients with shorter followup lower TTR values are recorded because (re) initiation after surgery of VKA therapy, leads to unstable INR values during the first 6 weeks and results in low TTR. ${ }^{21}$

The sample size of our study was small. This can be explained by the fact that venous stenting is a relatively new treatment modality, and is not yet performed on a large scale. There are however also several strengths of our study, including the prospective design, the clear definition of endpoints, an objectively documented recurrence, and regular follow up of patients.

Based on the results of our study we conclude that following a stenting procedure in patients with PTS, the quality of anticoagulant treatment is of paramount importance. The fact that an overall low average TTR was recorded in this specific peri-procedural setting may indicate the need for stricter monitoring of anticoagulant treatment or for alternative treatment modalities. 


\section{References}

1. Kahn SR, Shrier I, Julian JA, Ducruet T, Arsenault L, Miron MJ, Roussin A, Desmarais S, Joyal F, Kassis J, Solymoss S, Desjardins L, Lamping DL, Johri M, Ginsberg JS. Determinants and time course of the postthrombotic syndrome after acute deep venous thrombosis. Ann Intern Med. 2008;149:698-707.

2. AbuRahma AF, Perkins SE, Wulu JT, Ng HK. Iliofemoral deep vein thrombosis: conventional therapy versus lysis and percutaneous transluminal angioplasty and stenting. Ann Surg. 2001;233:752-60.

3. Titus JM, Moise MA, Bena J, Lyden SP, Clair DG. Iliofemoral stenting for venous occlusive disease. J Vasc Surg. 2011;53:706-12.

4. Sillesen $\mathrm{H}$, Just $\mathrm{S}$, Jorgensen $\mathrm{M}$, Baekgaard $\mathrm{N}$. Catheter directed thrombolysis for treatment of iliofemoral deep venous thrombosis is durable, preserves venous valve function and may prevent chronic venous insufficiency. Eur J Vasc Endovasc Surg. 2005;30:556-62.

5. Kolbel T, Lindh M, Holst J, Uher P, Eriksson KF, Sonesson B, Malina M, Ivancev K. Extensive acute deep vein thrombosis of the iliocaval segment: midterm results of thrombolysis and stent placement. J Vasc Interv Radiol. 2007;18:243-50.

6. Baekgaard N, Broholm R, Just S, Jorgensen M, Jensen LP. Long-term results using catheter-directed thrombolysis in 103 lower limbs with acute iliofemoral venous thrombosis. Eur J Vasc Endovasc Surg. 2010;39:112-7.

7. Manninen $\mathrm{H}$, Juutilainen $\mathrm{A}$, Kaukanen $\mathrm{E}$, Lehto $\mathrm{S}$. Catheter-directed thrombolysis of proximal lower extremity deep vein thrombosis: a prospective trial with venographic and clinical follow-up. Eur J Radiol. 2012;81:1197-202.

8. Sharifi M, Mehdipour M, Bay C, Smith G, Sharifi J. Endovenous therapy for deep venous thrombosis: the TORPEDO trial. Catheter Cardiovasc Interv. 2010;76:316-25.

9. Kim JY, Choi D, Guk Ko Y, Park S, Jang Y, Lee do Y. Percutaneous treatment of deep vein thrombosis in May-Thurner syndrome. Cardiovasc Intervent Radiol. 2006;29:571-5.

10. Eijgenraam $\mathrm{P}$, ten Cate $\mathrm{H}$, ten Cate-Hoek AJ. Venous stenting after deep venous thrombosis and antithrombotic therapy: a systematic review. Rev Vasc Med. 2014;2:88-97.

11. Douketis JD, Spyropoulos AC, Spencer FA, Mayr M, Jaffer AK, Eckman MH, Dunn AS, Kunz R. Perioperative management of antithrombotic therapy: Antithrombotic Therapy and Prevention of Thrombosis, 9th ed: American College of Chest Physicians Evidence-Based Clinical Practice Guidelines. Chest. 2012;141:e326S-50S.

12. Palareti G, Legnani C, Cosmi B, Guazzaloca G, Cini M, Mattarozzi S. Poor anticoagulation quality in the first 3 months after unprovoked venous thromboembolism is a risk factor for long-term recurrence. J Thromb Haemost. 2005;3:955-61.

13. Mearns ES, Kohn CG, Song JS, Hawthorne J, Meng J, White CM, Raut MK, Schein JR, Coleman CI. Metaanalysis to assess the quality of international normalized ratio control and associated outcomes in venous thromboembolism patients. Thromb Res. 2014;134:310-9.

14. van Dongen CJ, Prandoni P, Frulla M, Marchiori A, Prins $\mathrm{MH}$, Hutten BA. Relation between quality of anticoagulant treatment and the development of the postthrombotic syndrome. J Thromb Haemost. 2005;3:939-42.

15. Chitsike RS, Rodger MA, Kovacs MJ, Betancourt MT, Wells PS, Anderson DR, Chagnon I, G LEG, Solymoss S, Crowther MA, Perrier A, White RH, Vickars LM, Ramsay T, Kahn SR. Risk of postthrombotic syndrome after subtherapeutic warfarin anticoagulation for a first unprovoked deep vein thrombosis: results from the REVERSE study. J Thromb Haemost. 2012;10:2039-44.

16. Strijkers RH, Arnoldussen CW, Wittens CH. Validation of the LET classification. Phlebology. 2015; 30:14-9.

17. Arnoldussen $\mathrm{CW}$, Toonder I, Wittens $\mathrm{CH}$. A novel scoring system for lower-extremity venous pathology analysed using magnetic resonance venography and duplex ultrasound. Phlebology. 2012;27 Suppl 1:163-70.

18. Neglen P, Hollis KC, Olivier J, Raju S. Stenting of the venous outflow in chronic venous disease: longterm stent-related outcome, clinical, and hemodynamic result. J Vasc Surg. 2007;46:979-90. 


\section{Chapter 7}

19. de Graaf R, de Wolf M, Sailer AM, van Laanen J, Wittens C, Jalaie H. Iliocaval confluence stenting for chronic venous obstructions. Cardiovasc Intervent Radiol. 2015;38:1198-204.

20. Rosendaal FR, Cannegieter SC, van der Meer FJ, Briet E. A method to determine the optimal intensity of oral anticoagulant therapy. Thromb Haemost. 1993;69:236-9.

21. Gadisseur AP, van der Meer FJ, Adriaansen HJ, Fihn SD, Rosendaal FR. Therapeutic quality control of oral anticoagulant therapy comparing the short-acting acenocoumarol and the long-acting phenprocoumon. Br J Haematol. 2002;117:940-6. 


\section{Chapter 8}

General discussion 
Chapter 8 
In this chapter, the main findings of this thesis will be discussed. Three topics are discussed: first, a study on clinical decision support (CDS) with regard to thromboprophylaxis in non-surgical hospital patients; second, safety and efficacy issues of bridging anticoagulation and finally, safety and efficacy issues of anticoagulation after venous stenting. All safety issues concern the risk of minor or major bleedings; efficacy aspects involve the risk of thromboembolism (TE), both venous and arterial.

\section{Summary}

In Chapter $\mathbf{2}$ practical aspects of thromboprophylaxis in hospitalized medical patients were assessed. The adherence to guidelines was measured before and after introduction of a CDS system. Contrary to expectations from literature no improvement was observed in guideline adherence towards antithrombotic prophylaxis.

In Chapter $\mathbf{3}$ a systematic literature review is presented, evaluating bridging therapy compared to vitamin $\mathrm{K}$ antagonists (VKA) continuation and cessation in terms of bleeding and TE risk. The review yields 2 findings: 1) no conclusion can be drawn regarding the efficacy of bridging therapy because the low incidence of thromboembolic events; 2) bridging anticoagulation results in a higher bleeding risk compared to perioperative continuation of VKA in surgical interventions involving pacemaker/defibrillator implantations or replacements.

An observational registry assessing the practice of perioperative bridging of anticoagulation showed limited guideline adherence resulting in prolonged bridging procedures, excess treatment of patients and high bleeding rates (Chapter 4).

An explorative study assessing the mechanisms underlying bridging therapy showed that whereas cessation of VKA normalized the INR value at the day of surgery, in a number of individuals clinically relevant residual anti-Xa activity was measured on the day of the intervention. In spite of this, we did not find a clear-cut association between residual anti-Xa activity and post interventional bleeding in this small series of patients. A detailed analysis of coagulation proteins yielded a pattern of procoagulant effects involving 3 distinct elements: a marked effect of FXI in the thrombin generation assay, a reduced activity of the APC pathway and a postoperative rise in FVIII and fibrinogen (Chapter 5).

A systematic review of studies evaluating the effectiveness of venous stenting in relation to postthrombotic complaints exposed an apparent lack of concern for antithrombotic therapy in relation to the stenting procedures, resulting in a marked 
variation in anticoagulant management strategies, both in duration and intensity. (Chapter 6)

To investigate the importance of anticoagulation in relation to in-stent thrombosis after venous stenting a study was performed in patients undergoing venous stenting. When analyzing time in the therapeutic range (TTR) in patients treated with VKA, we observed that higher TTR, a measure of quality of anticoagulation, offers better protection against in-stent thrombosis (Chapter 7).

\section{Clinical decision support for thromboprophylaxis}

\section{Findings in perspective}

Deep venous thrombosis (DVT) and pulmonary embolism (PE), collectively referred to as venous thromboembolism (VTE), is an underestimated health problem. Different studies demonstrate that in only $30-50 \%$ of the patients indicated for prophylaxis appropriate measures are indeed taken. ${ }^{1-3}$ Thromboprophylaxis is underused in dayto-day practice, while LMWH has proven to be very effective in the prevention of VTE in surgical and non-surgical patients. ${ }^{4-6} \mathrm{CDS}$, varying from simple electronic alerts to extensive fill-in lists of (weighed) risk factors, integrated in digital patient's records, are tools that may potentially improve guideline adherence and reduce the risk of inhospital VTE. We found no improvement in guideline adherence towards antithrombotic prophylaxis in medical patients after the introduction of CDS in our pilot study, performed in the Maastricht University Medical Center. Guidelines were followed in $59.4 \%$ both before and after the introduction of CDS. There was however a non-significant shift towards over-treatment, which may be indicative of higher prophylaxis awareness. The finding that CDS did not result in higher guideline adherence is not coherent with results presented in other studies. The introduction of CDS is in several studies associated with increased rates of per protocol administration of VTE prophylaxis, increased rates of administration of VTE prophylaxis in general ${ }^{7}$ and even with reduced rates of VTE. ${ }^{8}$ The observed lack of improvement in adherence in this pilot study could, at least partially, be caused by the suboptimal use of CDS. Final use of CDS varied between $23.7 \%$ and $57.4 \%$ for the different wards. A barrier towards implementation of CDS could have been the additional time investment needed as indicated by physicians; moreover, time consuming separate login procedures were required in order to enter CDS. Doubts whether CDS was based on solid evidence, uncertainty about the correctness of CDS advices for 'complex' patients, experienced difficulties due to the introduction of CDS and deviation from 
CDS advice caused by patient's preferences as indicated in the questionnaire presented to participating physicians, also might have contributed to the perceived lack of improvement in adherence. CDS can be a helpful tool in VTE prophylaxis management if the barriers as described above are broken down. An important condition for successful implementation is that all parties involved in patient care (physicians, ICT staff, hospital management) need to be convinced of the importance of CDS and that sufficient financial resources are available to implement a workable system.

\section{Methodological issues}

Only 128 patients participated in this pilot study and no follow-up VTE incidences were assessed. Therefore the possible association with the incidence of VTE could not be investigated. Secondly, VTE risks for patients at baseline (TO) were determined using a local protocol that did not include a risk score for bleeding. The risk assessment model (RAM) used at T1 included a calculated score for bleeding risk. This could have led to a difference in risk perception. The fact that no link was provided to the ordering system may have been the most important barrier, resulting in the limited impact of CDS in this study.

\section{Clinical implications}

The use of CDS can, if used consistently lead to improved guideline adherence and even to reduced incidence of VTE. The long-term successful effect of CDS requires training in CDS use on a regular basis to avoid a fade-out effect; this training should include an overview of the strengths of available evidence and the validation procedure of the risk scores. Also the importance of CDS in terms of avoidable VTE cases should be made clear. Only if the time investment is limited (swift log in procedures, short lists with risk factors and risk factors like age, BMI, blood results filled in automatically) a significant long-term effect of CDS can be expected. In the context of our study a questionnaire evaluating CDS use was filled in by the participating physicians: $40 \%$ had the opinion that a direct link to the ordering system would lead to an improvement; $80 \%$ questioned whether the CDS was evidence based. Bates et al. reviewed studies in which CDS systems used in different fields of expertise were evaluated and found that "speed" is a key factor for success; slow systems are ineffective.(9) A successful CDS VTE prevention system in which a direct link to the ordering system was provided was described in a study performed by Umscheid et al. ${ }^{7}$ 


\section{Future implications}

CDS supported prophylaxis is potentially superior to conventional prescription of thromboprophylaxis. In some studies CDS was effective, other studies failed to demonstrate the effect of CDS on guideline adherence. Therefore, future research should focus on the conditions needed for the implementation of an effective CDS system, such as user-friendliness and user-confidence in the correctness of the CDS generated advice.

\section{Bridging anticoagulation}

\section{Findings in perspective}

We reviewed the efficacy and safety of bridging therapy systematically. ${ }^{10}$ The most important finding of our study is that no conclusion can be drawn regarding the efficacy of bridging therapy. Overall TE incidence was low, in some studies zero. Similar findings were reported in other reviews ${ }^{11,12}$. We performed a meta-analysis assessing the risk of minor bleeding, major bleeding and overall bleeding in pacemaker/implantable defibrillator surgery. Analyses of overall bleeding resulted in a relative risk (RR) of 3.03 (95\% confidence interval $(\mathrm{Cl}), 1.86-4.95)$. A recent metaanalysis of patients undergoing pacemaker/implantable defibrillator surgery performed by Yang et al. reported that a strategy of warfarin continuation compared to bridging anticoagulation actually reduced the risk of bleeding (odds ratio (OR) 0.31 (95\% Cl, 0.18-0.53). ${ }^{13}$ The recently published BRIDGE study found in patients with atrial fibrillation undergoing overall low surgical stress inducing interventions that discontinuation of warfarin therapy was noninferior to LMWH bridging in terms of arterial TE and superior in terms of major bleeding. ${ }^{14}$ These data raise several questions that cannot be easily answered at this stage: 1 ) does the lack of advantage in the recent BRIDGE study effectively rule out application of bridging in all surgical procedures (including high thrombosis risk procedures and high risk patients)? 2) is LMWH effective, but are we over-dosing, given the residual anti-Xa levels on the day of the intervention and the apparent and in several analyses observed elevated bleeding risk? Or is bridging started to close to surgery? In an exploratory study on bridging anticoagulation we tried to get a better insight into the different mechanisms operational around the intervention. Thus, in a small series of patients we were able to collect blood samples over 9 consecutive days and found that on the day of the intervention LMWH associated anti-Xa levels $>0.05 \mathrm{IU} / \mathrm{ml}$, were still detectable in $84.6 \%$ of the population. Possible contributors to this residual anticoagulant activity 
were reduced renal clearance and $\mathrm{BMI}>30 \mathrm{~kg} / \mathrm{m}^{2}$.(15) Based on our study one may speculate that the timeframe of 24 hours between the last dose of LMWH and surgery should be extended. In a study performed by Douketis et al. residual anti-Xa effects of $>0.10 \mathrm{IU} / \mathrm{ml}$ just before surgery were observed in $16.4 \%$ of the population ${ }^{16}$ This residual anti-Xa effect might play a contributory role in postoperative bleeding; a systematic review showed that patients bridged with therapeutic doses of LMWH had a significantly increased risk of bleeding compared to patients bridged with prophylactic/intermediate doses of $\mathrm{LMWH}^{17}$ These data show the uncertainty that pertains to both the time interval of bridging to surgery and the actual doses that should be employed. Moreover, there is uncertainty as to which LMWH preparations should be used for bridging for which indication. For instance, for bridging patients with mechanical heart valves there are only few bridging studies done with enoxaparin, ${ }^{18,19}$ while in practice all kinds of LMWH preparations are used. One way to reduce uncertainty might be to monitor LMWH activity around surgery, by anti-Xa activity measurements. Optimal anti-Xa levels are unknown for this indication, hence, laboratory based optimization is in fact impossible. Overall, one should conclude that after all, there is no firm basis for the use of LMWH bridging regimens, given the poor efficacy-benefit risk ratio and remarkable uncertainty about type and dose of LMWH preparation. The fact that the administration is simple, allowing ambulant administration of s.c. injections, is no sufficient argument for LMWH bridging. Thus, the application of LMWH bridging, if still warranted, such as in patients with mechanical heart valves undergoing surgery, should be carefully re-considered.

Unfortunately and probably also driven by ease of treatment, we found that guideline adherence to bridging therapy is not optimal in a study investigating guideline adherence performed in the Netherlands. ${ }^{20}$ In fact, in about half of patients, LMWH bridging was not indicated and these data are in line with other studies. ${ }^{21,22}$ We were unable to find an association of the observed aggressive treatment with anticoagulants and the high bleeding rates, possibly due a lack of contrast in our population; in some studies post procedural therapeutic doses of LMWH were identified as a risk factor for bleeding. ${ }^{23,24}$ Possibly due to the fact that the majority of the participants were outpatients, the period of exposure to LMWH was much longer than necessary according to the ACCP guidelines; in outpatients rigidly performed INR testing is often not feasible. ${ }^{25}$ Overall TE incidence was low and in concordance with some other studies. ${ }^{24,26-28}$

In our patient series we also studied the effects of surgery on the different procoagulant mechanisms. Surgery quickly induced a marked increment in thrombin generation (TG) peak and endogenous thrombin potential (ETP) values. In a further analysis of coagulation proteins we observed that in particular FXI had significant 
effects on the rate and height of TG. This effect of FXI may support the apparent importance of this protein in driving the risk of postoperative thromboembolism, as illustrated by a recent trial showing better efficacy of FXI inhibition as compared to LMWH. ${ }^{29}$ The rise in FVIII and fibrinogen occurs gradually. Several studies report increased levels of F VIII and fibrinogen until 2 days to 72 hours postoperatively. ${ }^{30,31}$ This may in part explain the protracted risk of thrombosis following surgery. Another contributor to the thrombosis risk may be the effect of reduced sensitivity to activated protein C (APC resistance). Likely though, the level of procoagulant changes will depend on the actual surgical stress inflicted and this may explain why, in spite of the exposure to these 3 prothrombotic effects during bridging therapy, this does not immediately result in a clinically evident prothrombotic situation. In the BRIDGE trial, the vast majority of patients underwent low risk for thrombosis procedures such that the indication for bridging may have been quite low to begin with. The lesson from this study may be that the indications for bridging, as we already postulated in the above, should be revised, while for truly high risk for thrombosis patients, the question of the optimal drug and dose should also be re-established.

\section{Methodological issues}

The main limitation of our systematic literature review is the fact that most included studies are of poor methodological quality; the majority of studies have single-armed, retrospective, observational designs. Furthermore, due to different patient groups and interventions most of the meta-analyses yielded heterogeneous results; hence only 1 pooled risk estimate could be presented. Our study investigating guideline adherence had a small sample size and data were analyzed retrospectively. We were unable to compare different institutions with respect to guideline adherence, so only a local view on bridging practices could be provided. The strength of this study is the well-defined study population that allowed us to establish guideline adherence. Results of the exploratory study into biochemical mechanisms during bridging episodes was not sufficiently powered to detect possible associations between different assays and bleeding and TE.

\section{Clinical implications}

Bridging is associated with an increased risk of bleeding, possibly caused by residual anti-Xa effects on the day of the intervention. The prescribing physician should closely monitor the patient's renal function during treatment and warrant a sufficiently long interval between the last pre-operative dose of LMWH and surgery. Frequent INR checks after VKA resumption, to minimize the period in which the patient is exposed 
to both the anticoagulant effect of LMWH and VKA, might result in a decrease in postoperative bleeding risk. Physicians should be aware of the potential risks caused by over-treatment with anticoagulants during the peri-procedural period. The role of bridging anticoagulation in prevention of TE remains unclear, although several prothrombotic mechanisms caused both by the intervention and bridging seem to play a role. When the decision to apply bridging is taken, apart from exclusively weighing the risk of thromboembolism caused by patient's characteristics, as advocated by all guidelines (for instance high $\mathrm{CHADS}_{2}$ scores in combination with atrial fibrillation), the risk of thromboembolism caused by surgery should be weighed also. More invasive surgery causes an elevated post-operative TE risk, therefore LMWH bridging might still be indicated.

In the near future more often patients will be orally anticoagulated with non-vitamin $\mathrm{K}$ oral anticoagulants (NOAC). In case of interruption for surgery, bridging with LMWH should not be indicated in such patients. However, also in these patients, timing between cessation of NOAC and surgery is not self-evident. Particularly in elderly subjects with varying renal function, the apparent half-life of NOACs may be altered such that significant accumulation of NOAC activity occurs. ${ }^{32,33}$ In such cases clinically relevant bleeding risks may occur and routine check of renal (and liver) function prior to surgery is definitely warranted. In addition, the use of activity measurements like TG for NOACs may be considered, at least in patients with declining renal function.

\section{Future implications}

Sufficiently powered studies into the effects of bridging therapy on the incidence of perioperative TE should further clarify the role bridging therapy plays in combination with the prothrombotic effect of surgery in the onset of TE. Further study of the interaction of LMWH, VKA and surgery on the incidence of bleeding and TE is an important next step towards a better understanding of the mechanisms involved in bridging therapy. The possible role of the TG assay in assessing the pro or anticoagulant status of patients undergoing bridging can be explored in further studies. These future initiatives might result in more solid evidence with regard to bridging and even might result in a personalized bridging advice. 


\section{Venous stenting and antithrombotic therapy}

\section{Findings in perspective}

The main conclusions of our systematic review on venous stenting and antithrombotic therapy are: ${ }^{34} 1$ ) post procedural antithrombotic therapy is not assessed separately as a potential factor for short-term and long-term failure or success of the stenting procedure in any of the presented studies, 2) most stented patients receive warfarin only for the standard duration of the treatment of their underlying DVT. This practice is in accordance with current ACCP guidelines..$^{35}$ In only 4 studies patients received additional antiplatelet therapy after stent placement. ${ }^{36-39}$ Anticoagulants likely play a more important role than antiplatelet agents in the prevention of re-stenosis in venous stented patients with a prior DVT; ${ }^{35,40}$ in-stent thrombosis is primarily caused by increased thrombin generation. ${ }^{41}$

We identified TTR as the most important predictive variable that reflects the quality of anticoagulant therapy and its associated risk for in-stent thrombosis. A $10 \%$ increase in TTR resulted in a risk reduction for in-stent thrombosis of $46.1 \%$. In case of in-stent thrombosis also the proportion of INR values $<2.0$ are expected to be of interest. And although not significant, an increase of $10 \%$ in proportion of INR values $<2.0$ was associated with an $18.4 \%$ increase in in-stent thrombosis. The reason for this lack of significance may be due to the relatively small sample size and the lack of contrast in the population. LMWH was prescribed for INR $<2.5$ in both patients without in-stent thrombosis and patients with a thrombotic event in equal proportions, which may have further obscured possible differences. Besides the association between TTR and sub therapeutic INR values, another phenomenon with regard to TTR and in-stent thrombosis might play a role. Patients with low TTR experience more periods of increasing/decreasing effects of VKA; during periods of increasing effects the relatively low concentrations of active anticoagulants protein $C$ and protein $S$ compared to high concentrations of active procoagulant proteins FX and prothrombin might induce a prothrombotic state irrespective of the INR value.

In addition, the overall percentage of INR values $<2.0$ was low in this population, compared to other studies; ${ }^{42-44} 45.6 \%$ of patients had an INR target range of 3-4. Compared to other studies low rates of TTR were found. This can be explained at least partially by the fact that the study population consists of patients with a relatively short period in which TTR was calculated. In patients with shorter follow-up lower TTR values were recorded; (re)initiation after surgery of VKA therapy leads to unstable INR values during the first 6 weeks and results in a lower TTR. ${ }^{45}$ A very limited number of the patients in our study switched from VKA to rivaroxaban, a direct FXa inhibitor. 
Therefore, no comparison in terms of in-stent thrombosis between rivaroxaban and VKA could be made. Rivaroxaban has a more stable anticoagulant effect compared to VKA, caused by the fact that rivaroxaban shows less interactions with concomitant drug use, genetic factors and food intake. ${ }^{46}$ Possibly, rivaroxaban or other NOACs might have a better efficacy profile than VKA in patients with iliofemoral stents, but this requires new studies on this matter. The strong effect of TTR on in-stent thrombosis is an indication that further improvement of anticoagulant therapy in stented patients should at least be aimed for.

\section{Methodological issues}

A major limitation of the systematic review is the fact that no meta-analysis could be performed. Applicability of the review may be affected by the fact that most studies assessed patient groups undergoing catheter directed thrombolysis (CDT) and/or percutaneous mechanical thrombectomy (PMT) of which only a part underwent stenting; subgroups consisting of only stented patients could be assessed in only a few cases. Our study into the effect of quality of anticoagulant therapy had a small sample size. This can be explained by the fact that venous stenting is a relatively new treatment modality, not performed on a large scale. The strengths of this study are: clear definition of endpoints, objectively documented recurrence, and regular follow up of the patients.

\section{Clinical implications}

With increasing application of venous stents in patients that require anticoagulant treatment for DVT, the management of anticoagulation will deserve more consideration. Our data suggest that the quality of anticoagulant management is perhaps an even more important determinant of the risk of re-thrombosis than in patients without stents. So far, most patients with venous stents are treated with VKA, sometimes antiplatelet drugs are added to further reduce the risk of stent occlusion. The quality of VKA therapy is an important tool to avoid patency failure, and must be optimized by frequent monitoring, advice on dietary intake and emphasis of the importance of adherence. Based on results presented in different studies it is not possible to give an indication of optimal INR target range; in our study the INR target range did not influence the risk of in-stent thrombosis, therefore an INR target range of 2.0-3.0 is possibly optimal, since this range minimizes the bleeding risk. The optimal duration of anticoagulant treatment is hard to pinpoint as no time dependent effects were observed in our study. 


\section{Chapter 8}

\section{Future implications}

Future studies in which the optimal length and intensity of VKA therapy is studied may help to further improve VKA management in patients with venous stents. A comparison of conventional VKA therapy, stratified according to TTR ranges and NOAC in terms of in-stent thrombosis might also be needed to expand the range of antithrombotic options in these patients. 


\section{References}

1. Monreal M, Kakkar AK, Caprini JA, Barba R, Uresandi F, Valle R, et al. The outcome after treatment of venous thromboembolism is different in surgical and acutely ill medical patients. Findings from the RIETE registry. J Thromb Haemost. 2004;2:1892-8.

2. Goldhaber SZ, Tapson VF, Committee DFS. A prospective registry of 5,451 patients with ultrasoundconfirmed deep vein thrombosis. Am J Cardiol. 2004;93:259-62.

3. Tapson VF, Decousus H, Pini M, Chong BH, Froehlich JB, Monreal M, et al. Venous thromboembolism prophylaxis in acutely ill hospitalized medical patients: findings from the International Medical Prevention Registry on Venous Thromboembolism. Chest. 2007;132:936-45.

4. Gould MK, Garcia DA, Wren SM, Karanicolas PJ, Arcelus JI, Heit JA, et al. Prevention of VTE in nonorthopedic surgical patients: Antithrombotic Therapy and Prevention of Thrombosis, 9th ed: American College of Chest Physicians Evidence-Based Clinical Practice Guidelines. Chest. 2012;141:227-77.

5. Kahn SR, Lim W, Dunn AS, Cushman M, Dentali F, Akl EA, et al. Prevention of VTE in nonsurgical patients: Antithrombotic Therapy and Prevention of Thrombosis, 9th ed: American College of Chest Physicians Evidence-Based Clinical Practice Guidelines. Chest. 2012;141:195-226.

6. Falck-Ytter Y, Francis CW, Johanson NA, Curley C, Dahl OE, Schulman S, et al. Prevention of VTE in orthopedic surgery patients: Antithrombotic Therapy and Prevention of Thrombosis, 9th ed: American College of Chest Physicians Evidence-Based Clinical Practice Guidelines. Chest. 2012; 141:278-325.

7. Umscheid CA, Hanish A, Chittams J, Weiner MG, Hecht TE. Effectiveness of a novel and scalable clinical decision support intervention to improve venous thromboembolism prophylaxis: a quasiexperimental study. BMC Med Inform Decis Mak. 2012;12:92.

8. Maynard GA, Morris TA, Jenkins IH, Stone S, Lee J, Renvall M, et al. Optimizing prevention of hospitalacquired venous thromboembolism (VTE): prospective validation of a VTE risk assessment model. J Hosp Med. 2010;5:10-8.

9. Bates DW, Kuperman GJ, Wang S, Gandhi T, Kittler A, Volk L, et al. Ten commandments for effective clinical decision support: making the practice of evidence-based medicine a reality. Journal of the American Medical Informatics Association : JAMIA. 2003;10:523-30.

10. Eijgenraam P, Ten Cate H, Ten Cate-Hoek AJ. Safety and Efficacy of Bridging with Low Molecular Weight Heparins: A Systematic Review and Partial Meta-Analysis. Curr Pharm Des. 2013;19:4014-23.

11. Jamula E, Douketis JD, Schulman S. Perioperative anticoagulation in patients having implantation of a cardiac pacemaker or defibrillator: a systematic review and practical management guide. J Thromb Haemost. 2008;6:1615-21.

12. Levi M, Eerenberg E, Kamphuisen PW. Periprocedural reversal and bridging of anticoagulant treatment. Neth J Med. 2011;69:268-73.

13. Yang $X$, Wang Z, Zhang $Y$, Yin X, Hou Y. The safety and efficacy of antithrombotic therapy in patients undergoing cardiac rhythm device implantation: a meta-analysis. Europace. 2015.

14. Douketis JD, Spyropoulos AC, Kaatz S, Becker RC, Caprini JA, Dunn AS, et al. Perioperative bridging anticoagulation in patients with atrial fibrillation. N Engl J Med. 2015;373:823-33.

15. Clark NP. Low-molecular-weight heparin use in the obese, elderly, and in renal insufficiency. Thromb Res. 2008;123:58-61.

16. Douketis JD, Woods K, Foster GA, Crowther MA. Bridging anticoagulation with low-molecular-weight heparin after interruption of warfarin therapy is associated with a residual anticoagulant effect prior to surgery. Thromb Haemost. 2005;94:528-31.

17. Siegal D, Yudin J, Kaatz S, Douketis JD, Lim W, Spyropoulos AC. Periprocedural heparin bridging in patients receiving vitamin $\mathrm{K}$ antagonists: systematic review and meta-analysis of bleeding and thromboembolic rates. Circulation. 2012;126:1630-9.

18. Won KB, Lee SH, Chang HJ, Shim CY, Hong GR, Ha JW, et al. Safety and cost-effectiveness of bridge therapies for invasive dental procedures in patients with mechanical heart valves. Yonsei Med J. 2014;55:937-43. 
19. Hammerstingl C, Tripp C, Schmidt H, von der Recke G, Omran H. Periprocedural bridging therapy with low-molecular-weight heparin in chronically anticoagulated patients with prosthetic mechanical heart valves: experience in 116 patients from the prospective BRAVE registry. J Heart Valve Dis. 2007;16: 285-92.

20. Eijgenraam $\mathrm{P}$, ten Cate $\mathrm{H}$, ten Cate-Hoek AJ. Practice of bridging anticoagulation: guideline adherence and risk factors for bleeding. Neth J Med. 2014;72:157-64.

21. Krane LS, Laungani R, Satyanarayana R, Kaul S, Bhandari M, Peabody JO, et al. Robotic-assisted radical prostatectomy in patients receiving chronic anticoagulation therapy: role of perioperative bridging. Urology. 2008;72:1351-5.

22. Skolarus LE, Morgenstern LB, Froehlich JB, Lisabeth LD, Brown DL. Guideline-discordant periprocedural interruptions in warfarin therapy. Circ Cardiovasc Qual Outcomes. 2011;4:206-10.

23. Robinson M, Healey JS, Eikelboom J, Schulman S, Morillo CA, Nair GM, et al. Postoperative lowmolecular-weight heparin bridging is associated with an increase in wound hematoma following surgery for pacemakers and implantable defibrillators. Pacing Clin Electrophysiol. 2009;32:378-82.

24. Jaffer AK, Brotman DJ, Bash LD, Mahmood SK, Lott B, White RH. Variations in perioperative warfarin management: outcomes and practice patterns at nine hospitals. Am J Med. 2010;123:141-50.

25. Deerhake JP, Merz JC, Cooper JV, Eagle KA, Fay WP. The duration of anticoagulation bridging therapy in clinical practice may significantly exceed that observed in clinical trials. J Thromb Thrombolysis. 2007;23:107-13.

26. Pengo V, Cucchini U, Denas G, Erba N, Guazzaloca G, La Rosa L, et al. Standardized low-molecularweight heparin bridging regimen in outpatients on oral anticoagulants undergoing invasive procedure or surgery: an inception cohort management study. Circulation. 2009;119:2920-7.

27. Hammerstingl C, Omran H. Bridging of oral anticoagulation with low-molecular-weight heparin: experience in 373 patients with renal insufficiency undergoing invasive procedures. Thromb Haemost. 2009;101:1085-90.

28. Malato A, Saccullo G, Lo Coco L, Caramazza D, Abbene I, Pizzo G, et al. Patients requiring interruption of long-term oral anticoagulant therapy: the use of fixed sub-therapeutic doses of low-molecularweight heparin. J Thromb Haemost. 2010;8:107-13.

29. Buller HR, Bethune C, Bhanot S, Gailani D, Monia BP, Raskob GE, et al. Factor XI antisense oligonucleotide for prevention of venous thrombosis. N Engl J Med. 2015;372:232-40.

30. Oberweis BS, Cuff G, Rosenberg A, Pardo L, Nardi MA, Guo Y, et al. Platelet aggregation and coagulation factors in orthopedic surgery. J Thromb Thrombolysis. 2014;38;430-8.

31. Nygaard OP, Unneberg K, Reikeras O, Osterud B. Thromboplastin activity of blood monocytes after total hip replacement. Scandinavian journal of clinical and laboratory investigation. 1990;50:183-6.

32. Harper P, Young L, Merriman E. Bleeding risk with dabigatran in the frail elderly. N Engl J Med. 2012;366:864-6.

33. Wychowski MK, Kouides PA. Dabigatran-induced gastrointestinal bleeding in an elderly patient with moderate renal impairment. Ann Pharmacother. 2012;46:10.

34. Eijgenraam $P$, ten Cate $H$, ten Cate-Hoek AJ. Venous stenting after deep venous thrombosis and antithrombotic therapy: A systematic review. Rev Vacr Med. 2014;2:88-97.

35. Kearon C, Akl EA, Comerota AJ, Prandoni P, Bounameaux H, Goldhaber SZ, et al. Antithrombotic therapy for VTE disease: Antithrombotic Therapy and Prevention of Thrombosis, 9th ed: American College of Chest Physicians Evidence-Based Clinical Practice Guidelines. Chest. 2012;141:419S-94.

36. Nayak L, Hildebolt CF, Vedantham S. Postthrombotic syndrome: feasibility of a strategy of imagingguided endovascular intervention. J Vasc Interv Radiol. 2012;23:1165-73.

37. Raju S, Neglen P. Percutaneous recanalization of total occlusions of the iliac vein. J Vasc Surg. 2009;50:360-8.

38. Sharifi M, Mehdipour M, Bay C, Smith G, Sharifi J. Endovenous therapy for deep venous thrombosis: the TORPEDO trial. Catheter Cardiovasc Interv. 2010;76:316-25.

39. Wahlgren $\mathrm{CM}$, Wahlberg E, Olofsson P. Endovascular treatment in postthrombotic syndrome. Vasc Endovascular Surg. 2010;44:356-60.

40. Meissner $\mathrm{MH}$. Indications for platelet aggregation inhibitors after venous stents. Phlebology. 2013; 28:91-8. 
41. Geerts WH, Pineo GF, Heit JA, Bergqvist D, Lassen MR, Colwell CW, et al. Prevention of venous thromboembolism: the Seventh ACCP Conference on Antithrombotic and Thrombolytic Therapy. Chest. 2004;126:338-400.

42. Mearns ES, Kohn CG, Song JS, Hawthorne J, Meng J, White CM, et al. Meta-analysis to assess the quality of international normalized ratio control and associated outcomes in venous thromboembolism patients. Thromb Res. 2014;134:310-9.

43. van Dongen $\mathrm{CJ}$, Prandoni P, Frulla M, Marchiori A, Prins MH, Hutten BA. Relation between quality of anticoagulant treatment and the development of the postthrombotic syndrome. J Thromb Haemost. 2005;3:939-42.

44. Chitsike RS, Rodger MA, Kovacs MJ, Betancourt MT, Wells PS, Anderson DR, et al. Risk of postthrombotic syndrome after subtherapeutic warfarin anticoagulation for a first unprovoked deep vein thrombosis: results from the REVERSE study. J Thromb Haemost. 2012;10:2039-44.

45. Gadisseur AP, van der Meer FJ, Adriaansen HJ, Fihn SD, Rosendaal FR. Therapeutic quality control of oral anticoagulant therapy comparing the short-acting acenocoumarol and the long-acting phenprocoumon. Br J Haematol. 2002;117:940-6.

46. Ageno W, Gallus AS, Wittkowsky A, Crowther M, Hylek EM, Palareti G, et al. Oral anticoagulant therapy: Antithrombotic Therapy and Prevention of Thrombosis, 9th ed: American College of Chest Physicians Evidence-Based Clinical Practice Guidelines. Chest. 2012;141:44-88. 
Samenvatting 


\section{Studies naar veiligheidsaspecten van verschillende antistollingsbehandelingen}

Bij de behandeling van patiënten met antistollingsmiddelen zoals laag moleculairgewicht heparine (LMWH) en/of vitamine $\mathrm{K}$ antagonisten (VKA) dient te allen tijde een afweging gemaakt te worden tussen het risico op een trombo-embolie (TE) en het risico op een bloeding (mede) ten gevolge van de behandeling met deze preparaten. De balans tussen deze risico's wordt onder andere bepaald door de karakteristieken van de patiënt, de aard en ernst van de aandoening waarvoor antistolling wordt gegeven en of er een ingreep gaat plaatsvinden. In richtlijnen is getracht het beschikbare bewijs samen te vatten tot bruikbare richtsnoeren voor de behandeling van patiënten.

Het eerste deel van dit proefschrift behandelt de invoer en evaluatie van een clinical decision system (CDS) dat een gepersonaliseerd behandeladvies met LMWH ter voorkoming van veneuze trombo-embolie (VTE) genereert voor patiënten die op een afdeling interne geneeskunde opgenomen zijn. Eerdere studies wijzen uit dat deze patiënten vaak geen profylaxe ontvangen terwijl de indicatie hiervoor wel aanwezig is. Uit onderzoek blijkt eveneens dat de juiste toepassing van CDS de incidentie van diep veneuze trombose en longembolieën kan doen afnemen. In Hoofdstuk $\mathbf{2}$ wordt deze studie beschreven. Een in het elektronisch patiëntendossier geïntegreerde risicoscore werd gebruikt om het risico op een veneuze trombo-embolie (VTE) en het bloedingsrisico te schatten. We concluderen dat er geen verbetering heeft plaatsgevonden in de naleving van richtlijnen met betrekking tot tromboseprofylaxe na introductie van CDS; er was een niet-significante toename van overbehandeling met LMWH waarneembaar. Mogelijke verklaringen voor deze resultaten zijn suboptimaal gebruik van CDS, afwijking van CDS ten gevolge van voorkeuren van de patiënt en een verhoogde waakzaamheid voor VTE risico door invoering CDS.

Het tweede deel van dit proefschrift behandelt overbruggingstherapie: de vervanging van VKA door LMWH in de periode rondom een operatie. Richtlijnen voor deze aanpak worden niet gedragen door veel bewijs; mede om deze reden presenteren we in hoofdstuk 3 de resultaten van een 'systematic review' van de beschikbare literatuur over dit onderwerp. Overbruggingstherapie wordt vergeleken met de perioperatieve continuering van VKA en met het perioperatief stoppen van VKA. We hebben als uitkomsten postoperatieve bloeding en TE gedefinieerd. Op grond van de resultaten van deze review valt geen conclusie te trekken over het effect van overbrugging op TE, vergeleken met de overige 2 behandelopties; de incidentie van TE 
is in alle studies laag. Uit een meta-analyse uitgevoerd met behulp van studies waarin patiënten pacemaker/implanteerbare defibrillator chirurgie ondergingen bleek overbruggingstherapie vergeleken met VKA continuering een verhoogd risico op postoperatieve bloedingen te veroorzaken.

In hoofdstuk 4 wordt beoordeeld in hoeverre de richtlijnen voor overbruggingstherapie zoals die zijn uitgegeven door het American College of Chest Physicians (ACCP) zijn gevolgd bij patiënten die zijn overbrugd met LMWH en zijn opgenomen in het Maastricht University Medical Center (MUMC+); daarnaast hebben we mogelijke risicofactoren voor bloedingen vastgesteld. Overbruggingsrichtlijnen werden slecht opgevolgd, wat leidde tot langdurige behandelingen, overbehandeling en een hoge incidentie van bloedingen. De meerderheid van de patiënten had een laag TE risicoprofiel en onderging ingrepen met een laag risico op bloedingen. We hebben, in tegenstelling tot andere auteurs, in deze studie geen verband kunnen leggen tussen deze agressieve behandeling en het veelvuldig optreden van bloedingen. De lage incidentie van TE is in overeenstemming met andere studies. Bij patiënten met een verminderde creatinineklaring dienen verlaagde doses $\mathrm{LMWH}$ overwogen te worden, ten einde het bloedingsrisico te minimaliseren.

In hoofdstuk 5 hebben we de effecten van overbruggingstherapie beoordeeld aan de hand van verschillende laboratoriumbepalingen, waaronder INR, anti-Xa, concentraties van verschillende stollingsfactoren en trombinegeneratie (TG). Een aantal patiënten werd gedurende de gehele overbruggingsperiode van negen dagen gevolgd door middel van dagelijkse bloedafnames tijdens een pilot studie. Ondanks residuele anti-Xa effecten is er een opmerkelijke verhoging van TG vastgesteld in relatie tot de ingreep. Perioperatief treden er drie pro-trombotische effecten op: FXI afhankelijke TG, afgenomen activiteit van de geactiveerde proteïne $C$ route en de postoperatieve toename van acuut fase eiwitten FVIII en fibrinogeen. Verder onderzoek is noodzakelijk om de rol van verschillende assays tijdens de overbruggingsperiode vast te stellen.

De laatste twee decennia heeft het plaatsen van veneuze stents na een diep veneuze trombose (DVT) meer aandacht gekregen. Het laatste deel van het proefschrift behandelt antistolling na veneuze stenting. Het merendeel van de studies dat de veiligheid en effectiviteit van deze ingreep evalueert is van matige methodologische kwaliteit. In hoofdstuk 6 worden de resultaten van een 'systematic review' gepresenteerd. In deze review wordt getracht de beschikbare literatuur over veneuze stentplaatsing en antitrombotische therapie te ordenen. Antitrombotische therapie 
lijkt op basis van de beschikbare literatuur geen van de uitkomsten (terugkerende DVT, doorgankelijkheid van de stent, post trombotisch syndroom of bloedingen) te beïnvloeden. Dit kan worden verklaard omdat tot op heden is de associatie tussen de kwaliteit van antistollingstherapie met VKA, uitgedrukt als de proportie van de tijd die de patiënt zich in de juiste therapeutische range (TTR) bevindt en de effectiviteit van stentplaatsing nog niet expliciet is bestudeerd. In hoofdstuk 7 wordt de relatie tussen kwaliteit van antistolling en in-stent trombose onderzocht. We concluderen dat een verhoging van de TTR bescherming biedt tegen in-stent trombose. De proportie van INR waarden $<2.0$ komt uit de analyses niet als risicofactor voor in-stent trombose. 
Valorisatie 
In westerse landen wordt op grote schaal, in het bijzonder bij ouderen, gebruik gemaakt van antistollingsmiddelen zoals vitamine $K$ antagonisten (VKA) en laag moleculairgewicht heparine (LMWH). Indicaties voor het gebruik van deze middelen zijn onder meer atrium fibrilleren, kunstmatige hartklep, diep veneuze trombose (DVT) (therapeutisch en profylactisch), veneuze stentplaatsing en overbrugging bij ingrepen. De effectiviteits- en veiligheidsaspecten van behandeling met VKA en/of LMWH betreffen hoofdzakelijk het risico op bloedingen en trombo-embolieën (TE) ten gevolge van de behandeling met deze middelen en de aandoening waarvoor de antistolling wordt voorgeschreven. Er zijn in dit proefschrift drie onderwerpen behandeld.

Het eerste deel (Hoofdstuk 2) behandelt de introductie en de evaluatie van het introduceren van een clinical decision support (CDS) waarbij een geautomatiseerd en gepersonaliseerd behandeladvies met LMWH ter voorkoming van veneuze tromboembolie (VTE) wordt gegenereerd. Studies tonen aan dat een belangrijk deel van de patiëntenpopulatie met een indicatie voor profylactisch LMWH gebruik dit niet ontvangt; dit geldt in het bijzonder voor niet chirurgische patiënten.(1) Eerdere, op verschillende locaties ingevoerde CDS systemen leidden tot een afname van de incidentie van DVT.(2) De relevantie van de door ons uitgevoerde evaluatie van CDS in het Maastricht University Medical Center (MUMC+), is gelegen in de constatering dat er aan een succesvolle invoering van CDS een aantal voorwaarden zijn gekoppeld waaraan moet worden voldaan. De belangrijkste voorwaarden zijn: minimale tijdsinvestering door voorschrijvend artsen, goede voorlichting aan gebruikers over de mogelijke positieve effecten van CDS in termen van VTE incidentie en de wetenschappelijke basis van het gegenereerde advies. Hiernaast lijkt het van groot belang dat de invoer 'breed wordt gedragen' door zowel directie, ICT-diensten als artsen. Deze constateringen kunnen beleidsmakers en artsen die betrokken zijn bij de totstandkoming van toekomstige CDS projecten helpen de kans op een effectief CDS te vergroten. De investering in CDS kan eenvoudig worden terugverdiend doordat minder kosten hoeven te worden gemaakt voor de behandeling van DVT en longembolieën. Longembolieën leiden niet zelden tot het overlijden van de patiënt, terwijl DVT kan leiden tot levenslange beperkingen bij de patiënt.

Deel twee van dit proefschrift (Hoofdstuk 3-5) behandelt overbruggingstherapie: de vervanging van VKA door LMWH in de periode rondom een operatie. Deze aanpak wordt wereldwijd toegepast. Richtlijnen voor deze aanpak worden niet gedragen door uitvoerig bewijs.(3) De huidige, in richtlijnen aanbevolen manier om overbruggingstherapie uit te voeren leidt tot een verhoogd bloedingsrisico, terwijl het 
TE risico onduidelijk blijft.(4) Monitoring van de hemostase bij de overbrugging van antistolling bestaat uit het uitvoeren van INR bepalingen. Als de INR zich op de dag van de ingreep rond de 1 bevindt, kan de ingreep doorgang vinden; als na de ingreep de INR twee opeenvolgende dagen 2.0 of hoger is kan er worden gestopt met LMWH toediening. Uit de in dit proefschrift gepresenteerde resultaten blijkt dat deze aanpak wellicht tekort schiet; een mogelijke rol voor anti-Xa bepalingen in verband met residuele antistolling ten tijde van de ingreep, in het bijzonder bij patiënten met een verminderde nierfunctie en bij patiënten die een therapeutische behandeling met LMWH ondergaan verdient zeker aandacht in verdere studies. Het gebruik van nieuwe technieken zoals de ook door ons onderzochte trombinegeneratie kan daarbij overwogen worden. Voorschrijvend artsen en trombosediensten verantwoordelijk voor de patiëntveiligheid kunnen op deze wijze het aantal postoperatieve bloedingen tijdens overbruggingsepisoden wellicht verder beperken. De door ons uitgevoerde studies kunnen een opmaat vormen voor verandering in de wijze waarop overbrugging wordt gemonitord en wellicht ook leiden tot een meer op maat afgestemde behandeling met LMWH. Een aanpassing van overbruggingsprotocollen en bevordering van bewustwording bij artsen dat bloedingsrisico's wellicht op grote schaal worden onderschat vormen een direct doel van dit proefschrift. Belangrijk is dat toekomstige onderzoekers aanvullend bewijs genereren voor de relevantie van een gewijzigde benadering van het fenomeen overbruggingstherapie.

Het laatste deel van dit proefschrift (Hoofdstuk 6 en 7) behandelt antistolling na veneuze stenting. In tot dusver gepubliceerde studies lijkt antitrombotische therapie geen van de uitkomsten (terugkerende DVT, doorgankelijkheid van de stent, post trombotisch syndroom of bloedingen) te beïnvloeden. Onze studie toont echter aan dat het risico op in-stenttrombose mede bepaald wordt door de kwaliteit van antistolling, weergegeven als de tijd binnen de therapeutische range. Artsen moeten doordrongen worden van het belang van kwaliteit van antistolling na plaatsing van een veneuze stent; dit kan betekenen dat een strikte monitoring van de INR belangrijker is dan tot dusver werd aangenomen. Wellicht is hier adequate antistolling met LMWH direct na insertie van de stent tijdelijk aangewezen. Toekomstige studies kunnen zich ook richten op de effecten van de nieuwe generatie antistollingsmiddelen, zowel de directe factor Xa als Ila remmers, die dankzij een naar verhouding stabiele farmacokinetiek wellicht sneller een stabiel niveau van antistolling kunnen realiseren. Een punt van overweging is evenwel of de vaste doseringen van deze directe remmers adequaat zijn voor alle patiënten die een veneuze stent krijgen. Specifiek onderzoek naar deze aspecten lijkt noodzakelijk. 


\section{Referenties}

1. Goldhaber SZ, Tapson VF, Committee DFS. A prospective registry of 5,451 patients with ultrasoundconfirmed deep vein thrombosis. Am J Cardiol. 2004;93:259-62.

2. Umscheid CA, Hanish A, Chittams J, Weiner MG, Hecht TE. Effectiveness of a novel and scalable clinical decision support intervention to improve venous thromboembolism prophylaxis: a quasiexperimental study. BMC medical informatics and decision making. 2012;12:92.

3. Douketis JD, Spyropoulos AC, Spencer FA, Mayr M, Jaffer AK, Eckman MH, et al. Perioperative management of antithrombotic therapy: Antithrombotic Therapy and Prevention of Thrombosis, 9th ed: American College of Chest Physicians Evidence-Based Clinical Practice Guidelines. Chest. 2012;141(2 Suppl):e326S-50S.

4. Douketis JD, Spyropoulos AC, Kaatz S, Becker RC, Caprini JA, Dunn AS, et al. Perioperative Bridging Anticoagulation in Patients with Atrial Fibrillation. N Engl J Med. 2015.

5. Eijgenraam $\mathrm{P}$, ten Cate $\mathrm{H}$, ten Cate-Hoek AJ. Venous stenting after deep venous thrombosis and antithrombotic therapy: a systematic review. Reviews in Vascular Medicine. 2014;2:88-97. 
List of publications 


\section{Publications}

1. Eijgenraam $\mathbf{P}$, Meertens $\mathbf{N}$, van den Ham R, Ten Cate $H$, Ten Cate-Hoek AJ. The effect of clinical decision support on adherence to thrombosis prophylaxis guidelines in medical patients; A single center experience. Thromb Res. 2015;135(3):464-471.

2. Eijgenraam $\mathbf{P}$, Hugo ten Cate, Arina J ten Cate-Hoek Venous stenting after deep venous thrombosis and antithrombotic therapy: a systematic review. RVM. 2014;2(3):88-97.

3. Eijgenraam $\mathbf{P}$, ten Cate $\mathrm{H}$, ten Cate-Hoek AJ. Practice of bridging anticoagulation: guideline adherence and risk factors for bleeding. Neth J Med. 2014;72(3):157164.

4. Eijgenraam $\mathbf{P}$, ten Cate $\mathrm{H}$, Ten Cate-Hoek A. Safety and efficacy of bridging with low molecular weight heparins: a systematic review and partial meta-analysis. Curr Pharm Des. 2013;19(22):4014-4023. 
Dankwoord 


\section{Dankwoord}

Promoveren is grotendeels eenzaam werk. Collega promovendi, de personen waar je het meest mee te maken hebt tijdens je werk, zijn allen bezig hun eigen stukje van de werkelijkheid te verklaren. Desalniettemin, zonder de bijdrage van een aantal mensen in totaal verschillende rollen, hadden mijn inspanningen tot niets geleid.

Allereerst dank ik mijn vrouw Jolanda en mijn kinderen Onno en Boris voor het aanhoren van mijn saaie verhalen, hun steun en liefde. Jo is degene geweest die mij heeft gemotiveerd structuur in mijn leven aan te brengen en op zoek te gaan naar een baan waarbij ik mijn kwaliteiten het best kon inzetten. Zonder jou was het allemaal niet gelukt. Jij was het die mij motiveerde op lastige momenten door te gaan met onderzoek en dit traject af te maken. Nu merk ik dat je oprecht trots bent op je vent die af en toe niet de makkelijkste is.

Mijn promotor en copromotors dienen uiteraard ook vermeld te worden. Hugo, wat ik zeer waardeer is dat je me de kans hebt gegeven te promoveren. We zijn aan dit traject begonnen zonder dat er een duidelijk plan lag. Toen de geldstroom dreigde op te drogen heb jij er voor gezorgd dat ik mijn salaris bleef ontvangen en dus verder kon met onderzoek. Je hebt moeite gedaan mij te blijven faciliteren. Ik heb sterk het vermoeden dat de 'gunfactor' hier een rol heeft gespeeld. Verder waardeer ik je brede kennis van de stolling en je gave deze op simpele wijze uit te dragen en je laagdrempeligheid. We kwamen elkaar niet dagelijks tegen, maar als ik je zag ervoer ik ons contact altijd als zeer prettig en oprecht, zonder dat we veel over elkaars persoonlijke leven te weten kwamen. Arina is eigenlijk de link met mijn bestaan als onderzoeker gebleken; door jou ben ik met Hugo in contact gekomen. Tijdens onze studie epidemiologie die we tegelijkertijd hebben doorlopen en later leerde ik je kennen als een recht-door-zee, no-nonsense mens dat zeer bevlogen is op een eerlijke manier onderzoek te doen en zeker niet vies is van hard werken. Ik herinner me dat je zelf de pen (Words) tijdens de kerstvakantie ter hand nam om mee te schrijven aan de review over veneuze stenting. Altijd beoordeelde je mijn werk kritisch en zonder dat ik lang hoefde te wachten op antwoord.

Natuurlijk ben ik Rene van den Ham van Philips Research ook dank verschuldigd. Door jouw connectie met MUMC/UM ben ik in de gelegenheid geweest te starten met mijn promotie. De Brugstudie die jij gefaciliteerd hebt heeft jammer genoeg niet de gewenste inclusie bereikt, ondanks mijn pogingen hiertoe. Tegen het einde van mijn promotie zakte de studie langzaam in door een soort moeheid van alle betrokken 
partijen. Toch waren er voldoende data beschikbaar om een hoofdstuk aan de analyse ervan te wijden en collega Henk van Ooijen in staat te stellen het 'model' van de stolling te verfijnen.

Niet onvermeld kunnen de verschillende coauteurs blijven, die altijd mijn stukken aandachtig en deskundig hebben bekeken en een belangrijke bijdrage aan dataverzameling hebben geleverd. Naast natuurlijk mijn (co)promotor(es), dank ik Nathalie Meertens, die voor onze CDS studie langdurig door SAP heeft geakkerd. Ook de bijdrage van Yvonne Henskens met haar specifieke kennis over verschillende assays heeft kwaliteit verhogend gewerkt. Tenslotte prijs ik de inbreng van zowel Mark de Wolf als verzamelaar van data van gestente patiënten en coauteur Ralph Kurstjens als kritisch lezer.

Ook bedank ik de beoordelingscommissie voor het beoordelen van mijn werk van de afgelopen 4,5 jaar.

Mijn kamergenoten, al zag ik hen slechts op de donderdagen waren allen prettig in de omgang. In de loop der jaren waren het er zes. Twee van hen wil ik niet onvermeld laten. Allereerst Minka, tevens mijn paranimf, met wie ik vaak leuke, soms melige en lachwekkende, af en toe serieuze, zelden saaie gesprekken voerde, meestal in de middag als het eind van de dag in zicht kwam. Samen slaagden we er moeiteloos in de betrekkelijkheid van ons eigen promotietraject onder woorden te brengen en verschillende collega's kritisch te evalueren (roddelen werd het nooit). Snelle Jelle was, als hij er was, duidelijk aanwezig. Zijn komst werd al aangekondigd door een krachtige, vastberaden opening van de kamerdeur. Altijd een leuke babbel!

Michael, my other 'paranimf' from Switzerland always has a positive view on science and life. He is very interested in different subjects, science is one of them. I do not know you very well, but the times we met were always nice and entertaining.

Ook de dames van de trombosedienst hebben een bijdrage geleverd aan de totstandkoming van dit geschrift. Jarenlang ontving ik trouw de lijstjes van patiënten die 'gebrigded' gingen worden van Jolanda of Loes, zodat ik vervolgens kon proberen mogelijke participanten over te halen deel te nemen aan de Brugstudie. Marieke, stille kracht en kundig bloedprikker heeft talloze bloedafnames verricht voor de Brugstudie. Toegewijd en aandachtig heeft zij belangrijk werk verricht. Carol en Nina, beide leuke dames van het lab, die soms een deel van hun weekend offerden aan de wetenschap, 
om bloed te verwerken dienen ook vermeld te worden in dit dankwoord. Rene van Oerle wil ik bedanken voor het analyseren van bloed ten behoeve van de Brugstudie.

Soms voor een praatje, soms om iets te regelen, soms voor beide bracht ik een bezoek aan de kamer van Trees en Lidewij. Deze secretaressen met ieder hun specifieke deskundigheid maakten altijd tijd me te helpen. Met Trees in het bijzonder heb ik prettige gesprekken gevoerd, dank hiervoor.

Mijn team bij Gilde opleidingen dank ik voor hun flexibele manier van omgang met mijn werktijden. Men kwam zo veel als mogelijk tegemoet aan mijn wensen; hierdoor verliepen de zaken op UM vlotter en prettiger. 
Curriculum vitae 


\section{About the author}

Pieter Eijgenraam was born on October 271963 in Leiden, the Netherlands and finished his pre-university education at the Sint Maartenscollege in Maastricht in 1984. From 1984 to 1987 he studied Dutch law at the Maastricht University (Propaedeuse 1985) and the University of Nijmegen. From 1987 to 1995 he performed several jobs. In 1995 he started the Nurse education at the Maasland ziekenhuis in Sittard and was employed in this institution until 2000. In 2000 he accepted a job as a teacher at Gilde Opleidingen in Sittard. In 2002 he finished his teacher-training course at Hogeschool Arnhem Nijmegen (HAN). In 2008 he started the master program Epidemiology at Maastricht University. His internship was carried out in Department of Epidemiology where he wrote his thesis entitled: Medical conditions (diabetes mellitus type II, peptic ulcer, hepatitis, hypertension) and pancreatic cancer. Data from the Netherlands Cohort Study (NLCS) were analyzed. The results of this thesis were published in the British Journal of Cancer (BJC). In 2011 he started a part-time PhD project at the Laboratory for Clinical Thrombosis and Haemostasis of Maastricht University, Cardiovascular Centre MUMC+. The studies performed within this project resulted in the current thesis. 
